\title{
The Velocity Dispersion Profile of the Remote Dwarf Spheroidal Galaxy Leo I: A Tidal Hit and Run?
}

\author{
Mario Mateo ${ }^{1}$, Edward W. Olszewski ${ }^{2}$, and Matthew G. Walker ${ }^{3}$
}

\begin{abstract}
We present new kinematic results for a sample of 387 stars located in and around the Milky Way satellite dwarf spheroidal galaxy Leo I. These spectra were obtained with the Hectochelle multi-object echelle spectrograph on the MMT, and cover the MgI/Mgb lines at about $5200 \AA$. Based on 297 repeat measurements of 108 stars, we estimate the mean velocity error $(1 \sigma)$ of our sample to be $2.4 \mathrm{~km} / \mathrm{s}$, with a systematic error of $\leq 1 \mathrm{~km} / \mathrm{s}$. Combined with earlier results, we identify a final sample of 328 Leo I red giant members, from which we measure a mean heliocentric radial velocity of $282.9 \pm 0.5 \mathrm{~km} / \mathrm{s}$, and a mean radial velocity dispersion of $9.2 \pm 0.4 \mathrm{~km} / \mathrm{s}$ for Leo I. The dispersion profile of Leo I is essentially flat from the center of the galaxy to beyond its classical 'tidal' radius, a result that is unaffected by contamination from field stars or binaries within our kinematic sample. We have fit the profile to a variety of equilibrium dynamical models and can strongly rule out models where mass follows light. Two-component Sersic+NFW models with tangentially anisotropic velocity distributions fit the dispersion profile well, with isotropic models ruled out at a 95\% confidence level. Within the projected radius sampled by our data $(\sim 1040 \mathrm{pc})$, the mass and V-band mass-to-light ratio of Leo I estimated from equilibrium models are in the ranges 5-7 $\times 10^{7} M_{\odot}$ and 9-14 (solar units), respectively. We find that Leo I members located outside a 'break radius' at $R_{b} \sim 400 \operatorname{arcsec}(500$ pc) exhibit significant velocity anisotropy, whereas stars interior of this radius appear consistent with an isotropic velocity distribution. We propose a heuristic model in which the break radius represents the location of the tidal radius of Leo I at perigalacticon of a highly elliptical orbit. Our scenario can account for
\end{abstract}

\footnotetext{
${ }^{1}$ Department of Astronomy, University of Michigan, 830 Dennison Building, Ann Arbor, MI 48109-1042; mmateo@umich.edu

${ }^{2}$ Steward Observatory, The University of Arizona, Tucson, AZ 85721; edo@as.arizona.edu

${ }^{3}$ Department of Astronomy, University of Michigan, 830 Dennison Building, Ann Arbor, MI 48109-1042; mgwalker@umich. edu
} 
the complex star formation history of Leo I, the presence of population segregation within the galaxy, and Leo I's large outward velocity from the Milky Way. Within the framework of our model, the lack of extended tidal arms in Leo I both perpendicular to and along the line of sight - suggests the galaxy has experienced only one perigalactic passage with the Milky Way; thus, Leo I may have been injected into its present orbit by a third body a few Gyr before perigalacticon. We discuss the plausibility of this idea within the context of hierarchical models and conclude that such an interaction is entirely possible. We also report the possible detection of a distinct kinematic structure in the Leo I field at about a $2 \sigma$ significance level.

Subject headings: galaxies: dwarf — galaxies: kinematics and dynamics — (galaxies:) Local Group — techniques: radial velocities

\section{Introduction}

An understanding of how external and internal dynamical effects combine to produce the observed kinematic properties of dwarf galaxies is fundamental to probing the nature of these systems. Since dwarfs may represent the smallest dark-matter (DM) halos that have survived to the present epoch - or simply the smallest that have retained baryons (Ferrara and Tolstoy 2000; Benson et al. 2002; Grebel and Gallagher 2004; Susa and Umemura 2004) - they represent an important link to broader structure formation models. As we learn more about the dynamical state of the Galactic halo, however, is has become clear that complex interactions of the components within the halo - including local dwarfs - may be common (Taylor and Babul 2004, 2005; Coleman et al. 2004). Detailed $n$-body models hint at the frequency of interactions (Moore et al. 1999; Kravtsov et al. 2004), while hybrid $n$ body+hydrodynamical models reveal the richness of the baryonic phenomena - episodic star formation, gas streaming - that may result (Mayer et al. 2001a,b, 2005, 2007; Kravtsov et al. 2004). Observational evidence in the halo of the Milky Way (Sagittarius: Ibata et al. 1994, Majewski et al. 2003, Belokurov et al. 2006; The Magellanic Stream: Putman et al. 1998, Connors et al. 2006; The Fornax dwarf spheroidal galaxy: Coleman and Da Costa 2004, 2005, Olszewski et al. 2006; The halo of M31: Ibata et al. 2001; Brown et al. 2006) broadly confirms model expectations that tides/encounters strongly alter the structural features of satellite dwarf systems.

To describe the halo and its interaction history fully, we need to know the nature of the DM subhalos. Nearby dwarfs and their DM halos represent the best local proxies for

the original DM seeds that built up the Milky Way (Read et al. 2006a). But to probe the 
satellites and study their DM halos in detail, we need to account for the effects of interactions, since these can strongly alter our conclusions regarding the properties of the halos (Klessen and Kroupa 1998; Fleck and Kuhn 2003; Metz et al. 2007). Moreover, understanding the tidal history of dwarfs depends on the knowledge of the total mass and structure of the DM halo of the Milky Way in which these interactions occur. The problem is deliciously interconnected and devilishly hard to solve, particularly in the absence of a secure description of the small halos comprising the Milky Way's extended halo. We have embarked on an effort to address part of this riddle by systematically studying the kinematic properties of many of satellites of the Milky Way (Walker et al. 2007a,b). The present paper focuses on the Leo I dwarf spheroidal (dSph) galaxy.

Two general cases arise when we consider the dynamical state of a galaxy such as Leo 11 . First, a galaxy can be considered to be in dynamical equilibrium if it is sufficiently isolated from outside perturbations. This case is well-understood theoretically though it does admit to various degeneracies (e.g. anisotropy and the mass distribution) and other complications (deprojections of possibly non-spherical mass or tracer distributions). Nonetheless, one can, with enough data, close in on the mass distribution from the kinematics and distribution of baryonic tracers. However, given the hierarchical nature of galaxy formation noted above, the second, more realistic expectation is that galaxies, particularly dwarf satellites, can never be in true dynamical equilibrium. The question then becomes one of degree: How far out of equilibrium is a given galaxy and how do deviations from equilibrium affect our interpretation of its kinematics?

The motions of stars in a satellite galaxy are governed by its underlying mass distribution and the external potential in which the galaxy orbits. To complicate matters, the external potential may be asymmetric, or significantly time-dependent, particularly for orbits with periods that span a significant fraction of the age over which the parent galaxy formed. But even if the external potential is highly symmetric, a dwarf galaxy in a non-circular orbit will necessarily experience a time-variable potential as it traces its orbital path (Pinchardo et al. 2005). Clearly, many factors dictate the tidal history of any given galaxy, and, by implication, the degree to which a given system's kinematics are affected by tidal influences.

We know that in extreme cases these processes can have a transformative effect, converting dwarf galaxies into streams that encircle their parent galaxies (Ibata et al. 1994, 2001; Majewski et al. 2003). Whether less severe effects have been seen in other systems remains a matter of debate (e.g., Ursa Minor: Martínez-Delgado et al. 2001, Palma et al.

\footnotetext{
${ }^{1}$ We restrict ourselves here to cases in which Newtonian gravity applies. We will address the interpretation of dSph kinematics in cases assuming non-Newtonian gravity in a later paper.
} 
2003, Muñoz et al. 2005; Carina: Kuhn et al. 1996, Monelli et al. 2004; Majewski et al. 2005). As a population, local dwarfs exhibit trends, such as decreasing mass-to-light (M/L) ratio with increasing Galactocentric distance $\left(R_{G}\right)$ for Milky Way and M31 satellites (Mateo et al. 1998a; hereafter M98) and correlations of stellar ages in dwarf systems as a function of $R_{G}$ (van den Bergh 1994; Mayer et al. 2007), that provide circumstantial evidence tides do influence satellite properties to varying degrees.

Since our goal is to characterize the DM halos dwarf galaxies, it is clearly advantageous to try to identify galaxies that best exemplify systems in dynamical equilibrium. It is for this reason we (and others: M98; Sohn et al. 2007; Koch et al. 2007) have chosen to focus on the Leo I dSph galaxy. Located $255 \mathrm{kpc}$ from the Milky Way (Caputo et al. 1999; Held et al. 2001; Bellazzini et al. 2004), Leo I seems to be a good candidate as a truly isolated dwarf that is close enough for detailed kinematic study. This reasoning motivated M98 to obtain radial velocities for 33 red giants in Leo I from which they derived a central velocity dispersion. Under the assumptions of dynamical equilibrium and mass follows light, they argued that Leo I does contain a DM halo of comparable mass to those inferred in other, closer dSph galaxies. This result implied that tides are not the sole cause of the observed kinematic properties of dSph galaxies.

However, Leo I has two puzzling properties that suggest it may not be the ideal, dynamically-isolated system we would hope it to be. First, the heliocentric radial velocity, $v_{h}$ of Leo I is extreme $(282.9 \mathrm{~km} / \mathrm{s}$; see Section 4.3 .1$)$. Relative to the Galactic Center rest frame, it has a recessional velocity of $174.9 \mathrm{~km} / \mathrm{s}$ as observed from the Sun, very high for a remote satellite of the Milky Way. Indeed, Leo I can singlehandedly inflate the inferred mass of our Galaxy by nearly a factor of five compared to dynamical analyses of kinematic samples that exclude it (Zaritsky et al. 1989; Fich and Tremaine 1991; Kochanek 1996; Wilkinson and Evans 1999; Sakamoto et al. 2003). But the large velocity also has an important implication for Leo I itself. Standard cold DM (CDM) hierarchical formation models of the Milky Way tend to produce outward-streaming dwarfs at late times as the smaller galaxies begin to interact strongly with the dominant, massive central halo (Taylor et al. 2005) or other dark halos (Taylor and Babul 2004, 2005; Sales et al. 2007a,b). Is the high systemic velocity of Leo I direct evidence of a strong past interaction with the Milky Way or, possibly, some third body?

The second enigma is that Leo I has a complex star-formation (SF) history (Gallart et al. 1999b; Hernandez et al. 2000; Dolphin 2002), with the latest significant episode ending about 1 Gyr ago. Within the framework of the tidal-stirring models of Mayer et al. (2001a,b, 2005), periods of enhanced star formation represent times when the gas in a dwarf is compressed toward the center of its DM halo during strong interactions with the parent 
halo. Does the complex star formation history of Leo I reveal evidence of such an event presumably an interaction with the Milky Way - about 1 Gyr ago?

This paper presents new high-precision kinematic observations of over 300 individual member red giants in the Leo I dSph galaxy, significantly extending both the quantity, quality, and spatial coverage of earlier samples. With these new data it is possible to address whether Leo I represents an example of an isolated, equilibrium system, or if its dynamics are significantly influenced by external tidal effects.

\section{Observations and Data Reduction}

The principal data of this paper consist of new radial velocity measurements derived from spectra obtained using the multi-fiber Hectochelle spectrograph on the MMT telescope (Szentgyorgyi et al. 1998; Szentgyorgyi 2006). The observations were carried out during two nights dedicated to this project (March 31/April 1 and April 2/3, 2005), and during parts of six separate nights of two Hectochelle 'queue runs' in April, 2006 and March/April 2007 (see Table 1 for details). Seven different fiber configurations were used, denoted chronologically as ' $c 1$ ' through ' $c 7$ '. We purposely allowed for considerable target overlap in the various configurations to allow us to use repeat measurements to quantify the velocity uncertainties (Section 3.2). The spatial distribution of the targets is shown in Figure 1, along with all of the Leo I red giant $(\mathrm{RG})$ candidates.

Candidates stars to be observed with Hectochelle were selected from the $(I, V-I)$ colormagnitude diagram (CMD) of Leo I derived from CCD data obtained with the Hiltner $2.4 \mathrm{~m}$ Telescope at MDM Observatory in February, 2004, and for the outermost candidates, from data we obtained with the 90Prime camera on the Steward $2.3 \mathrm{~m}$ telescope at Kitt Peak in Feb 2006 (Williams et al. 2004). The locations of the all candidate stars in the CMD are shown in Figure 2. The photometric calibration was carried out using 22 stars in the field that have calibrated photometry from M98. The rms scatter of the adopted transformations is about $0.02 \mathrm{mag}$ in both $I$ and $(V-I)$. The astrometry for these stars was determined by transforming of the CCD coordinates to standard coordinates, $(\xi, \eta)$ using 200-400 USNOB/NOMAD (Monet et al. 2003) coordinates of stars in the field.

We chose regions devoid of detectable stars in our deep imaging to be used for spectroscopic 'sky' measurements. These sky fiber locations span the full radial extent of the target fibers relative to the center of Leo I. Additional 'sky' fibers were also randomly assigned by the Hectochelle fiber robot software at the time of observation to utilize otherwise idle fibers. Since these could, by chance, land on an astronomical source, no automatically-assigned sky 
fibers were used in our analysis. We also observed the radial velocity standard HD171232, as standards in the SA57 field (Stefanik et al. 2006). The Hectochelle fiber robot software generally assigned different fibers to these stars on separate visits. Further details of our Leo I and standard-star observations are provided in Table 1.

Hectochelle employs two $2048 \times 4096$ CCDs, illuminated by 244 fibers and read out through 4 amplifiers (2 on each CCD). Fibers are assigned to targets to avoid mechanical overlaps and collisions, while maximizing user-specified priorities, and can be positioned anywhere within a 1-degree diameter field. The fibers terminate at the focal plane of the collimator of an echelle spectrograph located on an optical bench near the telescope. To increase the packing factor on the CCD, the fibers are mounted in a zigzag pattern at the slit plane. Thus, consecutive spectra are offset in both the spatial and dispersion directions from one another. The entire pattern also exhibits slight curvature along the 'spatial' axis due to anamorphic effects in the spectrograph optics. To avoid order overlap, an interference filter was used to isolate the wavelength region of interest. We observed our targets through the 'RV52' filter which covers the region of the Mg I/Mgb features around $5200 \AA$. Further technical information on Hectochelle is available at (Szentgyorgyi 2006) and from the user's manual2.

IRAF 3 routines were used to process the raw images, extract and wavelength-calibrate the individual spectra, and to measure the final heliocentric velocities. The procedures are broadly similar to ones we have employed in the past for single-object slit spectroscopy (e.g. Vogt et al. 1998) and for multi-fiber spectroscopy of Southern dwarf galaxies (Walker et al. 2007a). We briefly summarize them here.

All data were processed by first subtracting the overscan, trimming the images, then combining the data from the two amplifiers for both CCDs. Individual exposures were combined to form single deep images and to remove the brightest point-like cosmic rays in the raw data using a conservative sigma-clipping algorithm. Hectochelle had a source of significant scattered light within the spectrograph chamber during the 2005 run (configurations c1 and c2 in Table 1). This problem made it impossible to flatfield these data or to perform background subtraction. We estimate the scattered light cost us about one magnitude in depth compared to the 2006/2007 observations, all of which were unaffected by this problem.

For the 2006 and 2007 observations (configurations c3 through c7 in Table 1), we deter-

\footnotetext{
${ }^{2}$ http://cfa-www.harvard.edu/mmti/hectospec/Hectochelle_Observers_Manual.pdf

${ }^{3}$ IRAF was distributed by the National Optical Astronomy Observatories, which are operated by the Association of Universities for Research in Astronomy, Inc., under cooperative agreement with the National Science Foundation.
} 
mined relative fiber throughputs from observations of the twilight sky. Quartz lamp spectra were inadequate for this as the light sources on the telescope do not illuminate the fibers uniformly. After extraction, wavelength calibration (see below) and dividing by the relative throughputs, we then combined all the sky fiber spectra to make a master sky for each configuration. This combined sky spectrum was subtracted this from the spectrum of each target and each individual sky spectrum. The master sky spectrum was typically produced by averaging 40-60 individual spectra, so it adds little variance to the final sky-subtracted spectra. Overall, this procedure appears to have worked well: Individual sky fibers had little mean residual flux after subtraction (typically $\leq 5 \%$ of the original sky flux). In contrast to the 2005 data, cross-correlations of both target and sky fibers rarely produced a signal at the expected velocity of scattered sunlight at the time of observations (see Figure 3).

We established the locations of the individual spectra on the detectors by tracing and extracting spectra of quartz lamps obtained just before or after each set of observations for a given configuration. The spectral traces from the quartz images were then shifted spatially en masse to best match the fainter spectra of the corresponding target exposures. The shifts of the quartz spectra to the target spectra were stable to about 0.01 pixel, as determined by comparing results for individual target exposures of a given configuration. ThAr calibration emission spectra were extracted using the same traces used for the corresponding target spectra. A dispersion solution for each arc spectrum was determined by fitting the centroids of 35-40 lines with known wavelengths to a fifth-order polynomial. The fits had a typical RMS scatter of about $0.3-0.5 \mathrm{~km} / \mathrm{s}$. The resulting dispersion solutions were then applied to all spectra, producing wavelength-calibrated, one-dimensional spectra for every fiber. The spectra are defined from $5150 \AA$ to $5300 \AA$, with an effective dispersion of $0.01 \AA /$ pix $(R \sim 25,000)$. Spectra of the standard stars were processed in essentially the same way except that these were sufficiently bright to be traced without need of a quartz exposure.

\section{Velocity Measurements}

\subsection{Velocity Standards}

We constructed a high signal-to-noise master template spectrum from individual spectra of radial velocity standard stars observed during the 2005 MMT run (see Table 1). We chose HD171232 to act as a 'master' template, then used the Fourier cross-correlation routine fxcor in the $r v$ package of IRAF to measure relative shifts of this template and all the standardstar spectra. Our results for each standard-star observation spectrum are listed in Table 2, based on an assumed heliocentric velocity of HD171232 of $V_{h, H D 171232}=-37.3 \pm 0.8 \mathrm{~km} / \mathrm{s}$ (Udry et al. 1999). We also list the heliocentric velocities for the standard stars from SA57 
(Stefanik et al. 2006). The individual standard-star spectra were Doppler-shifted by their observed velocity shift relative to HD171232, then summed to make a master template with a mean signal-to-noise ratio of about 400:1 per resolution element. At this stage, the final template was tied to the velocity scale defined by HD171232 given the Udry et al. (1999) velocity.

A problem became evident when we found that the SA57 standards (Stefanik et al. 2006) from all runs exhibit an offset of $\Delta_{S A 57}=-3.4 \pm 1.3 \mathrm{~km} / \mathrm{s}$ when tied to HD171232 (see Table 2). We suspect this offset is not associated with the SA57 standards for the following reasons. First, the mean heliocentric velocity of sky fiber spectra with a Tonry-Davis (1979) index, $R_{T D}$, greater than 2.8 (see section 3.2 ) was $-4.1 \pm 0.8 \mathrm{~km} / \mathrm{s}$, consistent with $\Delta_{S A 57}$, though not with zero. Second, during the 2006 run, three sets of twilight exposures were obtained. These data gave a mean heliocentric velocity of $-3.4 \pm 0.2 \mathrm{~km} / \mathrm{s}$ for the twilight spectra using the HD171232 velocity zeropoint; again consistent with $\Delta_{S A 57}$ but not with zero. Third, velocity measurements from other configurations where the spectra of faint stars are overwhelmed by moonlight were found to be offset by $-3.7 \mathrm{~km} / \mathrm{s}$ using the HD171232 zeropoint. Finally, Udry et al. (1999) remark that the velocity of HD171232 appears to 'drift', a result we appear to confirm.

We can reconcile HD171232, the SA57 standards and the night-sky and twilight spectra by simply shifting our adopted velocity scale by $-\Delta_{S A 57}=+3.4 \mathrm{~km} / \mathrm{s}$. We did this by amending the heliocentric velocity in the master template spectrum by $+3.4 \mathrm{~km} / \mathrm{s}$, effectively adopting $v_{\text {helio }}=-33.9 \mathrm{~km} / \mathrm{s}$ for HD171232. With this change, the heliocentric twilight and night-sky velocities all averaged to within $\pm 1 \mathrm{~km} / \mathrm{s}$ of zero, and the velocities of the SA57 standards came into excellent agreement to their published values (Stefanik et al. 2006; Table 2). We believe that our final heliocentric velocity zeropoint is systematically accurate to $\leq 1 \mathrm{~km} / \mathrm{s}$.

\subsection{Velocities of Leo I Candidates}

We used fxcor in IRAF to measure the velocities of Leo I candidates relative to the template. Typical cross-correlation functions are shown in Figure 3. Tonry and Davis (1979) defined a parameter, $R_{T D}$, which measures the height of the cross-correlation peak relative to the amplitude of the noise in the cross correlation function near the peak. The fxcor task reports $R_{T D}$ for all spectra for which it estimates a velocity. All of the spectra used here have $R_{T D} \geq 2.8$. This cutoff represents the value of $R_{T D}$ where it became difficult to identify consistently a correlation peak, and where quantitative comparisons of independent measurements of individual stars revealed that our velocity measurements were becoming 
unreliable (see below).

Some statistics for our MMT/Hectochelle fiber observations are provided in Table 3. Of the 749 fibers assigned to astronomical (i.e., non-sky) targets in the five Leo I configurations, we obtained 543 spectra (72\%) of 371 different targets that produced cross-correlations with $R_{T D} \geq 2.8$. About $59 \%$ of our assigned fibers (440 spectra) produced good velocities for the final sample of 312 likely Leo I members observed with Hectochelle (membership is quite clear-cut in the case of Leo I as described below). If we add the 33 targets from M98 (we justify this below), our final sample consists of 387 stars, 328 of which are likely Leo I members (17 of the M98 stars were reobserved with Hectochelle). A total of 108 stars (297 spectra) were observed multiply, with 51 stars twice, 38 three times, 15 four times, and 3 five times. One star (number 359 in Table 5) was observed on six separate occasions. Table 4 gives a complete listing of all the repeat measurements within the combined MMT/Keck dataset.

We have used the repeat observations to assess the quality of our velocity measurements. Figure 5 plots the histogram of the velocity differences in our dataset, along with plots of the velocity differences as a function of position, velocity, and brightness. There are no significant correlations apparent, nor do we see evidence that different configurations are offset relative to one another.

Repeat measurements can also be used to estimate the individual velocity errors as described by Walker et al. (2006a). We assume a relation between the velocity error, $\sigma_{i}$, and $R_{T D, i}$ for star $i$, of the form

$$
\sigma_{i}^{2}\left(R_{T D, i}\right)=\left(\frac{\alpha}{\left(1+R_{T D, i}\right)^{x}}\right)^{2}+\sigma_{0}^{2}
$$

The best-fit parameters $\alpha, x$ and $\sigma_{0}$ are determined via a least-squares minimization process (Walker et al. 2006a; 2007) from which we obtained $\alpha=2.60 \mathrm{~km} / \mathrm{s}, x=0.16$, and $\sigma_{0}=0.14$ $\mathrm{km} / \mathrm{s}$. In calculating these parameters, we did not vet the sample in any way. Astrophysical sources of velocity variations (e.g. atmospheric or binary motions) will contribute to our estimates of the individual velocity errors. Our data do indeed reveal evidence of possible binaries (Section 3.4), but neither the magnitude of the velocity variations nor the frequency of detectable binaries significantly alter our results (Section 4.1). The distribution of $\sigma\left(R_{T D}\right)$ from repeat measurements, is quite flat, probably owing to systematic run-to-run velocity errors at the $1-2 \mathrm{~km} / \mathrm{s}$ level. These represent a negligible contribution to our final error budget since, even with a catalog of $300+$ Leo I members, our kinematic results are still dominated by sampling uncertainties (Sections 3.3, 4.2).

For the stars with multiple observations, we used these error estimates to determine the 
mean velocity for each star. We assumed that the $n$ multiple, independent velocity measurements $\left\{v_{1}, \ldots, v_{n}\right\}$ of a star with true velocity $u$ follow a Gaussian distribution centered on $u$. From maximum likelihood statistics (Rice 1995), the estimate of $u$ is given by the weighted mean: $\hat{u}=\sum_{i=1}^{n}\left(v_{i} \sigma_{i}^{-2}\right) / \sum_{i=1}^{n}\left(\sigma_{i}^{-2}\right)$. The velocity range that includes $u$ with probability $1-\gamma$ is then given by $\hat{u} \pm n^{1 / 2} t_{n-1}(\gamma / 2) S$, where $t_{n-1}$ is the $t$ distribution with $n-1$ degrees of freedom and $S^{2} \equiv(n-1)^{-1} \sum_{i=1}^{n}\left(v_{i}-\hat{u}\right)^{2}$. For each star with multiple measurements, we calculate $\hat{u}$ and its $1 \sigma(\gamma=0.32)$ confidence interval. Figure 6 is a histogram of the the velocity differences of repeat measurements $\left(\Delta_{2} \equiv v_{i}-\langle v\rangle\right)$ plotted in bins of $\mathrm{km} / \mathrm{s}$ and in units of the velocity error, $\sigma\left(\Delta_{3} \equiv \Delta_{2} /(\sqrt{2}\langle\sigma\rangle)\right.$. The Gaussian profile in the lower histogram is the expected distribution for $n=2$ and $\sigma_{i}=\sigma_{j}$, which is reasonably valid for about $70 \%$ of the cases plotted in the histogram.

The final adopted velocities for 387 stars in our Leo I dataset are listed in Table 5, excluding velocities from sky fibers and for any spectra with $R_{T D}<2$. . This table includes results for stars from M98, adopting the velocity uncertainties tabulated in that paper. Table 5 also lists positions and photometric data for each star and the configurations used to determine their velocities. For stars with a single observations (and a known value of $\left.R_{T D, i}\right)$, velocity uncertainties are $\sigma_{T D, i}$ from the equation above. For stars with multiple observations, the velocity uncertainties are obtained from the $68 \%(1 \sigma)$ confidence limits as described in the previous paragraph.

Sohn et al. (2007; hereafter S07) carried out an independent kinematic study of Leo I based on near-IR spectra of resolution $R \sim 3000$ obtained with DEIMOS on the Keck telescope. Using a 0.5 arcsec matching radius, we find 26 stars from S07 in common to our sample. Figure 7 summarizes the velocity differences, $\Delta$ in the same format as Figure 5 . The lower-left panel of Figure 7 reveals a strong systematic trend of $\Delta$ with velocity. The coefficients of the fitted line are given in the figure caption. The correlation coefficient indicates that a better linear fit could occur by chance less than $0.02 \%$ of the time for a sample this size. The slope of this trend $(-0.31 \mathrm{~km} / \mathrm{s}$ per $\mathrm{km} / \mathrm{s})$ is a significant contributor to the width of the histogram of $\Delta$ in the upper-left panel of Figure 7 . The standard deviation of $\Delta$ about the fitted line is $3.5 \mathrm{~km} / \mathrm{s}$ while the standard deviation of the data in the histogram is $4.8 \mathrm{~km} / \mathrm{s}$. We find no significant correlation of velocity difference between our data and that of S07 as a function of position or I-band brightness.

Since the comparisons of our MMT and Keck datasets reveal no systematic trends, we tentatively conclude that the source of the systematic trend lies in S07 measurements. Many authors have noted special complications with velocity measurements using near-IR spectra due to telluric contamination (a summary of this is given in Walker et al. 2007). If the nearIR spectra are indeed the principal source of the trend in Figure 7 , the S07 velocities are 
offset by about $+10 \mathrm{~km} / \mathrm{s}$ at the low end of the Leo I velocity range (approximately 250-310 $\mathrm{km} / \mathrm{s}$ on the MMT velocity scale) and by about $-2 \mathrm{~km} / \mathrm{s}$ at the upper end of the range. Given the complications and uncertainties involved with dealing with this trend, the unusual spatial coverage of targets observed by S07 (due to constraints associated with DEIMOS slit masks), and the modest number of new Leo I members we would gain from the S07 sample (an additional 15\% at most to our sample), we have chosen not to include the S07 results in our analysis.

Koch et al. (2007; hereafter K07) have also recently published a kinematic study of Leo I based also on moderate-dispersion, near-IR spectroscopy. Using a 5 arcsec matching radius (K07 report their coordinates to a precision of only 1 arcsec) and requiring $10 \%$ photometric matching, we find 17 stars in common to the two samples. Figure 8 summarizes the velocity differences, $\Delta$, plotted in the same format as Figure 5. Apart from a systemic offset (see upper left panel), we find no significant trend in the comparison of these velocities, nor as a function of stellar brightness. In part because of this systematic offset, but mostly since the typical velocity uncertainties of the K07 sample are about twice those of our new measurements, we have chosen not to merge the datasets here.

Figure 9 is a plot of all velocities measured from our sample for spectra with $R_{T D} \geq 2.8$, as a function of radial distance from the adopted center of Leo I (Mateo 1998; see Figure 1). The results in Table 5 are plotted as filled points, while velocities measured from sky fibers (but still with $R_{T D} \geq 2.8$ ) are shown as open symbols. There is a clear concentration of stars around the mean systemic heliocentric radial velocity of Leo I $(282.9 \mathrm{~km} / \mathrm{s}$, Section 4.3 .1 ; Zaritsky et al. 1989; M98). The stars in this velocity range also exhibit a clear concentration toward the center of Leo I. When we also consider the expected velocity distribution of field stars in this direction (Robin et al. 2003), it is clear that all stars with $V_{\text {helio }}$ in the range 250$320 \mathrm{~km} / \mathrm{s}$ are highly probable Leo I members (the range $200-400 \mathrm{~km} / \mathrm{s}$ would have identified precisely the same sample of likely members). Stars with velocities outside this range are uniformly distributed spatially in the field, consistent with non-membership (see Figure 1).

Sky fibers with $R_{T D} \geq 2.8$ tend to exhibit a mean velocity of about $-19.7 \pm 0.8 \mathrm{~km} / \mathrm{s}$ when plotted in Figure 9 because fxcor assumes the target is an astronomical object and applies a heliocentric correction. For our Leo I observations, the heliocentric correction for a Leo I field was always about $-19 \pm 2 \mathrm{~km} / \mathrm{s}$. Although only non-sky fiber results are plotted in Figure 9, many velocities cluster around $-19 \mathrm{~km} / \mathrm{s}$. These are likely 'false positives' where the target stars were too faint to produce usable spectra, but for which the sky velocity could be measured. We somewhat arbitrarily define false positives as cases where we observe a heliocentric velocity in the range -10 to $-28 \mathrm{~km} / \mathrm{s}$ and $R_{T D} \leq 4.2$. There are 14 such cases in Figure 9, all of which are noted in Table 5. Each has data from only the 2005 dataset 
(which we could not sky-subtract). Moreover, many false positives based on 2005 spectra alone turned out to have well-determined, non-sky velocities obtained from spectra from the 2006 or 2007 runs, or from the M98 sample. The converse of this effect - sky spectra that scatter into the acceptance range for Leo I members $(250-320 \mathrm{~km} / \mathrm{s})$ - never occurred in multiply-observed data with 2005 observations. Given the narrow distribution of velocities we observe in the sky fibers, such scatter is statistically extremely unlikely.

\subsection{Velocity Dispersion Profiles}

Merritt and Saha (1993) and Wang et al. (2005) have described non-parametric approaches that produce dispersion profiles without binning. These methods are marginally appropriate for the present Leo I data set because the sample size ( $N=328$ members) remains rather small. We will describe a non-parametric analysis for Leo I and other dSph galaxies in a separate paper (Walker et al. 2007, in preparation).

For now, we adopt here the more standard approach of using binned profiles in our analysis. The bins in our profiles contain (nearly) equal numbers of stars as dictated by the sample size and number of bins, $N_{b i n}$. Profiles for $N_{b i n}=15,20$ and 25 are shown in Figure 10, The horizontal 'error bars' in the profiles show the standard deviation in $R$ for the stars in each bin. A common problem with binning is the possibility that false structures may be produced in the profiles. Walker et al. (2006b) have investigated this problem and find that bins contain 10 or more stars seem to be systematically stable to within the calculated Poisson uncertainties. From top to bottom in Figure10, the three binning options correspond to $21 / 22\left(N_{\text {bin }}=15\right), 19 / 20\left(N_{b i n}=20\right)$ and $13 / 14\left(N_{b i n}=25\right)$ stars per bin. There are no significant features in any of the profiles that are not visible in the other (this remains true if we offset the bins), so these binning options appear to be fairly robust. Since our aim is to model the Leo I dispersion profile with simple dynamical models, we will take advantage of the higher $\mathrm{S} / \mathrm{N}$ per bin of the $N_{\text {bin }}=15$ profile and use this one exclusively in our subsequent analysis. The smaller, downward error bars on the dispersions in Figure 10 are based on the method described by Kleyna et al. (2004), while the larger, symmetric error bars are calculated using the method described by Walker et al. (2006a, 2007a).

\subsection{Temporal Stability}

With the inclusion of the M98 results, the subset of stars in Table 5 with multiple observations spans slightly over 11 years of temporal coverage. The possibility of kinematic 
variability in our sample is suggested by the outliers apparent in the lower panel of Figure 6 , To explore this further, we produced Figure [11, a plot of the reduced chi-squared, $\chi_{\nu}^{2}$ for all stars with multiple measurements for $\nu$ degrees of freedom. The lines show the values of $\chi_{\nu}^{2}$ corresponding to a $0.5 \%$ probability of exceeding the value of $\chi_{\nu}^{2}$ by chance for $\nu=1$ (2 observations) to 4 . Seven stars, including five Leo I members, exhibit values of $\chi_{\nu}^{2}$ that suggest they may be binaries. In every case, the stars have only two observations and exhibit $\Delta V \leq 13.3 \mathrm{~km} / \mathrm{s}$. These stars are noted in Tables 4 and 5 .

The two stars with the smallest heliocentric velocities have $\chi_{\nu}^{2} \sim 9$, and, in both cases, the observations were obtained only a few days apart. As neither are members of Leo I, they can plausibly have short orbital periods. In the other cases (all Leo I members; see Tables 4 and 5), the time intervals between observations range from as little as 1 to at most 10 years. For a sample of 102 stars with repeat measurements, we might reasonably expect 1-2 stars to exceed the $0.5 \%$ line in Figure 11 by chance. The presence of five outliers suggests we have detected at least a few physical binaries in Leo I.

We can look for more subtle evidence of binarity by splitting the sample of multiplyobserved stars into subsamples with different time intervals between the individual observations. One subsample consists of stars observed multiply in a single run. We take these to have a time interval between observations, $\Delta t$, of zero since plausible red giant binaries in Leo I must have orbital periods long compared to the length of a single run. The second subgroup contains stars observed either one or two years apart (that is, during different MMT/Hectochelle runs; see Table 1) $\Delta t \sim 1-2$ yr. Finally, stars observed with the MMT in 2005-07 and during the 1996 Keck observations published by M98 have $\Delta t$ of 9 or 10 years and comprise a final subgroup (there are no Keck repeats from the $2007 \mathrm{MMT}$ run). For the $\Delta t=0$ subgroup we find a mean velocity difference of $\left.\left\langle\Delta v_{(0} \mathrm{yr}\right)\right\rangle=0.58 \pm 0.37 \mathrm{~km} / \mathrm{s}$, with $\mathrm{rms}$ of $\sigma_{(0 \mathrm{yr})}=3.4 \mathrm{~km} / \mathrm{s}$, and $N=87$. For the $\Delta t=1 \mathrm{yr}$ subgroup the values are $\left\langle\Delta_{(1 \mathrm{yr})}\right\rangle=0.10 \pm 0.37, \sigma_{(1 \mathrm{yr})}=4.2 \mathrm{~km} / \mathrm{s}$, and $N=138$. Finally, for the long-interval subgroup we find $\left.\left\langle\Delta_{(10 \mathrm{yr})}\right\rangle=-0.29 \pm 0.66, \sigma_{(10} \mathrm{yr}\right)=3.8 \mathrm{~km} / \mathrm{s}$, and $N=33$. These values are consistent with no discernable change in $\sigma_{(\Delta t)}$ as time interval increases.

\section{The Dynamics of Leo I}

In this section, we explore two cases that serve as frameworks to interpret our new kinematic data for Leo I. The first corresponds to the case where the stellar and dark components of Leo I are in dynamical equilibrium, while the second explores the possibility that some or none of the dynamical components that comprise Leo I are in dynamical equilibrium. For convenience, various parameters for Leo I that we use or derive in this section are 
summarized in Table 6.

\subsection{Binary Stars}

Before we can discuss dynamical models for Leo I, we must address the potential contamination by spectroscopic binaries. In general, orbital motions in binaries will enhance the velocity dispersion of a kinematic sample. We found above that our dataset reveals at most seven possible binaries (5 Leo I members) among the 108 stars (84 Leo I members) with multiple observations. Are these binaries present in sufficiently large numbers and with sufficiently large velocity excursions to significantly affect our interpretation of the galaxy's kinematics?

Red giants in Leo I that reside in binaries with periods up to a few hundred years will exhibit maximum velocity amplitudes comparable to the internal dispersion we measure for the galaxy $(\sim 9 \mathrm{~km} / \mathrm{s})$. Projection (inclination) effects, a spread in binary mass ratios, and a range of orbital separations all tend to reduce the velocity amplitudes one actually observes in a realistic sample. A hint that suggests binaries are unimportant in our Leo I data is our evidence (Section 3.4 above) that the sample dispersion does not change with increasing time baseline interval. Our observations are consistent with a population of binaries that contributes a 'dispersion' comparable to or smaller than the mean measurement errors, about $2-3 \mathrm{~km} / \mathrm{s}$.

Previous studies have addressed the issue of binary contamination in kinematic samples of dSph galaxies (Hargreaves et al 1996; Olszewski et al. 1996), taking into account plausible period, inclination and mass distributions for binary populations that amount to some fraction, $f_{b}$, of the total sample. Here, $f_{b}$ is defined here as the total number of apparently single stars that are actually unresolved binaries, divided by the total number of apparently single stars. The simulations of Olszewski et al. (1996) for the case of $N=17$ and a population with an intrinsic dispersion of about $7 \mathrm{~km} / \mathrm{s}$ are, remarkably, appropriate for single bins in our Leo I dispersion profile (Figure 10). From their results (Table 9 of Olszewski et al. 1996) we find that even for an extreme case with $f_{b}=0.7$ and all binaries distributed within the shortest period (highest velocity amplitude) range of 0.5-100 yrs, a single-epoch measurement will typically overestimate the true dispersion by only about $10 \%$, with the $95 \%$ confidence interval ranging between about $4.5-10 \mathrm{~km} / \mathrm{s}$ for an assumed sample dispersion of $7 \mathrm{~km} / \mathrm{s}$.

In Leo I, we observe a lower limit to the binary frequency of $f_{b} \geq 5 / 84 \sim 0.06$ for a set of observations that was sensitive to binaries a period range of a few days to few decades. The 
lack of an increase in dispersion with time baseline suggests there is no significant population of longer-period binaries lurking under the radar. Thus, for Leo I the likely binary frequency appears to be much lower than the $70 \%$ frequency assumed in the simulation from Olszewski et al. (1996) we cited above. We conclude that binaries have inflated the dispersions of single bins in Figure 10 by at most $\sim 10 \%$. Given that the typical error bars on these individual dispersions are around 15-20\%, the effects of binaries are negligible for any given bin. To be safe, we use the larger symmetric error bars from Walker et al. (2006a, 2007a; see Figure 10) to calculate goodness-of-fit in all subsequent analyses.

\subsection{Equilibrium Models}

Figure 12 shows a comparison of the velocity dispersion profile of Leo I (for the $N_{b i n}=15$ profile) with an isothermal model $(\sigma=9.2 \pm 0.4 \mathrm{~km} / \mathrm{s}$; see Section. 4.3.1 and Table 6), a single-component King (1966) dynamical model, and a two-component Sersic+NFW (Sersic 1968; Navarro et al. 1997; Łokas 2002) model. The core radii of the isothermal and King models is taken to be that of the visible stellar distribution $\left(R_{\text {core }}=245 \mathrm{pc}\right.$ for an assumed distance of $255 \mathrm{kpc}$ ) and a concentration parameter of $c \equiv \log \left(R_{\text {tidal }} / R_{\text {core }}\right)=0.6$ (Irwin and Hatzidimitriou 1995; hereafter IH95). The Sersic profile used with the NFW model is assumed to be concentric with the DM halo. We followed the recipe of Łokas (2002), adopting a Sersic profile index of $m_{S}=0.6$ and a Sersic radius of $r_{S e r s i c}=370 \pm 30$ pc (see Figure 17). We assumed $M / L=1.0$ for the visible matter, implying $\left.M_{\text {visible }}=5.6 \pm 1.8 \times 10^{6} M_{\odot}\right)$. Models with anisotropy parameter, $\beta$ (see Binney and Tremaine 1987), ranging from 0.0 (isotropic) to -3.0 (moderately tangentially aniostropic) are compared to the observations in Figure 12, For a given model, $\beta$ is constant.

Because the observed dispersion profile of Leo I is so flat, the isothermal sphere (top panel of Figure 12) provides a good fit to the kinematic data. For our assumption that the mass distribution has the same core radius as the visible matter, the central density for the isothermal case is $0.23 \pm 0.04 M_{\odot} \mathrm{pc}^{-3}$ (Richstone and Tremaine 1986; M98). The baryonic

central density is considerably lower, $\rho_{b} \sim 0.02-0.05 M_{\odot} \mathrm{pc}^{-3}$ for $(M / L)_{V, \text { baryons }}=0.3-0.7$ (M98; below), where we have converted the projected surface density assuming a King profile with parameters from IH95 corrected to an adopted distance of $255 \mathrm{kpc}$. The mass of the best-fitting spherical, isothermal sphere out to $1040 \mathrm{pc}(\sim 840 \mathrm{arcsec}$, the location of the outermost kinematic member of Leo $\mathrm{I}$ in our sample) is $5.2 \pm 1.2 \times 10^{7} M_{\odot}$, implying $M / L=9.3 \pm 4.0$ (in Solar units) interior to this radius. The projected mass density of the isotropic model does not resemble the visible mass density (see Figure 17), but this just means that mass does not follow light. In particular, $\rho_{D M}>>\rho_{v i s}$ everywhere in Leo I, with 
the DM distribution considerably more extended than the visible matter.

It has long been known that King (1966) models provide a good fit to the visible matter distribution in Leo I and other dSph galaxies(IH95). However, a single-component King model in which mass follows light (middle panel of Figure 12) fails spectacularly to fit the observed dispersion profile. For any King model that fits the light distribution, the predicted dispersion begins to decrease steadily outside the core radius, falling well below the observed profile, reaching zero at the tidal radius (by design, of course). Through its failure to fit the kinematics of Leo I, the King model also implies that, in equilibrium, the mass distribution of the galaxy must be considerably more extended than that of the visible matter.

A two-component Sersic+NFW model does considerably better at accounting for the dispersion profile and the visible matter distribution, particularly if we allow for some (radially constant) kinematic anisotropy (lower panel, Figure 12). The isotropic case, $\beta=0$, cannot simultaneously fit the inner and outer parts of the dispersion profile for any assumed Leo I mass. The best fit is for $\beta=-1.5$ and $M_{v i r}=7 \pm 1 \times 10^{8} M_{\odot}$, where $M_{v i r}$ is the mass interior to the virial radius (defined here as the radius where $\rho\left(R_{\text {vir }}\right)=200 \rho_{\text {crit }}$ ). The $95 \%$ confidence interval on $\beta$ ranges from -0.4 to -3.2 . The best-fit Sersic + NFW model implies a mass of $8.1 \pm 2.0 \times 10^{7} M_{\odot}$ interior to $1040 \mathrm{pc}$, the radius of the outermost Leo I member in our kinematic sample. This mass is about $60 \%$ higher than the mass obtained from the isothermal model (above; see Table 6). At this radius, $M / L=14.4 \pm 5.8$ (in Solar units) for the Sersic+NFW case.

\subsection{Are Equilibrium Models Valid for Leo I?}

The large heliocentric velocity of Leo I and its unusual star formation history suggest that the galaxy may have experienced a strong encounter with the Milky Way in the not-toodistant past. Here we explore the interpretation of our new kinematic results in the context of such an interaction.

\subsubsection{The Radial Velocity of Leo I}

From the entire sample of 328 Leo I members, we find a weighted $\left(w_{i}=1 / \sigma_{i}^{2}\right)$ mean heliocentric velocity of $282.9 \pm 0.5 \mathrm{~km} / \mathrm{s}$ and a sample dispersion of $\sigma=9.2 \pm 0.4 \mathrm{~km} / \mathrm{s}$. For an assumed motion of the Local Standard of Rest (LSR) of $220 \mathrm{~km} / \mathrm{s}$ toward an apex at $(l, b)=(90,0)$ and a peculiar solar motion relative to the LSR of $16.6 \mathrm{~km} / \mathrm{s}$ toward $(l, b)=(53,25)$, Leo I has a velocity of $174.9 \pm 0.5 \mathrm{~km} / \mathrm{s}$ for an observer located at the Sun 
but stationary with respect to the Galactic Center (we refer to this as the 'Galactostationary', or 'GS', reference frame). The angular separation of the Sun and Galactic Center as seen from Leo I is only $1.7 \mathrm{deg}$, so the GS radial velocity of Leo I must be very close to the Galactocentric radial velocity component of Leo I for any tangential velocity that keeps Leo I bound to the Milky Way. Leo I is fairly compact on the sky, so the sample dispersion is unaffected by this change of reference frame. Hence, $\sigma_{G S}=\sigma_{\text {helio }}=9.2 \pm 0.4 \mathrm{~km} / \mathrm{s}$.

The large outward velocity of Leo I has long been problematic and puzzling. Analyses of the total mass of the Milky Way using halo tracers (e.g. Zaritsky et al. 1989; Kochanek 1996; Wilkinson and Evans 1999; Sakamoto et al. 2003) are often strongly affected by the inclusion of Leo I. Taylor et al. (2005) pointed out that a system with kinematics similar to Leo I is rarely seen in CDM simulations of the formation and late-time evolution of the Local Group. Byrd et al. (1994) suggested that Leo I is unbound to the Milky Way (but bound to the Local Group), postulating that the dwarf originated closer to the Andromeda galaxy than the Milky Way. In this scenario, Leo I follows a hyperbolic trajectory relative to our Galaxy, exceeding the local escape velocity of the Milky Way at its current position.

Whether bound or unbound, the sign of Leo I's radial velocity means the galaxy was much closer to the Galactic Center in the past. In the preferred model of Byrd et al. (1994), $R_{\text {peri,LeoI }}=70 \mathrm{kpc}$, similar to the distances of the closest present-day dSph galaxies from the Galactic Center. Orbits for which Leo I is bound to the Milky Way imply even smaller perigalactica. This result is remarkable in that it implies Leo I entered the 'no-fly zone' ( $R \leq 60-70 \mathrm{kpc}$; Mayer et al. 2001a,b) of the Milky Way, a volume in which we find no internally bound dSph systems (Mateo 1998; Grillmair 2006; Belokurov et al. 2006). If Leo I passed through this no-fly zone, it would have experienced tidal forces of sufficient strength that, given enough time, are able to destroy dwarf systems of comparable luminosity.

To explore this further, Dr. C. Pryor kindly calculated a series of simple bound orbits for a point-like Leo I model in a logarithmic Galactic potential. These models suggest that Leo I passed closest to the Galactic Center 0.5-2 Gyr ago for $v_{t a n} \leq 100 \mathrm{~km} / \mathrm{s}$ in a Galactocentric rest frame. One such model, based on the orbital pole suggested by S07, is shown in Cartesian projection in Figure 13, while $R(t)$ is plotted in Figure 14. Within the framework of tidal stirring models (Mayer et al. 2001a,b; 2005), this most recent close passage to the Galactic Center would have been responsible for Leo I's latest significant episode of star formation (which ended about 1 Gyr ago; Gallart et al. 1999b; Hernandez et al. 2000; Dolphin 2002), and possibly may have stripped the galaxy of gas, completing its transformation into a spheroidal system. The important issue this raises is whether tidal interactions have left an imprint on Leo I. If so, what is the nature of the imprint and how does it affect our dynamical analysis? 


\subsubsection{Tidal Imprints in Leo I: Structural Properties}

It is well established that encounters between dwarf spheroidal galaxies and the Milky Way can raise strong tides in the smaller systems (Oh et al. 1995; Piatek and Pryor 1995; Read et al. 2006b; Klimentowski et al. 2006). One result is that debris from the dwarf is spread along the galaxy's past and future orbit. In the case of Leo I, its large distance and likely highly eccentric orbit (see below) would cause tidally-stripped matter - which we refer to loosely as 'tidal arms' - to project closely onto the main body of the dwarf.

The extent and structure of tidal arms depends sensitively on the dwarf mass. At one extreme, Kuhn and Miller (1989), Kuhn et al. (1996), Klessen and Kroupa (1998) and Fleck and Kuhn (2003), among others, have explored the effects of tides on interacting dwarfs that contain no dark matter at all. In such cases, prominent tidal arms are produced that create clear structural and line-of-sight kinematic signatures (e.g. Klessen and Kroupa 1998; Klessen et al. 2003; Read et al. 2006b). For example, systems with significant tangential velocities may produce tidal extensions visible along the projected orbital path on the sky. These may be seen as S-distortions in the projected stellar distribution (Odenkirchen et al. 2002; Grillmair and Dionatos 2006), or as 'breaks' in the light profiles (Read et al. 2006b). If the tangential velocity is small compared to the radial velocity component (as is likely the case for Leo I), the geometry of the orbit may cause arms to project mostly onto the main body of the disrupting dwarf. For this case, an observer may fail to notice striking structural anomalies (Read et al 2006b), even though significant line-of-sight extension is present. For Leo I, observations of the red giant branch and red clump (Gallart et al. 1999a; Bellazzini et al. 2004) and of RR Lyr stars (Held et al. 2000, 2001) reveal no compelling evidence that a significant fraction of the galaxy extends more than $\pm 15 \%$ (or about $\pm 40 \mathrm{kpc}$ ) from its main body. Similar observations have ruled out significant line-of-sight extensions in other local dwarfs (Klessen et al. 2003).

We conclude that if Leo I had a close encounter with the MW (say, as implied by the

orbit shown in Figures 13 and 14), then the lack of observable depth in Leo I and the absence of prominent structural anomalies require the presence of dark matter in Leo I. Otherwise, the galaxy would have been disrupted or greatly distorted in the encounter given its deep incursion into the Milky Way's 'no-fly zone' (Mayer et al. 2001b). But the presence of DM does not mean Leo I would have been unaffected by the encounter, just that tidal effects may be considerably subdued compared to the no-DM cases considered above.

To search for subtle structural tidal features, we have used our photometric observations to try to identify distortions in the projected geometry of Leo I. Figure 15 shows contours fit to a greyscale representation of the star counts of 12630 stars chosen within the boundary shown in the Leo I CMD (Figure2), and smoothed with a Gaussian kernel with $\sigma=2$ arcmin. 
By selecting stars from the CMD, we reduce field star contamination to about 1-2\% of the sample. The contours are independent fits to different isopleths of the stellar distribution for ellipses with semi-major axes ranging from 2-12 arcmin, in 1 arcmin intervals. These contours reveal no striking distortions or asymmetries, though possibly a slight centroid shift to the NE with decreasing surface brightness.

Figure 16 quantifies this by plotting the locations of the fitted ellipse centers for successive contours. The ellipse centroids shift about 10 arcsec to the NE as we map fainter contours, consistent with the visual impression from Figure 15. This shift is quite modest: the centroid offsets in Figure 16 are never larger than about $5 \%$ of the King core radius of Leo I (IH95). Figure 17 plots some of the other parameters we derive from the fitted ellipse contours. Neither the position angles of the fitted ellipses nor their ellipticities vary more than 1-2 $\sigma$ about the observed means, though the trend in ellipticity (increasing outward) appears to be systematic. The properties of the contours in Figure 15 do not rule out weak tidal features in Leo I, but they do indicate that Leo I lacks prominent tidal distortions in its projected structure. Models of dSph galaxies that lack DM but are optimally aligned to conceal structural anomalies, still tend to exhibit larger centroid shifts and isophot variations than we observe in Leo I (Klessen and Kroupa 1998; Klessen and Zhao 2002; Fleck and Kuhn 2003; Read et al. 2006b).

\subsubsection{Tidal Imprints in Leo I: Kinematic Properties}

Tides should produce observable kinematic signatures, including large-scale streaming motions that mimic rotation (Piatek and Pryor 1995; Oh et al. 1995; Klessen and Zhao 2002) or that produce distinctive, rising dispersion profiles (Read et al. 2006b). This pseudorotation signal may be seen as a coherent velocity gradient across the galaxy roughly oriented along the projected orbital path. A persistent motivation for exploring dSph models that lack DM has been to determine if tidal effects alone can account for the observed dispersions and dispersion profiles. We have already shown that the mildly distorted structural properties of Leo I imply the galaxy contains a significant DM content. Our discussion here aims to explore the extent to which tides may have affected the kinematics of Leo I given the presence of a DM halo.

To search for streaming, we calculate the mean velocity differences, $\Delta v$, within of our entire sample of kinematic members of Leo I on either side of a bisector passing through the galaxy center (see Walker et al. 2006a). We repeat this for a range of bisectors, each oriented at different position angles, $\theta$, producing a function $\Delta v(\theta)$ (Figure 18, see Figure 22 for a graphical description of this procedure). When we apply this test to the full kinematic 
sample of Leo I members, we find a maximum $\Delta v$ of $1.8 \pm 1.2 \mathrm{~km} / \mathrm{s}$. Monte-Carlo simulations (described in detail below) indicate that this observed maximum value of $\Delta v$ occur by chance about $30 \%$ of the time when we consider all 328 Leo I kinematic members in our dataset. Thus, we find no compelling evidence for a velocity gradient in Leo I from the full kinematic sample or from the stars located within.

We can split the Leo I sample into two radial groups corresponding to an 'inner' subsample $(R \leq 400 \operatorname{arcsec} ; N=264)$ and an 'outer' subsample $(R>400 \operatorname{arcsec} ; N=64)$. The motivation for this division comes from Figure 19. All stars (members and nonmembers) in the inner subsample are uniformly distributed about the center of Leo I. In the outer subsample, non-members remain uniformly distributed, but the kinematic members appear elongated along a position angle of about 90/270 deg. This effect does not evidently result from any selection effect in our sample or fiber assignments.

The spatial differences of the inner/outer subsamples can also be illustrated by comparing the frequency of members and nonmembers as a function of position angle. Figure 20 shows that along a position angle of about $90 \pm 10 \mathrm{deg}$, a significantly higher proportion of members make up the full outer kinematic subsample. For the inner subsample, no such trend is seen. This behavior is evident from inspection in the spatial distribution of kinematic members and non-members plotted in Figure 1, more plainly illustrated in Figure 22 , We conclude that stars of the outer sample of Leo I follow a distribution that is elongated along an axis that is similar to, but possibly slightly offset from the axis corresponding to the maximum velocity gradient of Leo I members in the outer subsample.

We have applied the streaming test described above for Leo I members in both the inner and outer subsamples separately (Figure 18). The inner subsample alone still shows no convincing evidence of streaming. However, the further out we sample Leo I members, the stronger the streaming signal becomes in terms of $\Delta v$ (Figure 18) and, generally, significance (see below). Moreover, the bahavior of $\Delta v(\theta)$ is highly coherent, consistent with streaming motion along the $\mathrm{PA} \sim 90 / 270$ axis. We have fit the outer subsample (Leo I members only) to a linear velocity gradient model as a function of position angle. The strongest velocity gradient corresponds to $P A \sim 108 \pm 10 \mathrm{deg}$. The fitted slope is $-0.34 \pm 0.15 \mathrm{~km} / \mathrm{s} / \mathrm{arcmin}$ or $-0.0046 \pm 0.0020 \mathrm{~km} / \mathrm{s} / \mathrm{pc}$ (for $D=255 \mathrm{kpc}$ ). The sign of the gradient is such that outer subsample Leo I members to the west of the center of Leo I have on average positive velocities relative to the systemic velocity of the galaxy, while outer subsample members to the east have on average negative relative velocities (see Figure 22).

To determine the significance of this result, we ran Monte-Carlo simulations where we assigned the observed velocities to permutations of the stellar positions (each MC experiment consisted of 10000 trials). We then calculated $\Delta v$ as above for each simulated sample. The 
probabilities of seeing a value of $\Delta v$ as large or larger than the observed maximum $\Delta v$ at any position angle are given in Figure 18. The observed maximum value of $\Delta v$ is exceeded about $10 \%$ of the time for our simulations of the inner subsample (top panel, Figure 18), while for the outer subsamples (for $R>400,455$, and 600 arcsec), the probability of exceeding $\Delta v_{\max }$ is $(0.03,0.006,0.014)$, respectively. If we add the requirement that the simulations exhibit the coherence apparent in Figure 18, then virtually none of the simulations $(\leq 5$ out of 30000) for the three outer subsamples. We conclude that Leo I exhibits a statistically highly significant velocity gradient along an axis very close to its apparent major axis, but only among stars with projected radii $\geq 400$ arcsec. We shall refer to this radial distance at which the galaxy's kinematics change as the 'break radius', $R_{b}$, of Leo I.

\subsubsection{Tidal Imprints in Leo I: Population Segregation}

Our data also reveal evidence of a change in the stellar populations of Leo I at the break radius. To illustrate this, we have taken our photometry from Figure 2 and plotted it for the inner and outer subsample regions separately (Figure 23). Four regions in the CMD were identified that correspond to red giant branch stars (RGB; Region 1), asymptotic giant branch stars (AGB; Region 2), field stars (Region 3), and blue-loop stars (Region 4). Regions 1 and 2 correspond, roughly, to our kinematic selection region (see Figure 2). Table 7 lists the numbers of stars in each region from direct counts in the CMD, along with kinematic results for stars in each CMD region where available.

Consider first the RGB and AGB regions (Regions 1 and 2, respectively; the data used here are listed in Table 7). The ratio of RGB candidates in the inner and outer samples is $732 / 91=8.0$, and $72 / 18=4.0$ for the AGB candidates. If we only consider the kinematic sample and assign membership to stars based on their velocities, the RGB kinematic members in the inner and outer samples becomes 209/52 $=4.0$, while for the AGB stars the ratio is $42 / 1=42$. That is, the intermediate-age population traced by the AGB stars (Gallart et al. 1999a; Hernandez et al. 2000; Dolphin 2002) is almost exclusively located within with the break radius, $R_{b}=400$ arcsec. RGB stars, which arise from both the intermediate and older populations, extend over both the inner and outer regions.

This analysis underscores the value of the kinematic data to search for population gradients. Field stars, almost all certain non-members of Leo I based on their location in the CMD (Region 3 of Figure 23) ) exhibit a ratio of $\frac{N_{\text {outer }}}{N_{\text {inner }}}=2.1 \pm 0.5$, consistent with the ratio of the areas of the inner and outer regions $\left(\frac{A_{\text {outer }}}{A_{\text {inner }}}=2.6\right)$. This contamination explains the relatively small ratio of inner/outer AGB stars in the non-kinematic sample. Blue-loop stars (Region 4 in Figure 23) exhibit an inner/outer ratio of about 6.0, and, like the AGB, are 
intermediate-age stars (Dohm-Palmer and Skillman 2002). The actual ratio of inner/outer blue-loop stars is likely considerably larger since non-members preferentially contaminate the outer sample.

These points are presented graphically in Figure 24 where we plot cumulative radial distributions of various subsets of stars from the Leo I CMD (Figure 23). The left panel shows that, among radial-velocity members, stars selected in the AGB region of Figure 23 (Region 2) are more centrally distributed than the RGB stars (Region 1). This holds even if we only consider kinematically-selected RGB stars (which are biased in radius due to fiber restrictions and science aims), or the full sample of RGB candidates (thick and thin solid lines in Figure 24, respectively) noting that the counts of photometrically-selected RGB candidates are essentially complete at all radii. Virtually all (44 of 45) of the AGB members are inside $R_{b}$ despite the fact that we observed 10 stars from this region in the CMD outside the break radius. A KS test comparing the observed radial distributions of the AGB and RGB kinematic members reveals a low probability of $<0.01 \%$ that the two are drawn from a common parent distribution.

The right panel of Figure 24 addresses the possible segregation of AGB and blueloop stars from the older stars in Leo I and each other. The cumulative distribution of photometrically-selected AGB stars exhibits a change in slope near $R_{b}$. We know from the kinematic sample of AGB stars that this is where field contamination begins to dominate those counts. The blue-loop stars show a similar slope change, but at about $0.75 R_{b}$. If this break in slope is due to contamination by blue disk and halo stars, and background quasars and galaxies, the radial profile suggests that the blue-loop stars may be even more centrally concentrated than the AGB in Leo I. A KS test suggests that there is a $0.1 \%$ probability the radial profiles of the AGB and blue-loop photometric candidates are drawn from the same parent distribution, suggesting some difference in the distributions of these two populations.

We conclude that the Leo I stellar populations segregate by age such that the younger populations (AGB and blue-loop stars) are preferentially near the galaxy center relative to the older (RGB) population. Moreover, this segregation appears to occur at the break radius,

$R_{b}=400$ arcsec, where we see kinematic segregation (Figure 18) and (possibly) structural changes in Leo I (Figure 17).

\subsection{A Heuristic Model for Leo I}

We present here a descriptive model that aims to account for all the features we have identified in our study of Leo I, as well as many long-standing enigmas of this galaxy. We 
assume Leo I is bound to the Milky Way and is currently on a highly elliptical orbit; Figure13 shows a representative example. The period for the orbit in Figure 13 is about 5.5 Gyr; one orbit (defined here as one complete cycle in $R$ ) is shown in Figure 14. The other orbits that we consider in this discussion have similar periods. It is important to appreciate that these orbital periods are sufficiently long that it may not be valid to assume a static Milky Way potential or that Leo I has remained isolated from other objects in the halo. We begin our discussion considering only the effects of the last perigalactic passage of Leo I, about 1 Gyr ago for all cases considered here.

One aspect of an elliptical orbit that is well known (see King 1962; Allen and Richstone 1988; Read et al. 2006b; Choi et al. 2007) is that the instantaneous tidal radius of a dwarf galaxy in such an orbit varies with Galactocentric distance. When closest to the Galactic Center, for example, Leo I's true tidal radius (in the Roche sense) will be smallest, while far from the Galactic Center, the tidal radius will be large. For any assumed orbit, we can calculate the tidal radius crudely in a two-body approximation as $R_{t}=D\left(M_{L e o ~ I} / 2 M_{M W}\right)^{(1 / 3)}$ (we assume $M_{M W}>>M_{\text {Leo I }}$; King 1962). We can then ask, at what perigalactic distance does the minimum tidal radius, $R_{t, \min }$, equal the kinematic break radius, $R_{b}$, that we identified in Section 4.3.3? Figure 25 is a plot of $R_{t, \min }$ as a function of perigalacticon for orbits of Leo I in a logarithmic Milky Way potential with total mass (to the Virial radius) of $10^{12} M_{\odot}$ for a range of assumed Leo I mass, and for various assumed present-day Galactocentric tangential velocities for Leo I. Further details of these orbits are listed in Table 8. Our assumption that Leo I is bound to the Milky Way implies that the galaxy's present tangential velocity is less than about $100 \mathrm{~km} / \mathrm{s}$, so only results from models with $v_{\text {tan }} \leq 100 \mathrm{~km} / \mathrm{s}$ (Table 8) are plotted in Figure 25. The horizontal dashed line in Figure 25 corresponds to $R_{b}=500$ pc, equal to the physical size of the observed break radius $\left(R_{b}=400\right.$ arcsec $)$ at the current distance of Leo I.

For $M_{\text {Leo } I}=5 \times 10^{8} M_{\odot}$, we find that Leo I had to pass within about $4 \mathrm{kpc}$ from the Galactic Center for $R_{t, \min }=R_{b}$. Lower masses imply larger perigalactica, up to $\sim 20 \mathrm{kpc}$ for $M_{\text {Leo } I}=2 \times 10^{7} M_{\odot}$. The latter mass, the lowest considered in Figure 25, corresponds to a global mass-to-light ratio of $\sim 4$ for Leo I and is reasonable for a purely baryonic case. For a lower limit of $M / L=0.8$ (appropriate for the comparatively young central populations of Leo I; see M98s), we estimate that perigalacticon may have been as large as about $35 \mathrm{kpc}$.

One implication of this scenario is that many stars initially bound to Leo I prior to perigalactic passage, would have found themselves outside the galaxy's tidal radius as it passed by the Milky Way. Stars with radially-outbound orbits relative to the center of the dwarf would have begun to drift away at a relative speed comparable to the internal velocity dispersion, and in the process initiating the formation of tidal arms (Piatek and Pryor 1995; 
Oh et al. 1995; S07). Over the time interval, $t_{p}$, since perigalacticon, stars will drift a distance of order $\sigma t_{p}$ from the center of Leo I, or about $10-30 \mathrm{kpc}$ for $\sigma=10 \mathrm{~km} / \mathrm{s}$ for the orbits listed in Table 8. As Leo I continues to orbit away from the Milky Way, its tidal radius grows so that, at present, it is about $20 \mathrm{kpc}$. Consequently, some of the stars that were formally unbound at perigalacticon will be recaptured, but because the stars on rapid, radial orbits remain unbound, the velocity distribution will become progressively more tangentially anisotropic in the outer parts of the galaxy.

The key point is that for a satellite in an elliptical orbit, variations in $R_{t}$ will preferentially affect the kinematics of the outermost stars of the system, while leaving the kinematics internal to this radius comparatively unaffected (this just reiterates the conclusions of Piatek and Pryor 1995; Oh et al. 1995). We contend that our observation of a radius at which the internal kinematics of Leo I change suddenly may represent this incursion of $R_{t}$ into the main body of Leo I. This behavior is reflected in some of the models of Mayer et al. (2001a,b; 2005) though these authors did not explore orbits quite as eccentric as we are considering for Leo I.

It is reasonable to suppose that this simple model can plausibly account for the fairly mild structural anomalies we see in Leo I, including the small shift in its photocenter as a function of surface brightness and the elongation of the distribution of kinematic members at large radii. The timing of the last perigalactic passage is also very similar to the age of the end of the last prolonged burst in the star-formation history of Leo I (Gallart et al. 1999b; Hernandez et al. 2000; Dolphin 2002), while the spatial segregation of the stellar populations suggest that this event was largely confined to the inner regions of the galaxy. Hybrid $n$-body/hydro models (such as Mayer et al. 2005) predict that tidal stirring can result in a strong gaseous inflow in dwarf systems and centralized, bursty star formation, consistent with what we see in Leo I. The fact that population and/or chemical gradients are commonly seen in other local dwarfs (Harbeck et al. 2001; Tolstoy et al. 2004; Battaglia et al. 2006) suggests that tidal stirring could be a common process.

If we adopt $M / L \geq 0.8$ for Leo I and retain our interpretation of $R_{b}$ as $R_{t, \text { min }}$, then $R_{\text {apo }} \leq 400 \mathrm{kpc}$ for any reasonable Leo I mass and present-day tangential velocity. Defining the orbital eccentricity as $e=\left(1-R_{\text {peri }} / R_{\text {apo }}\right) /\left(1+R_{\text {peri }} / R_{\text {apo }}\right)$ for perigalactic and apogalactic distances $R_{\text {peri }}$ and $R_{\text {apo }}$, respectively, we conclude from the results in Table 8 and Figure 25 that $e \geq 0.74$ for $R_{\text {apo }}>255 \mathrm{kpc}$, the current distance of Leo I. For $M / L=20$, $R_{\text {peri }}=9 \mathrm{kpc}$ and $e \geq 0.93$. To the extent that equilibrium models are still valid near the core of Leo I (Read et al. 2006b; Klimentowsky et al. 2007), we can carry out a classic 'core fitting' analysis (Richstone and Tremaine 1986; M98) using the dispersion of only the inner subsample of Leo I members. From this we derive $M_{\text {Leo } I}=3.0 \times 10^{7} M_{\odot} \times\left(\sigma_{0} / 9.2\right)^{2}$, and 
infer $R_{\text {peri }} \sim 18 \mathrm{kpc}$ (Figure 25) and $e \geq 0.87$. These large eccentricities are consistent with the lack of strong spatial distortions in Leo I (Section 4.3.2; IH95) since it implies we are looking almost directly along the projected orbital path of the galaxy. If our interpretation of the fundamental physical origin of $R_{b}$ is correct, it is difficult to avoid the conclusion that Leo I passed very close to the Galactic center about 1 Gyr ago, regardless of its dark matter content.

This tidal history of Leo I implies that stars with positive velocities relative to the center of Leo I are in its leading (western) arm, while those with negative relative velocities are trailing (to the east). S07, whose model is broadly similar to the one we propose, came to the opposite conclusion. This appears to reflect the fact that the S07 $n$-body models include the results of two perigalactic passages. Since we consider only the effects of the last perigalactic passage in our description, we ignore stars that may be projected onto Leo I from portions of its orbit extremely far ahead or behind the main body of the galaxy. It may be that these stars - the ones from the perigalacticon some 7-9 Gyr ago - are the ones that contribute to the kinematic asymmetry S07 identify and interpret in their dataset.

S07 adopt a static Milky Way potential during the entire timespan between the present epoch and the last two perigalactica of Leo I (7-9 Gyr ago). It is conceivable that the Milky Way's gravitational potential may have changed significantly over that timespan (Bullock et al. 2001; Taylor and Babul 2004, 2005; Bell et al. 2006; though see Hammer et al. 2007) undermining the reliability of models in which Leo I orbits in a static potential (e.g. S07). On the other hand, we have so far adopted the seemingly ad hoc assumption that Leo I has had only one perigalactic passage with the Milky Way. This implies that Leo I was somehow injected into its present orbit sometime between the time of its last perigalactic passage (about 1 Gyr ago) and the time of its earlier putative perigalacticon 7-9 Gyr ago. Is this plausible?

There are two aspects to this question: First, is such an orbital change - presumably the result of an interaction with a third body - reasonably probable? If so, could such an interaction alter Leo I's orbit significantly without destroying the galaxy? For our purposes, 'significant' implies any change that causes Leo I to transition from an orbit with $R_{\text {peri }} \geq 50$ $\mathrm{kpc}$, to one that brings it in as close as $10 \mathrm{kpc}$ or so from the Galactic Center. From the data in Table 8, we estimate that the required change of orbital energy is as large as about 6\%. In the impulse approximation (Binney and Tremaine 1987), the energy change is approximately $\Delta E \sim G M_{p} / b$, for a perturber of mass $M_{p}$ and an encounter impact parameters $b$. Solving for $b$ and taking $\Delta E$ to be $6 \%$ of the total energy of Leo $I$ for an orbit that gives it $\left(v_{r}, v_{t}\right)=(180,80) \mathrm{km} / \mathrm{s}$ at $R=250 \mathrm{kpc}$ (Table 8 ), we find that for $M_{p}=$ $\left(10^{8}, 10^{9}, 10^{10}\right) M_{\odot}, b \sim(0.15,1.5,15) \mathrm{kpc}$ assuming that the energy change goes entirely into 
altering Leo I's orbit. If we consider a population of $N$ subhalos within the volume of the Milky Way's overall halo $\left(R_{M W} \sim 250 \mathrm{kpc}\right)$, then the instantaneous filling factor of those subhalos is $f=N\left(R_{s h} / R_{M W}\right)^{3}$. For the intermediate case above $\left(M_{p}=10^{9} M_{\odot}\right.$ for which $R_{s h} \sim b=1.5 \mathrm{kpc}$ ) and assuming $N=100, f \sim 9 \times 10^{-6}$ implying a very low probability of interaction.

But this calculation may be misleading. In hierarchical models, subhalos necessarily inhabit regions of comparatively high density right from the start, so they invariably have considerably more neighbors than in a uniform-density model. Moreover, the interactions may be 'slow' (relative velocities between subhalos comparable to the circular velocities of individual subhalos), contrary to a basic assumption of the impulse approximation where the relative velocity is taken to be comparable to the (large) dispersion of the overall halo in which the subhalos reside. Indeed, subhalo interactions are a common feature in CDM simulations of hierarchical structure formation (see web sites for: The Center of Theoretical Physics, Univ. of Zurich; The Center for Cosmological Physics, Univ. of Chicago; The $n$ body Shop, Univ. of Washington, Seattle). Qualitative inspection of these simulations seem to reveal that subhalos with large outward velocities become increasingly common, and that some of these cases are due to interactions with other subhalos and not the most central, parent halo.

Taylor and Babul (2004, 2005) explored this more quantitatively and confirmed work by Tormen et al. (1998) and Knebe et al. (2004) that showed that 'significant' encounters between subhalos are indeed quite common in systems forming hierarchically. In about $5 \%$ of the subhalo encounters, these interactions are transformative, in the sense that either or both halos disrupt or they merge together (Taylor and Babul 2004, 2005). But there is also a class of much weaker interactions that alter orbits but not raise destructive tides $(x>1$ in the nomenclature of Taylor and Babul, 2005, where $x$ is the ratio $b / r_{c, p}$, and $r_{c, p}$ is the radius of the peak of the rotation curve of a given subhalo). These are much more common than the transformative encounters, occurring at least once for 30-60\% of all subhalos during the formation of a Milky Way-sized galaxy. Most of these encounters (about 70\%; see Figure 19 of Taylor and Babul, 2005) occur over the first half of the formation process of a massive galaxy, compatible with our requirement that a third-body encounter altered Leo I's orbit up to 7-9 Gyr ago. More recently, Sales et al. (2007b) have confirmed these basic results from independent hierarchical models. They speculate that objects such as Leo I and some other odd Local Group galaxies (Cetus, Tucana) may have experienced third-body encounters that could account for their unusual orbital characteristics.

Zhao (1998) proposed a specific interaction between the Sgr dSph galaxy and the Magellanic Clouds 2-3 Gyr ago to inject Sgr into its current orbit. This model provides an elegant 
solution to the puzzle of the long-term survivability of Sgr (Zhao 1998; Bellazzini et al. 2006), and is consistent with its detailed orbital characteristics (Majewski et al. 2004). Perhaps something similar has occurred to Leo I, boosting the star formation rate and initiating its transformation into a spheroidal system (Mayer et al. 2001a,b) 7-9 Gyr ago while simultaneously injecting it into its present orbit. This picture also addresses Leo I's survival: two close encounters with the Milky Way should have stripped most of its initial mass (Mayer et al. 2001a,b; Taylor and Babul 2004, 2005; Read and Gilmore 2005), making it difficult for the galaxy to have survived as relatively unscathed as we observe today.

Detailed photometric studies of Leo I reveal low-level star formation for the first third of the galaxy's existence (Gallart et al. 1999b; Hernandez et al. 2000; Dolphin 2002) when Leo I may have resembled dIrr system. This was followed by an increase in star formation some 5-8 Gyr ago which ended, possibly after a final peak, about 1 Gyr ago. The event that first caused the star formation rate to rise evidently did not clear the gas from Leo I since star formation continued after that epoch. Nor did the event induce tidal features that we can see today in the distribution of stars in and around Leo I or in its internal kinematics. If it had, such features would by now extend over a very long arc of Leo I's present orbit and be seen as obvious photometric depth that is not observed (Section 4.3.2; Held et al. 2001). In our model, we identify this event as an interaction with another subhalo, specifically not with the Galactic Center. We obviously require a better idea of the orbit of Leo I before we can hope to identify the third body that Leo I may have scattered off of, assuming of course that that body exists as an identifiable entity today (Taylor and Babul 2004, 2005; Sales et al. 2007b).

The kinematic basis of the S07 model is their detection of a strong asymmetry in the velocity distribution of Leo I. We do not confirm this feature in our data. The velocity distribution of all Leo I members (Figure 26, $N=328$ ) has a skew of $0.08 \pm 0.14$ and a kurtosis of $-0.34 \pm 0.27$, both consistent with a Gaussian distribution. If we select stars from our sample that are spatially distributed in the same manner as those in the S07 sample (dashed histogram in Figure 26), we find no significant skew or kurtosis $(0.10 \pm 0.21$ and $-0.23 \pm 0.41$, respectively). It is unclear to what extent the systematic errors of the S07 velocities relative to our measurements (see Section 3.2) contribute to their observation of an asymmetric velocity distribution. What is clear is that we see no such effect in our larger sample of more precise velocities. In the detailed $n$-body models by S07, the asymmetry of the velocity distribution from their model appears to result entirely from stars that have migrated along the orbit since the penultimate perigalacticon (about 5-7 Gyr). Based on S07's reasoning, our data (Figure 26) argue that this earlier perigalactic passage did not occur and adds weight to the idea that Leo I's orbit has evolved. 
The extent of Leo I along the line of sight offers another way of distinguishing these models. S07 predict that Leo I should exhibit a full depth of about $30 \%$, defined here as $\frac{\Delta D}{D}$. In our description, this extent is much less, about $10-15 \%$, which we estimate from the distance stars have traveled relative to the center of Leo I at a velocity of 10 $\mathrm{km} / \mathrm{s}$ (characteristic of the internal dispersion) since perigalacticon. Proper simulations are needed to determine the tidal extent reliably, but we note that this rough estimate agrees with distance range of stars extracted from Leo I during its last perigalactic passage in the S07 models. Though the horizontal branch of Leo I is extended in luminosity, this seems to result mostly from its unusual star-formation history and not a distance spread (Gallart et al. 1999b). As we summarized in Section 4.3.2, observations of RR Lyr stars in Leo I appear to rule out a depth greater than about 15\% (Held et al. 2001), arguing against the existence of significant tidal arms as predicted by S07. Distance tracers accurate to $5 \%$ are needed if we hope to detect the much more modest tidal arms hypothesized in our scenario.

We predict that Leo I's proper motion should be from east to west in a heliocentric reference frame; specific predictions are given in Table 8. These values differ significantly from the predicted proper motion reported by S07 for several reasons. First, S07 report their prediction in units of marcsec/yr, though the values appear more consistent with units of $\operatorname{arcsec} / y r$. Also, the predicted proper motion in S07 is stated to be heliocentric, but the signs of the two components are inconsistent with this and suggest that the values reported by S07 correspond to a Galactostationary frame. Note that our predicted proper motions assume the orbital pole given by S07 and rely on our interpretation that the leading side of Leo I (west) exhibits a net positive velocity relative to the systemic velocity of the galaxy (see Figures 21 and 22).

One final issue has to do with why we see streaming in the outer parts of Leo I (outside the break radius, $R_{b}=400$ arcsec) while Koch et al. (2007; K07) do not. To explore this, we carried out simulations in which the velocities of stars in our sample were assumed to have (normal) errors 2.0 times as large as in Table 5, mimicking the mean uncertainty of the K07 measurements. For our full dataset, only $29 \%$ of our simulations produce by chance a larger value of $\Delta v$ than we observe (Figure 18). When we select stars to approximate the spatial distribution of stars in the K07 sample, $30 \%$ of the simulations produce a stronger streaming signal by chance. Interestingly, our data still reveal streaming outside $R_{b} \sim 400$ arcsec even when we double the velocity uncertainties or mimic the K07 spatial distribution. It will be of useful to expand the size of the Leo I kinematic sample, particularly at and outside $R_{b}$, to explore the nature of the streaming signal in Leo I further. 


\subsection{Halo Substructure?}

Figure 9 reveals the presence of some stars with velocities in the fairly narrow range 88 to $105 \mathrm{~km} / \mathrm{s}$. The mean heliocentric velocity of this group (14 measurements of 6 stars) is $95.8 \pm 2.4 \mathrm{~km} / \mathrm{s}$ with a 'dispersion' of $5.8 \pm 1.9 \mathrm{~km} / \mathrm{s}$. These stars appear uniformly distributed over the field and are kinematically distinct from Leo I. The expected distribution of field stars predicted by the Besancon Galaxy model (Robin et al. 2003) toward Leo I is shown in the Gaussian-smoothed histogram in the right panel of Figure 9. This model agrees reasonably well with the distribution of velocities of other non-Leo I stars plotted in Figure 9 and does very well in other dSph fields (Walker et al. 2007b). By counting stars in velocity intevals $<85 \mathrm{~km} / \mathrm{s}$ and between $85-110 \mathrm{~km} / \mathrm{s}$ in both our dataset and the model, we find that these 6 stars represent a modest $\sim 2 \sigma$ excess relative to this model, which predicts $1.2 \pm 0.2$ stars in this velocity interval given the number of field stars we observe at lower velocities. Other kinematic studies based on relatively high-resolution observations have identified possible evidence of cold kinematic groups in other halo fields (Cote et al 1992; Ibata et al. 1994; Odenkirchen et al. 2002; Muñoz et al. 2006). Given the complex and rich distribution of streams being discovered in wide-field surveys (Belakurov et al. 2006; Grillmair and Dionatos 2006), serendipitous kinematic detection of streams may not be surprising. We may be seeing a similar feature near Leo I, but the statistics are obviously poor and further members of this putative kinematic group need to be identified.

\section{Summary and Conclusions}

We have presented new kinematic results of stars located in and near the Milky Way satellite dwarf spheroidal galaxy Leo I. Our sample includes velocities of 328 likely Leo I red giant members based on new observations with the Hectochelle multi-object echelle spectrograph, plus published kinematic results obtained by M98 obtained with HIRES at the Keck Observatory. These results are based on measurements of spectra obtained around $5180 \AA$, a region virtually uncontaminated by telluric emission or absorption features.

Repeat measurements of many stars in our sample allow us to estimate the typical errors of the velocities of these stars to be $2.4 \mathrm{~km} / \mathrm{s}$. Our results give a systemic heliocentric velocity for Leo I of $282.9 \pm 0.5 \mathrm{~km} / \mathrm{s}$, and a radial velocity dispersion for the full sample of $9.2 \pm 0.4 \mathrm{~km} / \mathrm{s}$, both in agreement with previous measurements. The large areal coverage and significant number of stars in the present sample (see Figure 9) allow us to measure the radial velocity dispersion profile to slightly beyond the formal King tidal radius of Leo I (IH95; Table 6). As we find in other dSph systems (Walker et al. 2007b), this profile is flat to large projected radius (see Figures 10 and 12). 
We have fit the dispersion profile to a variety of equilibrium dynamical models. The observed profile is strongly inconsistent with an isotropic King model in which mass follows light, but can be fit reasonably well with an isothermal sphere. In this latter case, we still infer that the mass distribution is much more extended than the visible light. We have also fit the dispersion profile two-component Sersic+NFW model (Łokas 2002). The isothermal model implies a mass of $5.2 \pm 1.2 \times 10^{7} M_{\odot}$ within a radius of $1040 \mathrm{pc}$ and a central density of $\rho_{0}=0.23 \pm 0.04 M_{\odot} \mathrm{pc}^{-3}$ for a core radius equal to the King core radius. The best fit to a Sersic + NFW model gives a total mass of $7 \pm 1 \times 10^{8} M_{\odot}$ to a virial radius of $18.3 \mathrm{kpc}$ for $M_{t o t} / M_{v i s}=129 \pm 45$, and a tangentially anisotropic velocity distribution $(\beta=-1.5)$. An isotropic Sersic+NFW model can be excluded at $>95 \%$ confidence. These results are summarized in Table 6 and illustrated in Figure 12, All models that provide acceptable fits to the dispersion profile of Leo I demand that the DM profile is much more extended than the visible matter.

One motivation to study Leo I has been to determine the characteristics of a plausibly isolated dark halo. Ironically, our observations reveal evidence that tidal effects may actually have significantly affected the properties of the galaxy. We find evidence of a 'break' radius, $R_{b}$, at about $R=400 \operatorname{arcsec}(500 \mathrm{pc})$, where the internal kinematics of Leo I change from apparently isotropic inside this radius, to a distribution consistent with rotation or streaming along the major axis. Monte-Carlo simulations reveal that the statistical significance of the kinematic change is high $(>97 \%)$. We interpret this in the framework of a heuristic model in which Leo I passed very close to the center of the Milky Way about 1 Gyr ago. The break radius corresponds to the instantaneous tidal radius of Leo I at perigalacticon (Table 8 and Figure 25]).

This simple model accounts for the observed kinematic and population segregation in Leo I, the mildly distorted structural properties of the galaxy, the age and duration of the last prominent burst of star formation, and the large outward radial velocity of Leo I relative to the Galactic Center. The lack of gas today in the galaxy presumably reflects the fact that the ISM was largely consumed by star formation in the inner part of Leo I (inside $R_{b}$ ), and stripped via ram pressure near the Galactic disk outside that radius. This is consistent with our detection of population segregation in Leo I, such that younger stars predominate inside $R_{b}$, while older populations are found at all radii.

Tidal arms would have formed in Leo I during its perigalactic passage(s). Because of the high ellipticity of the galaxy's orbit and its large distance, we predict that these arms are projected close to the main body of the galaxy, and that they exhibit a full extent of about $10-15 \%$ the distance to Leo I $(25-35 \mathrm{kpc})$. In this picture, the leading (more distant) arm is associated with stars with positive velocities relative to the center of Leo I, corresponding 
to the west side of the galaxy. Existing observations of RR Lyr stars in Leo I appear to rule out arms that extend $20-30 \%$ of the distance to the galaxy along the line of sight (Held et al. 2001). Shoerter arms cannot be excluded with existing observations, but could be detectable with a distance indicator capable of distance resolution of $\sim 5 \%$, such as dwarf Cepheids (Mateo et al. 1998b).

The lack of long tidal arms is inconsistent with the simulations of S07 who argue Leo I has suffered at least two perigalactic passages in its lifetime. We speculate that Leo I may have instead been injected into its highly elliptical orbit via an interaction with a third body, similar to a more specific model in which Sgr was injected into its present orbit after interacting with the LMC (Zhao 1998). Within the context of hierarchical models, such an interaction for Leo I is not only possible, but probable (Taylor and Babul 2005, 2005; Sales et al. 2007b). This scattering event had to have occurred between about 2-9 Gyr ago to exclude as second close perigalactic passage of Leo I. The lack of asymmetry in the velocity distribution of our kinematic sample of Leo I members (Figure 26) is also consistent with only one close perigalactic passage during Leo I's lifetime (S07).

It would be of interest to look for a similar effect in other satellite systems. Our kinematic observations of nearly 1000 stars in Carina suggest that we may see evidence of a break radius in that galaxy at comparable significance (97\% ; Walker et al. 2007c). Other dwarfs for which we have extensive kinematic data also show possible break radii, but at lower significance than in Leo I. This may not be entirely surprising. Leo I's exceptionally large radial velocity makes the galaxy unique, and demands a highly elliptical orbit if it is bound to the Milky Way (Byrd et al. 1994; Taylor et al. 2005). Most other dSph systems appear to be on less extreme elliptical orbits (Piatek et al. 2005, 2006, 2007; Dinescu et al. 2004), so the break radius may not be as well-defined as in Leo I. Carina may have the next most extreme orbital eccentricity after Leo I ( $e_{C a r}=0.67$; Piatek et al. 2003). It has long been discussed as a system with an extended pseudo-stream of stars (Majewski et al. 2000; Muñoz et al. 2006), and we may have already detected a break radius in its kinematics. Carina contains dwarf Cepheids (e.g. Mateo et al. 1998b) which, if identified in greater numbers and over a larger fraction of the galaxy, could be used to explore the galaxy's line-of-sight extent.

Finally, it is worth noting here that the possibility that Leo I has been affected significantly by tides does not necessarily contradict our conclusion from equilibrium models that the system is dominated by dark matter. For the larger masses we derive from the NFW models, Leo I would have had to pass within $10 \mathrm{kpc}$ or so of the Galactic Center. Indeed, as long as we adopt Newtonian gravity, its survival in this orbit demands the existence of dark matter. If Leo I has no dark matter at all, then it could not have passed closer than about $40 \mathrm{kpc}$ of the Galactic Center and survive. Such a large perigalacticon may be prob- 
lematic for models that account for the gas loss via ram-pressure stripping and consumption in tidally-induced star formation (Mayer et al. 2001a,b, 2005). Measurements of precise proper motions can help settle this issue, though Table 8 suggests it may prove difficult to make fine distinctions within a broad range of plausible Leo I orbits. In the meantime, our observations suggest some obvious $n$-body simulations that could be designed to recreate

our detailed observations of the dispersion profile and break radius in the enigmatic dwarf galaxy, Leo I (Klimentowski et al. 2007).

We thank Carlton Pryor for calculating a range of orbits for Leo I. Nelson Caldwell, Gabor Furesz and John Roll helped immensely in planning, scheduling and implementing our MMT observations, and we are grateful for their efforts. Andy Szentgyorgyi and Dan Fabricant helped to address occasional problems with Hectochelle promptly and expertly. We are grateful to the entire Hectochelle team for their impressive efforts in building and supporting this complex instrument. At the telescope, we were expertly helped by Hectochelle robot operators, Perry Berlind and Michael Calkins, and the MMT operators Mike Alegria, John McAfee, and Alejandra Milone. We thank them for ensuring that our runs were successful and pleasureable. We are grateful to the referee for an insightful and thorough report. This work has been supported by NSF grants AST 02-06081 and AST 05-07453 (to MM) and AST 02-05790 and AST 05-07511 (to EO).

\section{REFERENCES}

Allen, A. J., \& Richstone, D. O. 1988, ApJ, 325, 583

Battaglia, G., et al. 2006, A\&Ap, 459, 423

Bekenstein, J. D. 2004, Phys. Rev. D, 70, 083509

Bell, E. F., Phleps, S., Somerville, R. S., Wolf, C., Borch, A., \& Meisenheimer, K. 2006, ApJ, 652, 270

Bellazzini, M., Correnti, M., Ferraro, F. R., Monaco, L., \& Montegriffo, P. 2006, A\&A, 446, L1

Bellazzini, M., Gennari, N., Ferraro, F. R., \& Sollima, A. 2004, MNRAS, 354, 708

Belokurov, V. et al. 2006, ApJ, 642, L137

Benson, A. J., Frenk, C. S., Lacey, C. G., Baugh, C. M., \& Cole, S. 2002, MNRAS, 333, 177

Binney, J., \& Tremaine, S. 1987, Galactic Dynamics, Princeton University Press (Princeton, NJ) 
Brown, T. M., Smith, E., Guhathakurta, P., Rich, R. M., Ferguson, H. C., Renzini, A., Sweigart, A. V., \& Kimble, R. A. 2006, ApJ, 636, L89

Bullock, J. S., Kolatt, T. S., Sigad, Y., Somerville, R. S., Kravtsov, A. V., Klypin, A. A., Primack, J. R., \& Dekel, A. 2001, MNRAS, 321, 559

Byrd, G., Valtonen, M., McCall, M., Innanen, K. 1994, AJ, 107, 2055

Caputo, F., Cassisi, S., Castellani, M., Marconi, G., \& Santolamazza, P. 1999, AJ, 117, 2199

Choi, J.-H., Weinberg, M. D., \& Katz, N. 2007, ArXiv Astrophysics e-prints, arXiv:astro-ph/0702353

Coleman, M. G., Da Costa, G. S., Bland-Hawthorn, J., \& Freeman, K. C. 2005, AJ, 129, 1443

Coleman, M., Da Costa, G. S., Bland-Hawthorn, J., Martínez-Delgado, D., Freeman, K. C., \& Malin, D. 2004, AJ, 127, 832

Cote, P., Welch, D. L., Fischer, P., \& Irwin, M. J. 1993, ApJ, 406, L59

Connors, T. W., Kawata, D., \& Gibson, B. K. 2006, MNRAS, 371, 108

Demers, S., \& Kunkel, W. E. 1979, PASP, 91, 761

Demers, S., Battinelli, P., Irwin, M. J., \& Kunkel, W. E. 1995, MNRAS, 274, 491

Dinescu, D. I., Keeney, B. A., Majewski, S. R., \& Girard, T. M. 2004, AJ, 128, 687

Dohm-Palmer, R. C., \& Skillman, E. D. 2002, AJ, 123, 1433

Dolphin, A. E. 2002, MNRAS, 332, 91

Ferrara, A., \& Tolstoy, E. 2000, MNRAS, 313, 291

Fich, M., \& Tremaine, S. 1991, ARA\&A, 29, 409

Fleck, J.-J., \& Kuhn, J. R. 2003, ApJ, 592, 147

Gallart, C., Freedman, W.L., Mateo, M., Chiosi, C., Thompson, I.B., Aparicio, A., Bertelli, G., Hodge, P.W., Lee, M.G., Olszewski, E.W., Saha, A., Stetson, P.B., Suntzeff, N.B. 1999a, ApJ, 514, 665

Gallart, C., Freedman, W. L., Aparicio, A., Bertelli, G., \& Chiosi, C. 1999b, AJ, 118, 2245

Girardi, L., Bertelli, G., Bressan, A., Chiosi, C., Groenewegen, M. A. T., Marigo, P., Salasnich, B., \& Weiss, A. 2002, A\&A, 391, 195

Grebel, E. K., \& Gallagher, J. S., III 2004, ApJ, 610, L89

Grillmair, C. J. 2006, ApJ, 645, L37

Grillmair, C. J., \& Dionatos, O. 2006, ApJ, 643, L17 
Hammer, F., Puech, M., Chemin, L., Flores, H., \& Lehnert, M. 2007, ArXiv Astrophysics e-prints, arXiv:astro-ph/0702585

Harbeck, D., et al. 2001, AJ, 122, 3092

Hargreaves, J. C., Gilmore, G., \& Annan, J. D. 1996, MNRAS, 279, 108

Held, E. V., Clementini, G., Rizzi, L., Momany, Y., Saviane, I., \& Di Fabrizio, L. 2001, ApJ, 562, L39

Held, E. V., Saviane, I., Momany, Y., \& Carraro, G. 2000, ApJ, 530, L85

Hernandez, X., Gilmore, G., \& Valls-Gabaud, D. 2000, MNRAS, 317, 831

Ibata, R. A., Gilmore, G., \& Irwin, M. J. 1994, Nature, 370, 194

Ibata, R., Irwin, M., Lewis, G., Ferguson, A. M. N., \& Tanvir, N. 2001, Nature, 412, 49

Irwin, M., \& Hatzidimitriou, D. 1995, MNRAS, 277, 1354 (IH95)

Johnston, K. V., Spergel, D. N., \& Hernquist, L. 1995, ApJ, 451, 598

King, I. 1962, AJ, 67, 471

King, I. R. 1966, AJ, 71, 64

Klessen, R. S., Grebel, E. K., \& Harbeck, D. 2003, ApJ, 589, 798

Klessen, R. S., \& Kroupa, P. 1998, ApJ, 498, 143

Klessen, R. S., \& Zhao, H. 2002, ApJ, 566, 838

Kleyna, J. T., Wilkinson, M. I., Evans, N. W., \& Gilmore, G. 2004, MNRAS, 354, L66

Klimentowski, J., Łokas, E. L., Kazantzidis, S., Prada, F., Mayer, L., \& Mamon, G. A. 2007, MNRAS, 378, 353

Koch, A., Wilkinson, M. I., Kleyna, J. T., Gilmore, G. F., Grebel, E. K., Mackey, A. D., Evans, N. W., \& Wyse, R. F. G. 2007, ApJ, 657, 241 (K07)

Kuhn, J. R., \& Miller, R. H. 1989, ApJ, 341, L41

Kuhn, J. R., Smith, H. A., \& Hawley, S. L. 1996, ApJ, 469, L93

Kormendy, J., \& Kennicutt, R. C., Jr. 2004, ARAA, 42, 603

Knapp, G. R., Kerr, F. J., \& Bowers, P. F. 1978, AJ, 83, 360

Knebe, A., Gill, S. P. D., Gibson, B. K., Lewis, G. F., Ibata, R. A., \& Dopita, M. A. 2004, ApJ, 603, 7

Kochanek, C. S. 1996, ApJ, 457, 228

Kravtsov, A. V., Gnedin, O. Y., \& Klypin, A. A. 2004, ApJ, 609, 482 
Landolt, A. U. 1983, AJ, 88, 439

Landolt, A. U. 1992, AJ, 104, 340

Łokas, E. L. 2002, MNRAS, 333, 697

Majewski, S. R., et al. 2004, AJ, 128, 245

Majewski, S. R., Ostheimer, J. C., Patterson, R. J., Kunkel, W. E., Johnston, K. V., \& Geisler, D. 2000, AJ, 119, 760

Majewski, S. R., et al. 2005, AJ, 130, 2677

Majewski, S.R., Skrutskie, M.F., Weinberg, M.D., Ostheimer, J.C. 2003, ApJ, 599, 1082

Martínez-Delgado, D., Alonso-García, J., Aparicio, A., \& Gómez-Flechoso, M. A. 2001, ApJ, 549, L63

Mashchenko, S., Carignan, C., \& Bouchard, A. 2004, MNRAS, 352, 168

Mateo, M. 1998, AR\&A, 36, 435

Mateo, M., Olszewski, E.W., Vogt, S.S., Keane, M.J. 1998a, AJ, 116, 231 (M98)

Mateo, M., Hurley-Keller, D., Nemec, J. 1998b, AJ, 115, 1856

Mayer, L. 2005, IAUC 198, "Near-field Cosmology with Dwarf Elliptical Galaxies," eds. Jerjen \& Bingelli

Mayer, L., Governato, F., Colpi, M., Moore, B., Quinn, T., Wadsley, J., Stadel, J., Lake, G. 2001a, ApJ, 547, L123

Mayer, L., Governato, F., Colpi, M., Moore, B., Quinn, T., Wadsley, J., Stadel, J., Lake, G. 2001b, ApJ, 559, 754

Mayer, L., Kazantzidis, S., Mastropietro, C., \& Wadsley, J. 2007, Nature, 445, 738

Mayer, L., Mastropietro, C., Wadsley, J., Stadel, J., \& Moore, B. 2006, MNRAS, 369, 1021

Merritt, D., \& Saha, P. 1993, ApJ, 409, 75

Menzies, J., Feast, M., Tanabe, T., Whitelock, P., Nakada, Y. 2002, MNRAS, 335, 923

Metz, M., Kroupa, P., \& Jerjen, H. 2007, MNRAS, 374, 1125

Milgrom, M. 1983a, ApJ, 270, 371

Milgrom, M. 1983b, ApJ, 270, 384

Monelli, M., et al. 2004, Mem. della Soc. Astr. It. Supp., 5, 65

Monet, D. G., et al. 2003, AJ, 125, 984

Moore, B., Ghigna, S., Governato, F., Lake, G., Quinn, T., Stadel, J., \& Tozzi, P. 1999, ApJ, 524, L19 
Muñoz, R. R., et al. 2005, ApJ, 631, L137

Muñoz, R. R., et al. 2006, ApJ, 649, 201

Navarro, J. F., Frenk, C. S., \& White, S. D. M. 1997, ApJ, 490, 493

Nemec, J. M., Nemec, A. F. L., \& Lutz, T. E. 1994, AJ, 108, 222

Odenkirchen, M., Grebel, E. K., Dehnen, W., Rix, H.-W., \& Cudworth, K. M. 2002, AJ, 124,1497

Oh, K. S., Lin, D. N. C., \& Aarseth, S. J. 1995, ApJ, 442, 142

Olsen, K. A. G., \& Massey, P. 2007, ApJ, 656, L61

Olszewski, E. W., Mateo, M., Harris, J., Walker, M. G., Coleman, M. G., \& Da Costa, G. S. 2006, AJ, 131, 912

Olszewski, E. W., Pryor, C., \& Armandroff, T. E. 1996, AJ, 111, 750

Palma, C., Majewski, S. R., Siegel, M. H., Patterson, R. J., Ostheimer, J. C., \& Link, R. 2003, AJ, 125, 1352

Piatek, S., \& Pryor, C. 1995, AJ, 109, 1071

Piatek, S., Pryor, C., Olszewski, E. W., Harris, H. C., Mateo, M., Minniti, D., \& Tinney, C. G. 2003, AJ, 126, 2346

Piatek, S., Pryor, C., Bristow, P., Olszewski, E. W., Harris, H. C., Mateo, M., Minniti, D., \& Tinney, C. G. 2005, AJ, 130, 95

Piatek, S., Pryor, C., Bristow, P., Olszewski, E. W., Harris, H. C., Mateo, M., Minniti, D., \& Tinney, C. G. 2006, AJ, 131, 1445

Piatek, S., Pryor, C., Bristow, P., Olszewski, E. W., Harris, H. C., Mateo, M., Minniti, D., \& Tinney, C. G. 2007, AJ, 133, 818

Pichardo, B., Sparke, L. S., \& Aguilar, L. A. 2005, MNRAS, 359, 521

Putman, M. E., et al. 1998, Nature, 394, 752

Read, J. I. \& Gilmore, G. 2005, 356, 107

Read, J. I., Pontzen, A. P., \& Viel, M. 2006a, MNRAS, 371, 885

Read, J. I., Wilkinson, M. I., Evans, N. W., Gilmore, G., \& Kleyna, J. T. 2006b, MNRAS, 367,387

Rice, J. A., Mathematical Statistics and Data Analysis, 2nd ed. 1995, Wadsworth Publ. Co.

Richstone, D. O., \& Tremaine, S. 1986, AJ, 92, 72

Robin, A. C., Reylé, C., Derrière, S., \& Picaud, S. 2003, A\&Ap, 409, 523 
Sakamoto, T., Chiba, M., \& Beers, T. C. 2003, A\&A, 397, 899

Sales, L. V., Navarro, J. F., Abadi, M. G., \& Steinmetz, M. 2007a, ArXiv e-prints, 704, arXiv:0704.1770

Sales, L. V., Navarro, J. F., Abadi, M. G., \& Steinmetz, M. 2007b, ArXiv e-prints, 704, arXiv:0704.1773

Schechter, P. L., Mateo, M., \& Saha, A. 1993, PASP, 105, 1342

Sersic, J. L. 1968, Cordoba, Argentina: Observatorio Astronomico

Sohn, S. T., et al. 2007, ApJ, in press

Stefanik, R. P., Latham, D. W., \& Davis, R. J. 2006, PASP, 118, 1656

Sung, H., \& Bessell, M. S. 2000, Publications of the Astronomical Society of Australia, 17, 244

Susa, H., \& Umemura, M. 2004, ApJ, 600, 1

Szentgyorgyi, A. 2006, New Astronomy Review, 50, 326

Szentgyorgyi, A. H., Cheimets, P., Eng, R., Fabricant, D. G., Geary, J. C., Hartmann, L., Pieri, M. R., \& Roll, J. B. 1998, Proc. SPIE, 3355, 242

Taylor, J. E., \& Babul, A. 2004, MNRAS, 348, 811

Taylor, J. E., \& Babul, A. 2005, MNRAS, 364, 515

Taylor, J. E., Silk, J., \& Babul, A. 2005, IAU Colloq. 198: Near-fields cosmology with dwarf elliptical galaxies, 185

Tolstoy, E., et al. 2004, ApJ, 617, L119

Tonry, J., \& Davis, M. 1979, AJ, 84, 1511

Tormen, G, Diaferio, A., \& Syer, D. 1998, MNRAS, 299, 728

Udry, S., et al. 1999, ASP Conf. Ser. 185: IAU Colloq. 170: Precise Stellar Radial Velocities, 185,383

van den Bergh, S. 1994, ApJ, 428, 617

Walker, M. G., Mateo, M., Olszewski, E. W., Bernstein, R., Wang, X., \& Woodroofe, M. 2006a, AJ, 131, 2114

Walker, M. G., Mateo, M., Olszewski, E. W., Pal, J. K., Sen, B., \& Woodroofe, M. 2006b, ApJ, 642, L41

Walker, M. G., Mateo, M., Olszewski, E. W., Bernstein, R., Sen, B., \& Woodroofe, M. 2007a, ApJS, in press 
Walker, M. G., Mateo, M., Olszewski, E. W., Gnedin, O., Wang, X., Sen, B., \& Woodroofe, M. 2007b, ApJL, submitted

Walker, M. G., Mateo, M., \& Olszewski, E. W. 2007c, in preparation

Wang, X., Woodroofe, M., Walker, M. G., Mateo, M., \& Olszewski, E. 2005, ApJ, 626, 145

Wilkinson, M. I., \& Evans, N. W. 1999, MNRAS, 310, 645

Williams, G. G.,Olszewski, E., Lesser, M. P., \& Burge,J. H. 2004, Proc. SPIE, 5492, 787

Young, L. M. 1999, AJ, 117, 1758

Zaritsky, D., Olszewski, E.W., Schommer, R.A., Peterson, R.C., Aaronson, M. 1989, ApJ, 345,759

Zhao, H. 1998, ApJ, 500, L149 
Table 1: Log of Hectochelle Observations ${ }^{a}$

\begin{tabular}{lcccccc}
\hline Configuration & UT Date & UT Start/End & $\begin{array}{c}\text { ET } \\
(\mathrm{sec})\end{array}$ & $N_{\text {exp }}$ & $\alpha_{J 2000}$ & $\delta_{J 2000}$ \\
& & & & & \\
\hline SA57-n1 & Mar 31, 2005 & $08: 35 / 08: 46$ & 900 & 3 & $13: 05: 09.9$ & $+30: 06: 32$ \\
HD 171232 & Mar 31, 2005 & $12: 05 / 12: 11$ & 180 & 3 & $18: 32: 35.9$ & $+25: 32: 05$ \\
SA57-n3 & Apr 2, 2005 & $07: 34 / 07: 40$ & 600 & 2 & $13: 05: 09.9$ & $+30: 06: 32$ \\
SA57-2006 & Apr 19, 2006 & $08: 12 / 08: 28$ & 900 & 2 & $13: 05: 09.9$ & $+30: 06: 32$ \\
& & & & & & \\
Leo I/c1 & Mar 31, 2005 & $03: 03 / 07: 18$ & 16200 & 6 & $10: 08: 35.7$ & $+12: 16: 49$ \\
Leo I/c2 & Apr 2, 2005 & $02: 56 / 06: 13$ & 14400 & 4 & $10: 08: 15.5$ & $+12: 20: 57$ \\
Leo I/c3 & Apr 19, 2006 & $04: 57 / 07: 45$ & 10000 & 4 & $10: 08: 23.2$ & $+12: 21: 32$ \\
Leo I/c4 & Apr 20, 2006 & $04: 29 / 06: 35$ & 7500 & 3 & $10: 08: 40.0$ & $+12: 16: 29$ \\
Leo I/c5 & Apr 24, 2006 & $03: 34 / 05: 52$ & 8100 & 3 & $10: 08: 25.9$ & $+12: 18: 36$ \\
Leo I/c6 & Mar 12, 2007 & $06: 44 / 09: 00$ & 8100 & 3 & $10: 08: 24.5$ & $+12: 18: 24$ \\
Leo I/c7 & Apr 22, 2007 & $03: 53 / 06: 20$ & 8100 & 3 & $10: 08: 24.5$ & $+12: 18: 24$ \\
\hline
\end{tabular}

${ }^{a} \mathrm{ET}$ is the total exposure time. $N_{\text {exp }}$ is the number of individual exposures obtained for each target. 
Table 2: Summary Standard-Star Observations ${ }^{\mathrm{a}}$

\begin{tabular}{|c|c|c|c|c|c|c|c|c|c|}
\hline \multirow[t]{2}{*}{ Star $^{b}$} & \multirow[t]{2}{*}{$v_{o b s, n 1}$} & \multirow[t]{2}{*}{$v_{o b s, n 3}$} & \multirow[t]{2}{*}{$v_{o b s, 06}$} & \multirow[t]{2}{*}{$v_{o b s, 07}$} & \multirow[t]{2}{*}{$v_{h}$} & \multicolumn{4}{|c|}{$\Delta v=v_{o b s}-v_{h}$} \\
\hline & & & & & & n1 & n3 & 06 & 07 \\
\hline W22942 & -11.00 & -14.23 & -12.42 & $\cdots$ & -16.35 & 5.35 & 2.12 & 3.93 & . \\
\hline W23082 & -17.51 & -20.76 & $\ldots$ & $\ldots$ & -21.69 & 4.18 & 0.93 & $\ldots$ & $\ldots$ \\
\hline W23108 & -10.91 & -14.33 & -12.25 & $\ldots$ & -16.49 & 5.58 & 2.16 & 4.24 & $\ldots$ \\
\hline W23131 & -1.53 & -4.80 & -2.69 & $\ldots$ & -6.02 & 4.49 & 1.22 & 3.33 & $\ldots$ \\
\hline W23833 & -0.36 & -1.93 & -1.66 & $\ldots$ & -4.95 & 4.59 & 3.02 & 3.29 & $\ldots$ \\
\hline W23870 & $\ldots$ & -11.60 & -11.49 & $\ldots$ & -14.89 & $\ldots$ & 3.29 & 3.40 & $\ldots$ \\
\hline W23961 & -2.88 & -4.42 & -3.91 & $\ldots$ & -7.10 & 4.22 & 2.68 & 3.19 & $\ldots$ \\
\hline W24128 & 8.16 & 4.32 & 5.93 & $\ldots$ & 2.11 & 6.05 & 2.21 & 3.82 & $\ldots$ \\
\hline W24226 & 16.58 & 14.86 & . & $\ldots$ & 11.62 & 4.96 & 3.24 & ... & $\ldots$ \\
\hline W25209 & $\ldots$ & $\ldots$ & $\ldots$ & -13.35 & -16.73 & $\ldots$ & $\ldots$ & $\ldots$ & 3.38 \\
\hline W33245 & -16.36 & $\ldots$ & -17.68 & $\ldots$ & -20.97 & 4.61 & $\ldots$ & 3.29 & $\ldots$ \\
\hline W50001 & $\ldots$ & $\ldots$ & $\ldots$ & -68.71 & -71.91 & $\ldots$ & $\ldots$ & $\ldots$ & 3.20 \\
\hline W50325 & $\ldots$ & . . & . & 35.90 & 33.34 & $\ldots$ & $\ldots$ & . & 2.56 \\
\hline W50747 & $\ldots$ & $\ldots$ & . & 57.29 & 54.24 & $\ldots$ & $\ldots$ & . & 3.05 \\
\hline W50806 & $\ldots$ & $\ldots$ & . & -41.73 & -45.37 & . & $\ldots$ & . & 3.64 \\
\hline W50807 & $\ldots$ & $\ldots$ & $\ldots$ & 2.36 & -0.78 & $\ldots$ & $\ldots$ & $\ldots$ & 3.14 \\
\hline Average $^{c}$ & & & & & & 4.89 & 2.22 & 3.56 & 3.16 \\
\hline Corrected $^{\mathrm{c}}$ & & & & & & 1.49 & -1.18 & 0.16 & -0.24 \\
\hline Stand. Dev. & & & & & & 0.61 & 1.00 & 0.36 & 0.36 \\
\hline
\end{tabular}

${ }^{a}$ The subscripts $n 1$ and $n 3$ refer to results obtained on the first and third nights of our 2005 run. The subscripts '06' and '07' refer to SA57 observations during the 2006 and 2007 queue runs, respectively. See Section 2 and Table 1 for further details.

${ }^{b}$ Star designations and heliocentric velocities, $v_{h}$, are from Stefanik et al. (2006) for velocity standards near the North Galactic Pole (SA57).

c'Average' refers to the straight average $\Delta v$ for a given run/night. 'Corrected' refers to this average value minus $3.4 \mathrm{~km} / \mathrm{s}$ to account for the zero-point offset we found for our adopted template, HD171232 (see Section 3.1). 
Table 3: Statistics of Leo I Hectochelle Fiber Configurations ${ }^{\mathrm{a}}$

\begin{tabular}{|c|c|c|c|}
\hline Configuration & $N_{\text {assigned }}$ & $N_{2.8}$ & $N_{\text {LeoI }}$ \\
\hline c1/2005 & 109 & 82 & 54 \\
\hline $\mathrm{c} 2 / 2005$ & 99 & 78 & 58 \\
\hline c3/2006 & 105 & 87 & 71 \\
\hline c4/2006 & 115 & 65 & 51 \\
\hline c5/2006 & 107 & 68 & 57 \\
\hline c6/2007 & 114 & 72 & 63 \\
\hline c7/2007 & 100 & 91 & 86 \\
\hline Total/2005 & 208 & 160 & 112 \\
\hline Total/2006 & 327 & 220 & 179 \\
\hline Total/2007 & 214 & 163 & 149 \\
\hline Total & 749 & 543 & 440 \\
\hline Efficiency $/ 2005^{\mathrm{b}}$ & $\ldots$ & 0.77 & 0.54 \\
\hline Efficiency $/ 2006^{b}$ & ... & 0.67 & 0.55 \\
\hline Efficiency $/ 2007^{\mathrm{b}}$ & $\ldots$ & 0.76 & 0.70 \\
\hline Total Efficiency & $\ldots$ & 0.72 & 0.59 \\
\hline
\end{tabular}

${ }^{a}$ The counts in this table are, for each fiber configuration, the total number of fibers assigned to a target ( $\left.N_{\text {assigned }}\right)$, the total number of spectra that produce cross correlations with $R_{T D} \geq 2.8\left(N_{2.8}\right)$, and the number of Leo I velocity members $\left(N_{L e o I}\right.$, where membership is defined by whether a star has a heliocentric velocity in the range +250 to $+320 \mathrm{~km} / \mathrm{s}$; Section 3.2 and Figure 9). Configuration numbers are defined in Table 1.

${ }^{b}$ Efficiencies are defined as $N_{i} / N_{\text {assigned }}$, where $i$ refers to the various counts listed in this table. 
Table 4. Summary of Repeat Observations of Leo I Targets ${ }^{\mathrm{a}}$

\begin{tabular}{rccccccccc}
\hline \hline ID & $\begin{array}{c}v_{h, 1}, \sigma_{1} \\
(\mathrm{~km} / \mathrm{s})\end{array}$ & $\begin{array}{c}v_{h, 2}, \sigma_{2} \\
(\mathrm{~km} / \mathrm{s})\end{array}$ & $\begin{array}{c}v_{h, 3}, \sigma_{3} \\
(\mathrm{~km} / \mathrm{s})\end{array}$ & $\begin{array}{c}v_{h, 4}, \sigma_{4} \\
(\mathrm{~km} / \mathrm{s})\end{array}$ & $\begin{array}{c}v_{h, 5}, \sigma_{5} \\
(\mathrm{~km} / \mathrm{s})\end{array}$ & $\begin{array}{c}v_{h, 6}, \sigma_{6} \\
(\mathrm{~km} / \mathrm{s})\end{array}$ & $\begin{array}{c}v_{h, 7}, \sigma_{7} \\
(\mathrm{~km} / \mathrm{s})\end{array}$ & $\begin{array}{c}v_{h, 8}, \sigma_{8} \\
(\mathrm{~km} / \mathrm{s})\end{array}$ & $\begin{array}{c}v_{\text {avg }}, \sigma_{\text {avg }} \\
(\mathrm{km} / \mathrm{s})\end{array}$ \\
\hline 5 & $286.8,1.8$ & $287.8,1.7$ & $288.6,1.7$ & $286.3,1.7$ & $\ldots$ & $\ldots$ & $\ldots$ & $\ldots$ & $287.4,2.0$ \\
12 & $294.1,1.8$ & $\ldots$ & $\ldots$ & $298.9,1.8$ & $\ldots$ & $\ldots$ & $\ldots$ & $295.3,1.8$ & $295.9,2.8$ \\
16 & $\ldots$ & $\ldots$ & $\ldots$ & $\ldots$ & $284.7,1.8$ & $\ldots$ & $\ldots$ & $282.5,1.8$ & $283.6,2.1$ \\
23 & $\ldots$ & $281.4,1.9$ & $\ldots$ & $281.1,1.8$ & $\ldots$ & $\ldots$ & $\ldots$ & $\ldots$ & $281.2,1.9$ \\
25 & $\ldots$ & $287.1,1.7$ & $284.6,1.8$ & $283.9,1.9$ & $\ldots$ & $\ldots$ & $\ldots$ & $\ldots$ & $285.2,2.3$ \\
28 & $\ldots$ & $\ldots$ & $284.7,1.8$ & $282.9,1.9$ & $\ldots$ & $\ldots$ & $\ldots$ & $284.2,1.8$ & $283.9,2.0$ \\
31 & $287.4,1.8$ & $\ldots$ & $291.1,1.8$ & $\ldots$ & $\ldots$ & $\ldots$ & $\ldots$ & $289.4,1.8$ & $289.3,2.4$ \\
36 & $280.9,2.0$ & $280.4,1.9$ & $\ldots$ & $282.2,1.8$ & $\ldots$ & $\ldots$ & $\ldots$ & $282.6,1.8$ & $281.6,2.1$ \\
38 & $273.7,1.9$ & $275.8,1.8$ & $\ldots$ & $\ldots$ & $274.6,1.9$ & $\ldots$ & $\ldots$ & $276.8,1.8$ & $275.2,2.2$ \\
40 & $\ldots$ & $\ldots$ & $\ldots$ & $\ldots$ & $294.6,1.9$ & $\ldots$ & $\ldots$ & $305.8,1.8$ & $300.3,6.5$ \\
44 & $\ldots$ & $\ldots$ & $287.1,1.8$ & $\ldots$ & $285.5,1.8$ & $\ldots$ & $\ldots$ & $287.7,1.8$ & $286.8,2.0$ \\
47 & $280.0,1.9$ & $282.3,1.7$ & $\ldots$ & $280.3,1.9$ & $\ldots$ & $\ldots$ & $\ldots$ & $\ldots$ & $280.9,2.1$ \\
48 & $\ldots$ & $298.2,2.1$ & $\ldots$ & $\ldots$ & $300.3,2.0$ & $\ldots$ & $\ldots$ & $\ldots$ & $299.3,2.3$ \\
49 & $\ldots$ & $\ldots$ & $\ldots$ & $\ldots$ & $293.5,2.0$ & $\ldots$ & $\ldots$ & $289.6,1.8$ & $291.5,2.9$ \\
57 & $291.7,1.8$ & $291.5,1.9$ & $292.5,1.7$ & $293.1,1.7$ & $\ldots$ & $\ldots$ & $\ldots$ & $290.1,1.8$ & $291.8,2.1$ \\
62 & $\ldots$ & $311.5,1.8$ & $308.6,1.8$ & $307.3,1.8$ & $\ldots$ & $\ldots$ & $\ldots$ & $306.8,1.8$ & $308.3,2.6$ \\
65 & $296.1,1.9$ & $295.2,1.8$ & $299.4,1.7$ & $299.2,1.7$ & $\ldots$ & $\ldots$ & $\ldots$ & $\ldots$ & $297.6,2.7$ \\
66 & $\ldots$ & $\ldots$ & $\ldots$ & $285.9,1.8$ & $\ldots$ & $\ldots$ & $\ldots$ & $287.6,1.8$ & $286.8,2.0$ \\
68 & $312.8,1.9$ & $\ldots$ & $\ldots$ & $\ldots$ & $311.9,1.8$ & $\ldots$ & $\ldots$ & $306.3,1.8$ & $311.1,3.7$ \\
77 & $283.8,1.8$ & $\ldots$ & $\ldots$ & $279.7,1.7$ & $\ldots$ & $\ldots$ & $\ldots$ & $\ldots$ & $281.7,2.9$ \\
85 & $290.5,1.9$ & $\ldots$ & $\ldots$ & $\ldots$ & $\ldots$ & $\ldots$ & $\ldots$ & $294.3,1.8$ & $292.5,2.8$ \\
89 & $280.9,1.8$ & $281.3,1.8$ & $281.1,1.8$ & $279.6,1.7$ & $\ldots$ & $\ldots$ & $\ldots$ & $\ldots$ & $280.7,1.9$
\end{tabular}


Table 4-Continued

\begin{tabular}{|c|c|c|c|c|c|c|c|c|c|}
\hline ID & $\begin{array}{c}v_{h, 1}, \sigma_{1} \\
(\mathrm{~km} / \mathrm{s})\end{array}$ & $\begin{array}{c}v_{h, 2}, \sigma_{2} \\
(\mathrm{~km} / \mathrm{s})\end{array}$ & $\begin{array}{c}v_{h, 3}, \sigma_{3} \\
(\mathrm{~km} / \mathrm{s})\end{array}$ & $\begin{array}{c}v_{h, 4}, \sigma_{4} \\
(\mathrm{~km} / \mathrm{s})\end{array}$ & $\begin{array}{c}v_{h, 5}, \sigma_{5} \\
(\mathrm{~km} / \mathrm{s})\end{array}$ & $\begin{array}{c}v_{h, 6}, \sigma_{6} \\
(\mathrm{~km} / \mathrm{s})\end{array}$ & $\begin{array}{c}v_{h, 7}, \sigma_{7} \\
(\mathrm{~km} / \mathrm{s})\end{array}$ & $\begin{array}{c}v_{h, 8}, \sigma_{8} \\
(\mathrm{~km} / \mathrm{s})\end{array}$ & $\begin{array}{c}v_{a v g}, \sigma_{a v g} \\
(\mathrm{~km} / \mathrm{s})\end{array}$ \\
\hline 91 & & & $295.8,1.9$ & & $297.4,1.8$ & & & $293.4,1.8$ & $295.6,2.5$ \\
\hline 92 & $292.1,2.0$ & & $293.8,1.8$ & $293.8,1.8$ & $\ldots$ & 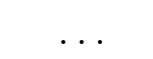 & $\ldots$ & 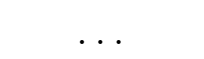 & $293.3,2.0$ \\
\hline 95 & $289.9,2.0$ & & $288.3,1.9$ & & $\ldots$ & $\ldots$ & $\ldots$ & $\ldots$ & $289.1,2.1$ \\
\hline 102 & 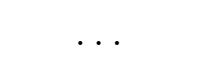 & $275.1,1.8$ & 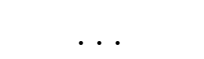 & $\ldots$ & $\ldots$ & $\ldots$ & $\ldots$ & $266.9,1.8$ & $271.0,4.9$ \\
\hline 107 & $299.4,1.9$ & $301.4,1.8$ & & $301.6,1.8$ & $\ldots$ & $\ldots$ & $\ldots$ & $\ldots$ & $300.8,2.1$ \\
\hline 109 & $291.4,2.0$ & $296.6,1.9$ & $298.8,1.9$ & & $\ldots$ & $\ldots$ & $\ldots$ & $\ldots$ & $296.2,3.9$ \\
\hline 113 & & & $286.9,1.9$ & & $287.8,1.9$ & $\ldots$ & $\ldots$ & $286.1,1.8$ & $286.9,2.0$ \\
\hline 116 & $274.4,2.0$ & $\ldots$ & $273.0,1.8$ & $272.7,1.8$ & $\ldots$ & $\ldots$ & $\ldots$ & $\ldots$ & $273.3,2.0$ \\
\hline 119 & $\ldots$ & $276.7,2.0$ & $274.4,2.0$ & $\ldots$ & $\ldots$ & $\ldots$ & $\ldots$ & $\ldots$ & $275.6,2.4$ \\
\hline 122 & & $283.9,1.9$ & $287.0,1.7$ & $\ldots$ & $\ldots$ & $\ldots$ & $\ldots$ & $\ldots$ & $285.5,2.5$ \\
\hline 124 & $282.6,1.8$ & & $276.5,1.6$ & $276.1,1.8$ & $\ldots$ & & $\ldots$ & $\ldots$ & $277.5,3.6$ \\
\hline 128 & $286.0,2.0$ & $288.3,1.9$ & $\ldots$ & $291.7,1.8$ & $\ldots$ & $\ldots$ & $\ldots$ & $\ldots$ & $288.6,3.2$ \\
\hline 135 & $\ldots$ & $277.9,1.9$ & $\cdots$ & $277.0,1.8$ & $278.9,1.9$ & $\ldots$ & $\ldots$ & $\ldots$ & $277.9,2.0$ \\
\hline 139 & $-8.2,1.7$ & $-4.1,1.7$ & $\ldots$ & $\ldots$ & $-4.6,1.8$ & $\ldots$ & $\ldots$ & $\ldots$ & $-5.4,2.6$ \\
\hline 150 & $-9.0,1.9$ & $-7.5,1.9$ & $-7.2,1.8$ & $-6.1,1.9$ & $\ldots$ & $\ldots$ & $\ldots$ & $\ldots$ & $-7.4,2.1$ \\
\hline 153 & $271.7,1.9$ & $272.2,1.9$ & & $265.9,1.8$ & $\ldots$ & $\ldots$ & $\ldots$ & & $270.7,3.7$ \\
\hline 157 & $276.7,1.9$ & $\ldots$ & $\ldots$ & $270.0,2.0$ & $\ldots$ & $\ldots$ & $\ldots$ & $\ldots$ & $273.4,4.3$ \\
\hline 161 & $284.1,1.8$ & $285.6,1.7$ & $280.7,1.7$ & $278.3,1.7$ & $\ldots$ & $\ldots$ & $\ldots$ & $\ldots$ & $282.3,3.7$ \\
\hline 170 & 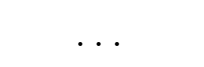 & $281.1,2.0$ & $285.9,1.9$ & $\ldots$ & $\ldots$ & $\ldots$ & $\ldots$ & $\ldots$ & $283.6,3.3$ \\
\hline 171 & & $296.6,2.0$ & & $301.1,2.1$ & $\ldots$ & $\ldots$ & $\ldots$ & $\ldots$ & $298.8,3.2$ \\
\hline 179 & $288.7,2.0$ & $290.6,1.8$ & $288.4,1.9$ & $291.2,2.0$ & 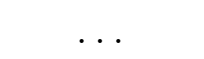 & $\ldots$ & $\ldots$ & $\ldots$ & $289.7,2.3$ \\
\hline 185 & $284.4,2.0$ & $286.0,1.9$ & $287.2,1.7$ & $\ldots$ & $\ldots$ & $\ldots$ & $287.2,1.7$ & $\ldots$ & $286.3,2.1$ \\
\hline
\end{tabular}


Table 4-Continued

\begin{tabular}{|c|c|c|c|c|c|c|c|c|c|}
\hline ID & $\begin{array}{l}v_{h, 1}, \sigma_{1} \\
(\mathrm{~km} / \mathrm{s})\end{array}$ & $\begin{array}{l}v_{h, 2}, \sigma_{2} \\
(\mathrm{~km} / \mathrm{s})\end{array}$ & $\begin{array}{c}v_{h, 3}, \sigma_{3} \\
(\mathrm{~km} / \mathrm{s})\end{array}$ & $\begin{array}{l}v_{h, 4}, \sigma_{4} \\
(\mathrm{~km} / \mathrm{s})\end{array}$ & $\begin{array}{l}v_{h, 5}, \sigma_{5} \\
(\mathrm{~km} / \mathrm{s})\end{array}$ & $\begin{array}{l}v_{h, 6}, \sigma_{6} \\
(\mathrm{~km} / \mathrm{s})\end{array}$ & $\begin{array}{l}v_{h, 7}, \sigma_{7} \\
(\mathrm{~km} / \mathrm{s})\end{array}$ & $\begin{array}{l}v_{h, 8}, \sigma_{8} \\
(\mathrm{~km} / \mathrm{s})\end{array}$ & $\begin{array}{c}v_{a v g}, \sigma_{a v g} \\
(\mathrm{~km} / \mathrm{s})\end{array}$ \\
\hline 192 & & & & & $300.4,2.0$ & $\ldots$ & $298.5,1.8$ & $\ldots$ & $299.4,2.1$ \\
\hline 195 & $279.7,2.1$ & $\ldots$ & $277.2,2.0$ & $272.0,2.0$ & $\ldots$ & $\ldots$ & $\ldots$ & $\ldots$ & $276.8,4.1$ \\
\hline 202 & $97.7,2.0$ & $99.2,2.0$ & $94.2,2.0$ & $\ldots$ & $\ldots$ & $\ldots$ & $\ldots$ & $\ldots$ & $97.2,3.0$ \\
\hline 213 & & $277.8,2.0$ & $285.0,1.8$ & $\ldots$ & $\ldots$ & $\ldots$ & $\ldots$ & $\ldots$ & $281.6,4.5$ \\
\hline 214 & $\ldots$ & $\ldots$ & $263.0,2.0$ & $259.9,2.0$ & $\ldots$ & $\ldots$ & $\ldots$ & $\ldots$ & $261.5,2.6$ \\
\hline 216 & $278.9,2.0$ & $\ldots$ & & $279.3,2.1$ & $\ldots$ & $\ldots$ & $\ldots$ & $\ldots$ & $279.1,2.0$ \\
\hline 221 & $272.9,2.1$ & $274.7,1.9$ & $272.6,1.7$ & $\ldots$ & $\ldots$ & $\ldots$ & $\ldots$ & $\ldots$ & $273.3,2.1$ \\
\hline 222 & 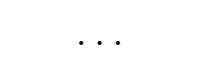 & $270.1,1.9$ & $265.2,1.7$ & $\ldots$ & $\ldots$ & $\ldots$ & $\ldots$ & $\ldots$ & $267.5,3.3$ \\
\hline 239 & $279.4,2.1$ & $280.9,1.9$ & $283.7,1.7$ & & $\ldots$ & $\ldots$ & $282.4,1.6$ & $\ldots$ & $281.7,2.5$ \\
\hline 244 & $89.0,2.0$ & $90.3,1.9$ & $\ldots$ & $\ldots$ & $\ldots$ & $\ldots$ & $\ldots$ & $\ldots$ & $89.7,2.0$ \\
\hline 248 & $35.8,2.0$ & $37.0,1.9$ & $34.8,1.7$ & $\ldots$ & $\ldots$ & $\ldots$ & $\ldots$ & $\ldots$ & $35.8,2.1$ \\
\hline 253 & & $290.3,1.8$ & $288.5,1.7$ & $289.0,1.7$ & $\ldots$ & $\ldots$ & $\ldots$ & $\ldots$ & $289.2,1.9$ \\
\hline 260 & & $296.2,1.9$ & & & $\ldots$ & $299.1,1.9$ & $\ldots$ & $\ldots$ & $297.7,2.5$ \\
\hline 262 & & $-12.7,1.9$ & $-12.8,1.7$ & & $\ldots$ & $\ldots$ & $\ldots$ & $\ldots$ & $-12.8,1.8$ \\
\hline 269 & & $299.4,2.0$ & $298.6,1.7$ & & $302.7,2.0$ & $\ldots$ & $\ldots$ & $\ldots$ & $300.0,2.6$ \\
\hline 272 & $289.7,2.0$ & & & $285.3,2.0$ & $\ldots$ & $\ldots$ & $285.4,1.7$ & $\ldots$ & $286.4,2.8$ \\
\hline 276 & $\ldots$ & $289.1,1.9$ & $291.3,1.7$ & $\ldots$ & $\ldots$ & $\ldots$ & $\ldots$ & $\ldots$ & $290.3,2.1$ \\
\hline 278 & $\ldots$ & $276.2,2.0$ & $277.8,1.9$ & $\ldots$ & $\ldots$ & $\ldots$ & $\ldots$ & $\ldots$ & $277.0,2.1$ \\
\hline 279 & $265.2,2.1$ & $267.4,1.9$ & $\ldots$ & $\cdots$ & $\ldots$ & $\ldots$ & $\ldots$ & $\ldots$ & $266.3,2.3$ \\
\hline 289 & $\ldots$ & $280.0,1.8$ & $281.7,1.8$ & $279.5,2.0$ & $\ldots$ & $\ldots$ & $\ldots$ & $\ldots$ & $280.4,2.1$ \\
\hline 295 & $\ldots$ & $309.2,1.9$ & $305.4,1.7$ & $\ldots$ & $\ldots$ & $\ldots$ & $\ldots$ & $\ldots$ & $307.2,2.8$ \\
\hline 302 & & & & $256.8,2.0$ & $\ldots$ & $264.9,2.0$ & $\ldots$ & $\ldots$ & $260.9,5.0$ \\
\hline
\end{tabular}


Table 4-Continued

\begin{tabular}{|c|c|c|c|c|c|c|c|c|c|}
\hline ID & $\begin{array}{c}v_{h, 1}, \sigma_{1} \\
(\mathrm{~km} / \mathrm{s})\end{array}$ & $\begin{array}{l}v_{h, 2}, \sigma_{2} \\
(\mathrm{~km} / \mathrm{s})\end{array}$ & $\begin{array}{c}v_{h, 3}, \sigma_{3} \\
(\mathrm{~km} / \mathrm{s})\end{array}$ & $\begin{array}{c}v_{h, 4}, \sigma_{4} \\
(\mathrm{~km} / \mathrm{s})\end{array}$ & $\begin{array}{c}v_{h, 5}, \sigma_{5} \\
(\mathrm{~km} / \mathrm{s})\end{array}$ & $\begin{array}{c}v_{h, 6}, \sigma_{6} \\
(\mathrm{~km} / \mathrm{s})\end{array}$ & $\begin{array}{l}v_{h, 7}, \sigma_{7} \\
(\mathrm{~km} / \mathrm{s})\end{array}$ & $\begin{array}{c}v_{h, 8}, \sigma_{8} \\
(\mathrm{~km} / \mathrm{s})\end{array}$ & $\begin{array}{c}v_{a v g}, \sigma_{a v g} \\
(\mathrm{~km} / \mathrm{s})\end{array}$ \\
\hline 308 & & $286.8,2.0$ & $287.0,2.0$ & & & & $\ldots$ & & $286.9,2.0$ \\
\hline 311 & $97.6,2.0$ & & & & $96.5,2.0$ & $\ldots$ & $\ldots$ & $\ldots$ & $97.0,2.1$ \\
\hline 312 & $\ldots$ & $281.3,2.0$ & $286.8,1.9$ & $\ldots$ & $\ldots$ & $\ldots$ & $\ldots$ & $\ldots$ & $284.1,3.7$ \\
\hline 313 & $\ldots$ & $275.4,2.1$ & $271.1,1.8$ & $\ldots$ & $\ldots$ & $\ldots$ & $\ldots$ & $\ldots$ & $273.1,3.1$ \\
\hline 319 & & & & $273.9,2.0$ & $\ldots$ & $273.7,2.0$ & $272.2,1.7$ & $\ldots$ & $273.2,2.0$ \\
\hline 322 & & & & & $301.7,2.0$ & $288.4,1.9$ & $\ldots$ & $\ldots$ & $295.2,7.6$ \\
\hline 324 & $280.5,1.8$ & $280.8,1.8$ & $285.0,1.8$ & $281.5,1.9$ & $\ldots$ & $\ldots$ & $\ldots$ & $\ldots$ & $281.7,2.6$ \\
\hline 333 & $\ldots$ & $264.9,2.1$ & & $267.6,2.1$ & $\ldots$ & $\ldots$ & $\ldots$ & $\ldots$ & $266.2,2.5$ \\
\hline 334 & $\ldots$ & $\ldots$ & $120.5,1.9$ & $119.9,2.0$ & $\ldots$ & $\ldots$ & $\ldots$ & $\ldots$ & $120.2,2.0$ \\
\hline 335 & $26.4,2.1$ & $16.9,2.0$ & & $\ldots$ & $\ldots$ & $\ldots$ & $\ldots$ & $\ldots$ & $21.6,5.7$ \\
\hline 336 & $270.6,2.1$ & $273.5,1.9$ & & & $\ldots$ & $\ldots$ & $\ldots$ & $\ldots$ & $272.1,2.5$ \\
\hline 342 & 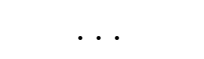 & $283.1,1.8$ & $286.6,2.1$ & & $\ldots$ & $\ldots$ & $\ldots$ & $\ldots$ & $284.8,2.7$ \\
\hline 343 & $282.9,2.0$ & & & $287.2,2.0$ & $\ldots$ & $\ldots$ & $\ldots$ & $\ldots$ & $285.0,3.1$ \\
\hline 344 & $\ldots$ & $\ldots$ & $\ldots$ & $\ldots$ & $\ldots$ & $268.8,1.9$ & $277.0,1.7$ & $\ldots$ & $273.1,5.0$ \\
\hline 346 & $\ldots$ & $52.1,1.9$ & $52.8,1.7$ & $52.2,1.9$ & $\ldots$ & $\ldots$ & $\ldots$ & $\ldots$ & $52.4,1.8$ \\
\hline 347 & & $285.4,1.9$ & $286.4,1.8$ & $287.3,1.9$ & $\ldots$ & $\ldots$ & $\ldots$ & $\ldots$ & $286.4,2.0$ \\
\hline 350 & $\ldots$ & $\ldots$ & $296.4,1.9$ & $\ldots$ & $\ldots$ & $296.5,2.0$ & $\ldots$ & $\ldots$ & $296.4,1.9$ \\
\hline 351 & $91.5,2.0$ & $91.9,2.1$ & $\ldots$ & $\ldots$ & $\ldots$ & $\ldots$ & $\ldots$ & $\ldots$ & $91.7,2.0$ \\
\hline 353 & $\ldots$ & $95.3,2.0$ & $\ldots$ & $97.0,2.0$ & $\ldots$ & $\ldots$ & $\ldots$ & $\ldots$ & $96.1,2.2$ \\
\hline 354 & & & $295.6,1.8$ & $\ldots$ & $296.5,2.0$ & $297.2,2.0$ & $294.0,1.7$ & $\ldots$ & $295.8,2.2$ \\
\hline 355 & & & $-52.2,1.9$ & & $\ldots$ & $-50.4,2.1$ & $\ldots$ & $\ldots$ & $-51.3,2.2$ \\
\hline 356 & $\ldots$ & $293.0,2.0$ & $297.6,1.9$ & $\ldots$ & $\ldots$ & $\ldots$ & $294.3,1.7$ & $\ldots$ & $294.8,2.8$ \\
\hline
\end{tabular}


Table 4-Continued

\begin{tabular}{|c|c|c|c|c|c|c|c|c|c|}
\hline ID & $\begin{array}{l}v_{h, 1}, \sigma_{1} \\
(\mathrm{~km} / \mathrm{s})\end{array}$ & $\begin{array}{c}v_{h, 2}, \sigma_{2} \\
(\mathrm{~km} / \mathrm{s})\end{array}$ & $\begin{array}{c}v_{h, 3}, \sigma_{3} \\
(\mathrm{~km} / \mathrm{s})\end{array}$ & $\begin{array}{l}v_{h, 4}, \sigma_{4} \\
(\mathrm{~km} / \mathrm{s})\end{array}$ & $\begin{array}{l}v_{h, 5}, \sigma_{5} \\
(\mathrm{~km} / \mathrm{s})\end{array}$ & $\begin{array}{c}v_{h, 6}, \sigma_{6} \\
(\mathrm{~km} / \mathrm{s})\end{array}$ & $\begin{array}{c}v_{h, 7}, \sigma_{7} \\
(\mathrm{~km} / \mathrm{s})\end{array}$ & $\begin{array}{c}v_{h, 8}, \sigma_{8} \\
(\mathrm{~km} / \mathrm{s})\end{array}$ & $\begin{array}{c}v_{a v g}, \sigma_{a v g} \\
(\mathrm{~km} / \mathrm{s})\end{array}$ \\
\hline 357 & & & $276.3,1.9$ & $272.0,2.1$ & & $278.3,2.0$ & $277.0,1.8$ & & $276.3,3.0$ \\
\hline 358 & $-18.6,1.9$ & $-22.3,1.9$ & $-24.1,1.9$ & & & & & & $-21.9,3.1$ \\
\hline 359 & $-20.9,1.9$ & $-19.1,2.0$ & $\ldots$ & $-21.8,1.8$ & $-23.6,2.0$ & $-22.6,1.8$ & $-22.6,1.7$ & & $-21.9,2.3$ \\
\hline 360 & $\ldots$ & $\ldots$ & $294.3,2.0$ & $297.4,2.0$ & $\ldots$ & $293.2,2.0$ & $\ldots$ & $\ldots$ & $294.8,2.8$ \\
\hline 361 & $260.0,1.9$ & $\ldots$ & $261.5,1.8$ & $\ldots$ & $\ldots$ & 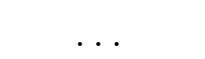 & $\ldots$ & $\ldots$ & $260.8,2.0$ \\
\hline 363 & $-13.2,2.0$ & $-13.4,1.8$ & $-14.1,1.8$ & $-13.0,1.9$ & & & & & $-13.4,1.9$ \\
\hline 366 & $274.8,2.0$ & $\ldots$ & $270.8,2.0$ & $\ldots$ & $\ldots$ & $\ldots$ & $\ldots$ & $\ldots$ & $272.8,3.0$ \\
\hline 367 & $\ldots$ & $\ldots$ & $\ldots$ & $55.6,2.0$ & $51.9,2.0$ & $\ldots$ & $\ldots$ & $\ldots$ & $53.8,2.8$ \\
\hline 368 & $\ldots$ & $280.1,1.9$ & $281.7,1.8$ & $280.4,2.0$ & $280.3,1.8$ & $282.4,1.9$ & $\ldots$ & $\ldots$ & $281.0,2.1$ \\
\hline 371 & $\ldots$ & $104.7,1.9$ & $103.2,1.8$ & $103.6,1.9$ & $\ldots$ & $\ldots$ & $\ldots$ & $\ldots$ & $103.8,2.0$ \\
\hline 373 & $\ldots$ & $41.2,1.9$ & $40.0,1.7$ & $38.7,1.9$ & $\ldots$ & $\ldots$ & & $\ldots$ & $40.0,2.1$ \\
\hline 374 & $\ldots$ & $\ldots$ & $292.4,1.9$ & $287.8,2.0$ & $\ldots$ & $\ldots$ & $\ldots$ & & $290.2,3.2$ \\
\hline 376 & $\ldots$ & $\ldots$ & $269.1,1.8$ & $263.5,2.1$ & $\ldots$ & $\ldots$ & $269.7,1.8$ & $\ldots$ & $268.2,3.4$ \\
\hline 377 & $\ldots$ & $\ldots$ & $283.4,1.9$ & $\ldots$ & $\ldots$ & $\ldots$ & $281.2,1.8$ & $\ldots$ & $282.3,2.2$ \\
\hline 378 & $\ldots$ & $\ldots$ & $297.1,1.8$ & $\ldots$ & $298.2,2.0$ & 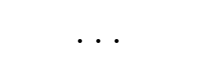 & $298.8,1.8$ & 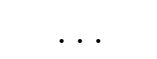 & $298.0,2.0$ \\
\hline 379 & $-3.5,2.0$ & $1.5,1.9$ & $5.4,1.9$ & $2.7,2.1$ & $2.3,1.9$ & & & & $2.1,3.5$ \\
\hline 384 & $\ldots$ & $\ldots$ & $28.6,1.9$ & $\ldots$ & $26.8,2.1$ & $25.1,2.1$ & $\ldots$ & $\ldots$ & $26.9,2.5$ \\
\hline 385 & $\ldots$ & $\ldots$ & $144.7,2.0$ & $\ldots$ & $135.7,2.0$ & $\ldots$ & $\ldots$ & $\ldots$ & $140.2,5.5$ \\
\hline 386 & $\ldots$ & $\ldots$ & $\ldots$ & $19.3,1.9$ & $18.3,2.1$ & $\ldots$ & $\ldots$ & $\ldots$ & $18.8,2.0$ \\
\hline 387 & $\ldots$ & $\ldots$ & $\ldots$ & $-6.2,1.9$ & $-2.8,1.9$ & $-7.1,1.7$ & & & $-5.6,2.7$ \\
\hline
\end{tabular}


aThe subscripts used in the headings of columns 2-7 refer to the configurations listed in Table 1: $1=\mathrm{Leo} \mathrm{I} / \mathrm{c} 1$; $2=$ Leo I/c2; $3=$ Leo I/c3; $4=$ Leo I/c4; $5=$ Leo I/c5; $6=$ Leo I/c6; $7=$ Leo I/c7; $8=$ Keck velocities from M98. 
Table 5. Summary of Observations of Leo I Targets ${ }^{\mathrm{a}}$

\begin{tabular}{|c|c|c|c|c|c|c|c|c|c|c|}
\hline ID $^{b}$ & $\begin{array}{c}R^{\mathrm{c}} \\
\left({ }^{\prime \prime}\right)\end{array}$ & $\begin{array}{r}P A^{\mathrm{c}} \\
(\mathrm{deg})\end{array}$ & $\begin{array}{c}v_{h} \\
(\mathrm{~km} / \mathrm{s})\end{array}$ & $\begin{array}{c}\sigma_{v_{h}} \\
(\mathrm{~km} / \mathrm{s})\end{array}$ & $\alpha_{J 2000}$ & $\delta_{J 2000}$ & I & $\mathrm{V}-\mathrm{I}$ & $\mathrm{SRC}^{\mathrm{d}}$ & $\begin{array}{c}\text { M98 } \\
\text { No. }^{\text {e }}\end{array}$ \\
\hline 1 & 2.3 & 77.2 & 283.3 & 1.6 & $10: 08: 27.15$ & $12: 18: 30.5$ & 18.34 & 1.27 & 7 & \\
\hline 2 & 6.6 & 358.7 & 294.9 & 1.9 & $10: 08: 26.99$ & $12: 18: 36.6$ & 18.34 & 1.28 & 6 & \\
\hline 3 & 11.6 & 329.6 & 274.1 & 1.8 & 10:08:26.60 & $12: 18: 40.0$ & 17.99 & 1.43 & 8 & 13 \\
\hline 4 & 28.4 & 48.0 & 281.4 & 1.8 & $10: 08: 28.44$ & $12: 18: 49.0$ & 17.89 & 1.40 & 8 & 12 \\
\hline 5 & 32.9 & 86.7 & 287.4 & 2.0 & 10:08:29.24 & 12:18:31.9 & 17.51 & 1.58 & 1234 & \\
\hline 6 & 35.4 & 158.2 & 264.8 & 1.9 & 10:08:27.90 & $12: 17: 57.1$ & 18.19 & 1.50 & 6 & \\
\hline 7 & 36.7 & 160.1 & 287.8 & 2.0 & 10:08:27.85 & $12: 17: 55.5$ & 17.89 & 1.63 & 7 & \\
\hline 8 & 43.3 & 143.7 & 288.4 & 2.0 & $10: 08: 28.75$ & $12: 17: 55.1$ & 18.55 & 1.26 & 5 & \\
\hline 9 & 46.9 & 299.3 & 280.8 & 1.8 & $10: 08: 24.21$ & $12: 18: 52.9$ & 18.67 & 1.35 & 7 & \\
\hline 10 & 51.1 & 15.5 & 291.6 & 1.7 & $10: 08: 27.93$ & $12: 19: 19.2$ & 18.31 & 1.35 & 7 & \\
\hline 11 & 52.2 & 226.4 & 292.9 & 1.8 & $10: 08: 24.42$ & $12: 17: 54.0$ & 18.30 & 1.39 & 8 & 19 \\
\hline 12 & 55.4 & 278.3 & 295.9 & 2.8 & $10: 08: 23.26$ & $12: 18: 38.0$ & 17.74 & 1.62 & 148 & 15 \\
\hline 13 & 58.5 & 170.6 & 293.9 & 2.0 & 10:08:27.65 & $12: 17: 32.3$ & 17.73 & 1.72 & 5 & \\
\hline 14 & 58.6 & 176.4 & 285.2 & 1.8 & $10: 08: 27.25$ & $12: 17:$ & 18.30 & 1.37 & 6 & \\
\hline 15 & 60.1 & 84.5 & 271.8 & 1.9 & 10:08:31.08 & $12: 18: 35.8$ & 18.52 & 1.37 & 6 & \\
\hline 16 & 60.2 & 279.4 & 283.6 & 2.1 & 10:08:22.95 & 12:18:39.8 & 18.32 & 1.34 & 58 & 14 \\
\hline 17 & 62.0 & 298.4 & 263.1 & 2.0 & 10:08:23.28 & $12: 18: 59.5$ & 18.29 & 1.43 & 6 & \\
\hline 18 & 63.1 & 247.2 & 278.9 & 2.1 & 10:08:23.03 & $12: 18: 05.6$ & 18.42 & 1.27 & 5 & \\
\hline 19 & 64.6 & 356.2 & 278.5 & 2.0 & 10:08:26.71 & $12: 19: 34.5$ & 18.28 & 1.46 & 6 & \\
\hline 20 & 65.5 & 244.8 & 297.6 & 1.9 & $10: 08: 22.96$ & $12: 18: 02.1$ & 18.53 & 1.24 & 6 & \\
\hline 21 & 67.4 & 327.4 & 278.6 & 2.0 & $10: 08: 24.52$ & $12: 19: 26.8$ & 18.72 & 1.32 & 6 & \\
\hline 22 & 67.8 & 44.1 & 285.4 & 1.8 & $10: 08: 30.22$ & $12: 19: 18.7$ & 18.19 & 1.43 & 8 & 10 \\
\hline 23 & 69.8 & 168.2 & 281.2 & 1.9 & $10: 08: 27.97$ & $12: 17: 21.7$ & 18.06 & 1.35 & 24 & \\
\hline 24 & 69.8 & 221.4 & 286.4 & 1.8 & 10:08:23.85 & $12: 17: 37.7$ & 18.50 & 1.34 & 6 & \\
\hline 25 & 71.3 & & 285.2 & 2. & $10: 08: 31.57$ & $12: 18: 54.4$ & 17.85 & 1.46 & 234 & \\
\hline 26 & 75.6 & 188.6 & 277.2 & 1.8 & $10: 08: 26.23$ & $12: 17: 15.2$ & 18.26 & 1.36 & 7 & \\
\hline 27 & 75.9 & 52.2 & 303.6 & 1.6 & 10:08:31.09 & $12: 19: 16.5$ & 18.22 & 1.50 & 7 & \\
\hline 28 & 77.9 & 302.2 & 283.9 & 2.0 & $10: 08: 22.50$ & $12: 19: 11.5$ & 18.21 & 1.41 & 348 & 11 \\
\hline 29 & 82.2 & 348.9 & 284.0 & 1.8 & 10:08:25.92 & $12: 19: 50.7$ & 18.61 & 1.24 & 7 & \\
\hline 30 & 85.6 & 106.3 & 277.9 & 1.8 & 10:08:32.61 & $12: 18: 06.0$ & 17.76 & 1.47 & 8 & 17 \\
\hline
\end{tabular}


Table 5-Continued

\begin{tabular}{|c|c|c|c|c|c|c|c|c|c|c|}
\hline $\mathrm{ID}^{\mathrm{b}}$ & $\begin{array}{c}R^{\mathrm{c}} \\
\left({ }^{\prime \prime}\right)\end{array}$ & $\begin{array}{r}P A^{\mathrm{c}} \\
(\mathrm{deg})\end{array}$ & $\begin{array}{c}v_{h} \\
(\mathrm{~km} / \mathrm{s})\end{array}$ & $\begin{array}{c}\sigma_{v_{h}} \\
(\mathrm{~km} / \mathrm{s})\end{array}$ & $\alpha_{J 2000}$ & $\delta_{J 2000}$ & I & V-I & $\mathrm{SRC}^{\mathrm{d}}$ & $\begin{array}{c}\text { M98 } \\
\text { No. }^{\mathrm{e}}\end{array}$ \\
\hline 31 & 86.8 & 109.9 & 289.3 & 2.4 & 10:08:32.57 & 12:18:00.5 & 18.03 & 1.38 & 138 & 18 \\
\hline 32 & 87.8 & 6.5 & 277.0 & 1.7 & 10:08:27.68 & $12: 19: 57.2$ & 18.42 & 1.32 & 7 & \\
\hline 33 & 87.9 & 212.6 & 279.5 & 1.7 & 10:08:23.77 & $12: 17: 15.9$ & 18.36 & 1.43 & 7 & \\
\hline 34 & 89.0 & 35.2 & 271.0 & 2.0 & 10:08:30.50 & $12: 19: 42.7$ & 18.91 & 1.32 & 5 & \\
\hline 35 & 91.1 & 228.6 & 275.7 & 1.7 & 10:08:22.34 & $12: 17: 29.7$ & 18.89 & 1.26 & 7 & \\
\hline 36 & 93.1 & 209.1 & 281.6 & 2.1 & 10:08:23.91 & $12: 17: 08.7$ & 17.67 & 1.57 & 1248 & 23 \\
\hline 37 & 93.5 & 102.4 & 292.7 & 2.0 & 10:08:33.23 & 12:18:09.9 & 18.32 & 1.35 & 5 & \\
\hline 38 & 99.1 & 312.8 & 275.2 & 2.2 & 10:08:22.04 & $12: 19: 37.4$ & 17.76 & 1.57 & 1258 & 9 \\
\hline 39 & 101.1 & 279.2 & 277.7 & 1.9 & 10:08:20.19 & $12: 18: 46.2$ & 18.24 & 1.40 & 6 & \\
\hline 40 & 101.1 & 344.4 & 300.3 & 6.5 & 10:08:25.14 & $12: 20: 07.4$ & 18.16 & 1.42 & 58 & 6 \\
\hline 41 & 103.6 & 175.5 & 277.9 & 1.8 & $10: 08: 27.55$ & $12: 16: 46.7$ & 18.33 & 1.38 & 8 & 25 \\
\hline 42 & 105.7 & 33.2 & 275.5 & 2.0 & 10:08:30.95 & 12:19:58.4 & 18.17 & 1.41 & 1 & \\
\hline 43 & 105.8 & 245.1 & 273.3 & 1.8 & 10:08:20.45 & $12: 17: 45.4$ & 18.26 & 1.52 & 8 & 20 \\
\hline 44 & 111.1 & 4.2 & 286.8 & 2.0 & $10: 08: 27.55$ & $12: 20: 20.8$ & 18.33 & 1.34 & 358 & 4 \\
\hline 45 & 113.0 & 173.9 & 287.8 & 1.8 & 10:08:27.82 & $12: 16: 37.6$ & 18.39 & 1.39 & 8 & 26 \\
\hline 46 & 113.0 & 310.4 & 289.6 & 1.8 & 10:08:21.13 & 12:19:43.3 & 18.30 & 1.43 & 8 & 7 \\
\hline 47 & 114.2 & 245.1 & 280.9 & 2.1 & 10:08:19.93 & $12: 17: 41.9$ & 17.24 & 1.84 & 124 & \\
\hline 48 & 115.4 & 298.1 & 299.3 & 2.3 & $10: 08: 20.05$ & $12: 19: 24.3$ & 18.35 & 1.37 & 25 & \\
\hline 49 & 115.6 & 128.2 & 291.5 & 2.9 & 10:08:33.20 & $12: 17: 18.5$ & 18.20 & 1.44 & 58 & 22 \\
\hline 50 & 115.9 & 191.5 & 288.0 & 2.0 & $10: 08: 25.43$ & $12: 16: 36.4$ & 18.74 & 1.30 & 6 & \\
\hline 51 & 117.4 & 289.7 & 284.7 & 1.8 & 10:08:19.46 & 12:19:09.6 & 19.12 & 1.17 & 7 & \\
\hline 52 & 120.0 & 10.6 & 289.1 & 1.8 & $10: 08: 28.5$ & $12: 20: 28$ & 18.09 & 1.53 & 8 & 3 \\
\hline 53 & 120.3 & 31.9 & 282.7 & 1.9 & 10:08:31.34 & $12: 20: 12.1$ & 18.63 & 1.11 & 6 & \\
\hline 54 & 122.1 & 103.5 & 289.2 & 2.0 & 10:08:35.10 & $12: 18: 01.4$ & 19.20 & 1.21 & 6 & \\
\hline 55 & 123.8 & 326.8 & 281.1 & 2.0 & 10:08:22.37 & $12: 20: 13.5$ & 19.06 & 1.29 & 6 & \\
\hline 56 & 124.9 & 22.8 & 293.3 & 1.8 & 10:08:30.30 & $12: 20: 25.2$ & 18.82 & 1.20 & 7 & \\
\hline 57 & 127.0 & 168.6 & 291.8 & 2.1 & 10:08:28.71 & $12: 16: 25.5$ & 17.73 & 1.53 & 12348 & 27 \\
\hline 58 & 127.5 & 279.3 & 269.1 & 1.7 & 10:08:18.41 & $12: 18: 50.5$ & 18.19 & 1.46 & 3 & \\
\hline 59 & 130.5 & 247.0 & 284.0 & 1.9 & 10:08:18.80 & $12: 17: 39.0$ & 19.22 & 1.25 & 6 & \\
\hline 60 & 130.6 & 183.3 & 296.1 & 1.8 & 10:08:26.49 & 12:16:19.6 & 18.25 & 1.24 & 8 & 28 \\
\hline
\end{tabular}


Table 5-Continued

\begin{tabular}{|c|c|c|c|c|c|c|c|c|c|c|}
\hline ID $^{b}$ & $\begin{array}{l}R^{\mathrm{c}} \\
\left({ }^{\prime \prime}\right)\end{array}$ & $\begin{array}{r}P A^{\mathrm{c}} \\
(\mathrm{deg})\end{array}$ & $\begin{array}{c}v_{h} \\
(\mathrm{~km} / \mathrm{s})\end{array}$ & $\begin{array}{c}\sigma_{v_{h}} \\
(\mathrm{~km} / \mathrm{s})\end{array}$ & $\alpha_{J 2000}$ & $\delta_{J 2000}$ & I & $\mathrm{V}-\mathrm{I}$ & $\mathrm{SRC}^{\mathrm{d}}$ & $\begin{array}{l}\text { M98 } \\
\text { No. }{ }^{\mathrm{e}}\end{array}$ \\
\hline 61 & 131.8 & 88.9 & 267.6 & 2.1 & 10:08:35.99 & $12: 18: 32.5$ & 18.36 & 1.37 & 4 & \\
\hline 62 & 132.4 & 326.9 & & 2.6 & 10:08:22.06 & & 17.88 & 1.41 & 2348 & 5 \\
\hline 63 & 133.2 & 157.0 & 301.4 & 1.9 & 10:08:30.55 & $12: 16: 27.4$ & 18.64 & 1.21 & 7 & \\
\hline 64 & 134.4 & 19.8 & 289.0 & 2.0 & 10:08:30.11 & $12: 20: 3$ & 18.59 & 1.37 & 6 & \\
\hline 65 & 135.0 & 344.5 & 297.6 & 2.7 & 10:08:24.53 & $12: 20: 40.1$ & 17.56 & 1.62 & 1234 & \\
\hline 66 & 135.2 & 59.3 & 286.8 & 2.0 & 10:08:34.93 & $12: 19: 39.0$ & 17.83 & 1.54 & 48 & 0 \\
\hline 67 & 137.6 & 232.1 & 288.5 & 1.7 & 59 & $12: 17: 0$ & 18.45 & 1.39 & 7 & \\
\hline 68 & 138.0 & 118.3 & 311.1 & 3.7 & 10:08: & $12: 17:$ & 17.67 & 1.72 & 158 & 21 \\
\hline 69 & 138.0 & 233.2 & 284.2 & & 10:08 & $12: 1$ & 17.72 & 1.72 & 8 & 24 \\
\hline 70 & 141.6 & 167.0 & 282.0 & 1.9 & 10:08 & $12: 1$ & 18.63 & 1.29 & 6 & \\
\hline 71 & 142.8 & 224.1 & 284.4 & 1.9 & $10: 0 \varepsilon$ & $12: 1$ & 18.34 & 1.42 & 5 & \\
\hline 72 & 142.8 & 228.4 & & 2.0 & & $12: 1$ & 18.54 & 1.36 & 4 & \\
\hline 73 & 143.3 & 249.7 & 296.5 & 1.7 & 10:08:17.83 & $12: 17: 40.3$ & 18.45 & 1.37 & 7 & \\
\hline 74 & 144.7 & 304.0 & 275.6 & 1.8 & 10:08:18.81 & $12: 19: 50.8$ & 19.01 & 1.28 & 7 & \\
\hline 75 & 145.0 & 275.9 & 283.4 & 1.8 & 10:08: & $12: 18: 44.9$ & 18.20 & 1.39 & 5 & \\
\hline 76 & 145.4 & 188.6 & 273.7 & 1.8 & $10: 0$ & $12: 1$ & 19.38 & 1.17 & 7 & \\
\hline 77 & 146.2 & 278.7 & 281.7 & 2.9 & & 12:18: & 17.77 & 1.49 & 14 & \\
\hline 78 & 146.3 & 264.1 & 286.9 & 1.8 & 10:08:17.07 & $12: 18: 14.9$ & 17.94 & 1.55 & 8 & 16 \\
\hline 79 & 146.4 & 267.9 & 277.6 & 1.6 & 10:08:17.02 & $12: 18: 24.7$ & 18.44 & 1.34 & 7 & \\
\hline 80 & 147.4 & 302.8 & 287.2 & 1.9 & $10: 08: 18.55$ & $12: 19: 49.9$ & 18.34 & 1.39 & 6 & \\
\hline 81 & 148.6 & 353.8 & 269.8 & 1.9 & 10:08:25.91 & $12: 20: 57.7$ & 18.99 & 1.21 & 6 & \\
\hline 82 & 150.3 & 334.4 & 285.2 & 1.7 & 10:08:22.56 & $12: 20: 45.5$ & 18.74 & 1.28 & 7 & \\
\hline 83 & 151.8 & 108.3 & 281.6 & 1.9 & 10:08:36.83 & $12: 17: 42.3$ & 18.32 & 1.35 & 2 & \\
\hline 84 & 151.9 & 213.1 & 278.9 & 2.0 & 10:08:21 & $12: 16: 22.8$ & 18.47 & 1.29 & 5 & \\
\hline 85 & 152.6 & 163.1 & 292.5 & 2.8 & 10:08:30.02 & $12: 16: 04.0$ & 18.24 & 1.46 & 18 & 31 \\
\hline 86 & 155.0 & 208.7 & 293.9 & 1.8 & 10:08:21.92 & $12: 16: 14.0$ & 18.23 & 1.44 & 8 & 30 \\
\hline 87 & 157.4 & 172.5 & 276.4 & 1.8 & 10:08:28.41 & $12: 15: 54.0$ & 18.23 & 1.36 & 8 & 32 \\
\hline 88 & 157.4 & 221.0 & & 2.1 & & $12: 16: 31.2$ & 19.66 & 1.16 & 6 & \\
\hline 89 & 158.4 & & & 1.9 & & $12: 19: 24.4$ & 17.82 & 1.46 & 1234 & \\
\hline 90 & 161.0 & 220.1 & 273.9 & 1.6 & 10:08:19.92 & $12: 16: 26.9$ & 18.45 & 1.37 & 7 & \\
\hline
\end{tabular}


Table 5-Continued

\begin{tabular}{|c|c|c|c|c|c|c|c|c|c|c|}
\hline ID $^{b}$ & $\begin{array}{l}R^{\mathrm{c}} \\
\left({ }^{\prime \prime}\right)\end{array}$ & $\begin{array}{r}P A^{\mathrm{c}} \\
(\mathrm{deg})\end{array}$ & $\begin{array}{c}v_{h} \\
(\mathrm{~km} / \mathrm{s})\end{array}$ & $\begin{array}{c}\sigma_{v_{h}} \\
(\mathrm{~km} / \mathrm{s})\end{array}$ & $\alpha_{J 2000}$ & $\delta_{J 2000}$ & I & $\mathrm{V}-\mathrm{I}$ & $\mathrm{SRC}^{\mathrm{d}}$ & $\begin{array}{l}\text { M98 } \\
\text { No. }{ }^{\text {e }}\end{array}$ \\
\hline 91 & 165.4 & 35.3 & 295.6 & 2.5 & 10:08:33.52 & $12: 20: 45.0$ & 18.11 & 1.37 & 358 & 2 \\
\hline 92 & 167.6 & 3.7 & 293.3 & 2.0 & 10:08:27.74 & $12: 21: 17.2$ & 18.21 & 1.36 & 134 & \\
\hline 93 & 168.7 & 235.1 & 281.9 & 1.8 & $10: 08: 17.56$ & $12: 16: 53.4$ & 18.10 & 1.34 & 5 & \\
\hline 94 & 169.7 & 5.0 & 291.9 & 1.9 & 10:08:28.00 & $12: 21: 19.1$ & 18.71 & 1.40 & 6 & \\
\hline 95 & 173.1 & 98.2 & 289.1 & 2.1 & 10:08:38.69 & 12:18:05.2 & 18.29 & 1.39 & 13 & \\
\hline 96 & 177.1 & 55.0 & 295.0 & 1.8 & 10:08:36.90 & 12:20:11.5 & 17.68 & 1.57 & 3 & \\
\hline 97 & 178.4 & 253.3 & 271.2 & 1.9 & 10:08:15.34 & $12: 17: 38.6$ & 18.22 & 1.54 & 5 & \\
\hline 98 & 179.8 & 98.3 & 278.0 & 1.8 & 10:08:39.14 & $12: 18: 04.1$ & 19.10 & 1.12 & 7 & \\
\hline 99 & 180.2 & 320.3 & 270.7 & 1.7 & 10:08:19.15 & $12: 20: 48.7$ & 18.56 & 1.36 & 7 & \\
\hline 100 & 180.7 & 260.9 & 289.5 & 2.0 & $10: 08: 14.83$ & $12: 18: 01.3$ & 18.41 & 1.36 & 5 & \\
\hline 101 & 180.8 & 302.5 & 282.4 & 1.6 & 10:08:16.59 & 12:20:07.1 & 17.75 & 1.55 & 3 & \\
\hline 102 & 181.3 & 328.1 & 271.0 & 4.9 & 0.46 & $12: 21$ & 18.00 & 1.49 & 28 & \\
\hline 103 & 183.2 & 19.7 & 291.6 & 2.1 & 10:08:31.22 & $12: 21: 22.4$ & 19.43 & 1.24 & 1 & \\
\hline 104 & 184.8 & 261.4 & 292.6 & 2.1 & 10:08:14.53 & 12:18:02.4 & 17.51 & 1.72 & 2 & \\
\hline 105 & 185.0 & 74.9 & 273.5 & 1.9 & 10:08:39.19 & $12: 19: 18.1$ & 18.34 & 1.34 & 5 & \\
\hline 106 & 187.9 & 349.2 & 285.4 & 2.0 & 10:08:24.60 & $12: 21: 34.6$ & 19.14 & 1.21 & 6 & \\
\hline 107 & 189.5 & 144.5 & 300.8 & 2.1 & 10:08:34.50 & $12: 15: 5$ & 17.77 & 1.58 & 124 & \\
\hline 108 & 192.1 & 84.9 & 284.9 & 1.9 & $10: 08: 40.06$ & $12: 18: 47.0$ & 18.28 & 1.40 & 5 & \\
\hline 109 & 192.9 & 37.4 & 296.2 & 3.9 & 10:08:34.99 & $12: 21: 03.3$ & 17.38 & 1.77 & 123 & \\
\hline 110 & 192.9 & 225.9 & 279.6 & 1.8 & 10:08:17.55 & $12: 16: 15.7$ & 18.15 & 1.55 & 8 & 29 \\
\hline 111 & 194.1 & 197.4 & 275.8 & 1.9 & $10: 08: 23.03$ & $12: 15: 24.8$ & 18.54 & 1.48 & 6 & \\
\hline 112 & 194.9 & 48.6 & 272.4 & 2.1 & 10:08:36.97 & $12: 20: 39.0$ & 18.65 & 1.11 & 5 & \\
\hline 113 & 195.3 & 150.0 & 286.9 & 2.0 & 10:08:33.66 & $12: 15: 40.9$ & 17.72 & 1.63 & 358 & 33 \\
\hline 114 & 195.6 & 236.3 & 282.4 & 1.7 & 10:08 & $12: 16: 41.4$ & 19.17 & 1.19 & 7 & \\
\hline 115 & 195.9 & 254.1 & 302.8 & 1.9 & 10:08:14.14 & $12: 17: 36.4$ & 18.88 & 1.18 & 6 & \\
\hline 116 & 198.0 & 110.8 & 273.3 & 2.0 & 10:08:39.63 & $12: 17: 19.8$ & 18.33 & 1.36 & 134 & \\
\hline 117 & 198.5 & 318.6 & 277.7 & 2.0 & 10:08:18.05 & $12: 20: 59.0$ & 18.68 & 1.36 & 1 & \\
\hline 118 & 200.3 & 62.3 & 282.6 & 1.9 & 10:08:39.10 & $12: 20: 03.1$ & 18.17 & 1.39 & 1 & \\
\hline 119 & 201.7 & 359.8 & 275.6 & 2.4 & $10: 08: 26.94$ & $12: 21: 51.7$ & 18.85 & 1.27 & 23 & \\
\hline 120 & 201.8 & 137.1 & 272.1 & 1.9 & 10:08:36.37 & $12: 16: 02.1$ & 18.64 & 1.28 & 6 & \\
\hline
\end{tabular}


Table 5-Continued

\begin{tabular}{|c|c|c|c|c|c|c|c|c|c|c|}
\hline ID ${ }^{b}$ & $\begin{array}{l}R^{\mathrm{c}} \\
\left({ }^{\prime \prime}\right)\end{array}$ & $\begin{array}{r}P A^{\mathrm{c}} \\
(\mathrm{deg})\end{array}$ & $\begin{array}{c}v_{h} \\
(\mathrm{~km} / \mathrm{s})\end{array}$ & $\begin{array}{c}\sigma_{v_{h}} \\
(\mathrm{~km} / \mathrm{s})\end{array}$ & $\alpha_{J 2000}$ & $\delta_{J 2000}$ & I & $\mathrm{V}-\mathrm{I}$ & $\mathrm{SRC}^{\mathrm{d}}$ & $\begin{array}{l}\text { M98 } \\
\text { No. }\end{array}$ \\
\hline 121 & 202.0 & 339.7 & 280.9 & 2.0 & 10:08:22.21 & $12: 21: 39.4$ & 18.71 & 1.31 & 2 & \\
\hline 122 & 202.2 & 276.3 & 285.5 & 2.5 & 10:08:13.29 & $12: 18: 52.3$ & 18.35 & 1.40 & 23 & \\
\hline 123 & 203.8 & 301.5 & 269.4 & 1.9 & 10:08:15.15 & $12: 20: 16.6$ & 19.31 & 1.27 & 7 & \\
\hline 124 & 204.2 & 306.9 & 277.5 & 3.6 & 10:08:15.85 & $12: 20: 32.5$ & 17.59 & 1.57 & 134 & \\
\hline 125 & 204.3 & 32.1 & 270.5 & 2.0 & 10:08:34.42 & $12: 21: 23.0$ & 19.02 & 1.30 & 4 & \\
\hline 126 & 204.7 & 317.6 & 268.9 & 2.0 & $10: 08: 17.58$ & $12: 21: 01.2$ & 18.78 & 1.36 & 6 & \\
\hline 127 & 204.8 & 274.7 & 287.9 & 1.8 & 10:08:13.07 & $12: 18: 46.9$ & 19.14 & 1.25 & 7 & \\
\hline 128 & 205.5 & 96.6 & 288.6 & 3.2 & 10:08:40.93 & 12:18:06.4 & 18.23 & 1.43 & 124 & \\
\hline 129 & 208.7 & 16.2 & 288.5 & 1.9 & 10:08:30.98 & $12: 21: 50.4$ & 19.03 & 1.28 & 5 & \\
\hline 130 & 209.6 & 18.2 & 267.5 & 2.1 & 10:08:31.47 & $12: 21: 49.1$ & 19.20 & 1.27 & 2 & \\
\hline 131 & 211.0 & 136.3 & 293.5 & 1.8 & 10:08:36.95 & $12: 15: 57.5$ & 18.58 & 1.29 & 3 & \\
\hline 132 & 214.8 & 178.7 & 273.0 & 1.8 & 10:08:27.34 & $12: 14: 55.3$ & 19.01 & 1.23 & 7 & \\
\hline 133 & 215.3 & 335.3 & 287.1 & 2.1 & 10:08:20.86 & $12: 21: 45.6$ & 18.78 & 1.30 & 5 & \\
\hline 134 & 215.4 & 43.6 & 292.2 & 1.8 & 10:08:37.14 & $12: 21: 05.9$ & 19.03 & 1.26 & 7 & \\
\hline 135 & 215.8 & 225.8 & 277.9 & 2.0 & 10:08:16.45 & $12: 15: 59.4$ & 18.28 & 1.40 & 245 & \\
\hline 136 & 217.3 & 344.8 & 291.8 & 1.8 & 10:08:23.12 & $12: 21: 59.7$ & 18.85 & 1.29 & 7 & \\
\hline 137 & 217.7 & 261.6 & 273.6 & 1.7 & 10:08:12.31 & $12: 17: 58.0$ & 18.64 & 1.30 & 7 & \\
\hline 138 & 218.4 & 49.8 & 289.6 & 1.7 & $10: 08: 38.38$ & $12: 20: 51.0$ & 18.70 & 1.35 & 7 & \\
\hline 139 & 218.6 & 187.7 & -5.4 & 2.6 & $10: 08: 25.00$ & $12: 14: 53.4$ & 18.57 & 1.22 & 125 & \\
\hline 140 & 220.5 & 152.5 & 287.5 & 1.9 & 10:08:33.94 & $12: 15: 14.4$ & 18.19 & 1.41 & 4 & \\
\hline 141 & 220.6 & 327.5 & 281.3 & 1.9 & 10:08:18.92 & $12: 21: 36.1$ & 18.95 & 1.29 & 2 & \\
\hline 142 & 221.7 & 145.4 & -10.0 & 2.0 & $10: 08: 35.59$ & $12: 15: 27.5$ & 18.65 & 1.34 & 1 & \\
\hline 143 & 221.8 & 307.1 & 279.4 & 1.9 & 10:08:14.93 & $12: 20: 43.8$ & 18.18 & 1.46 & 5 & \\
\hline 144 & 225.5 & 297.4 & 286.3 & 2.0 & 10:08:13.33 & $12: 20: 13.6$ & 18.38 & 1.38 & 2 & \\
\hline 145 & 226.0 & 134.3 & 282.3 & 1.9 & $10: 08: 38.04$ & $12: 15: 52.2$ & 18.05 & 1.45 & 5 & \\
\hline 146 & 226.1 & 279.5 & -14.3 & 2.1 & 10:08:11.78 & 12:19:07.1 & 19.12 & 1.15 & 1 & \\
\hline 147 & 226.3 & 163.4 & 279.3 & 2.0 & $10: 08: 31.40$ & $12: 14: 53.1$ & 19.03 & 1.18 & 1 & \\
\hline 148 & 226.6 & 119.7 & -14.7 & 2.0 & 10:08:40.43 & $12: 16: 37.8$ & 18.44 & 1.37 & 1 & \\
\hline 149 & 227.1 & 184.5 & 289.5 & 1.8 & 10:08:25.79 & $12: 14: 43.6$ & 18.52 & 1.37 & 3 & \\
\hline 150 & 227.6 & 244.0 & -7.4 & 2.1 & 10:08:13.04 & $12: 16: 50.3$ & 17.55 & 1.60 & 1234 & \\
\hline
\end{tabular}


Table 5-Continued

\begin{tabular}{|c|c|c|c|c|c|c|c|c|c|c|}
\hline ID $^{b}$ & $\begin{array}{l}R^{\mathrm{c}} \\
\left({ }^{\prime \prime}\right)\end{array}$ & $\begin{array}{r}P A^{\mathrm{c}} \\
(\mathrm{deg})\end{array}$ & $\begin{array}{c}v_{h} \\
(\mathrm{~km} / \mathrm{s})\end{array}$ & $\begin{array}{c}\sigma_{v_{h}} \\
(\mathrm{~km} / \mathrm{s})\end{array}$ & $\alpha_{J 2000}$ & $\delta_{J 2000}$ & I & $\mathrm{V}-\mathrm{I}$ & $\mathrm{SRC}^{\mathrm{d}}$ & $\begin{array}{l}\text { M98 } \\
\text { No. }\end{array}$ \\
\hline 151 & 228.8 & 54.5 & -379.4 & 2.1 & 10:08:39.71 & $12: 20: 42.8$ & 19.33 & 1.21 & 2 & \\
\hline 152 & 229.4 & 343.6 & -19.5 & 2.0 & 10:08:22.58 & $12: 22: 10.1$ & 19.36 & 1.19 & 1 & \\
\hline 153 & 230.7 & 74.2 & 270.7 & 3.7 & $10: 08: 42.15$ & $12: 19: 32.7$ & 17.87 & 1.47 & 124 & \\
\hline 154 & 230.8 & 231.9 & -21.0 & 2.0 & 10:08:14.60 & $12: 16: 07.7$ & 18.21 & 1.31 & 1 & \\
\hline 155 & 233.0 & 356.6 & 28.0 & 1.9 & 10:08:26.05 & $12: 22: 22.6$ & 19.38 & 1.00 & 3 & \\
\hline 156 & 233.9 & 189.2 & 268.3 & 2.0 & 10:08:24.46 & $12: 14: 39.1$ & 18.60 & 1.37 & 6 & \\
\hline 157 & 234.7 & 134.8 & 273.4 & 4.3 & 10:08:38.37 & $12: 15: 44.7$ & 17.83 & 1.57 & 14 & \\
\hline 158 & 239.0 & 32.2 & 285.2 & 1.7 & 10:08:35.70 & $12: 21: 52.2$ & 18.88 & 1.20 & 7 & \\
\hline 159 & 240.0 & 6.9 & 279.5 & 1.9 & $10: 08: 28.97$ & $12: 22: 28.3$ & 19.46 & 1.24 & 7 & \\
\hline 160 & 241.1 & 316.3 & 295.0 & 1.8 & 10:08:15.63 & $12: 21: 24.3$ & 19.45 & 1.27 & 7 & \\
\hline 161 & 241.7 & 200.0 & 282.3 & 3.7 & $10: 08: 21.37$ & $12: 14: 42.8$ & 17.43 & 1.70 & 1234 & \\
\hline 162 & 243.0 & 140.7 & 270.8 & 2.1 & $10: 08: 37.50$ & $12: 15: 21.9$ & 19.24 & 1.25 & 6 & \\
\hline 163 & 244.3 & 205.1 & 287.9 & 1.9 & 10:08:19.92 & $12: 14: 48.8$ & 18.24 & 1.38 & 5 & \\
\hline 164 & 244.4 & 134.9 & 272.1 & 1.7 & 10:08:38.81 & $12: 15: 37.5$ & 18.69 & 1.21 & 7 & \\
\hline 165 & 244.9 & 17.2 & 288.2 & 2.0 & 10:08:31.95 & 12:22:23.9 & 19.60 & 1.14 & 7 & \\
\hline 166 & 247.4 & 194.6 & 281.9 & 2.0 & 10:08:22.74 & $12: 14: 30.6$ & 18.31 & 1.39 & 5 & \\
\hline 167 & 247.4 & 254.6 & 278.8 & 1.8 & 10:08:10.72 & $12: 17: 24.4$ & 17.86 & 1.49 & 3 & \\
\hline 168 & 251.2 & 16.4 & 294.9 & 2.1 & 10:08:31.85 & $12: 22: 30.9$ & 18.89 & 1.30 & 4 & \\
\hline 169 & 253.2 & 154.7 & 280.7 & 1.8 & $10: 08: 34.37$ & $12: 14: 41.0$ & 19.25 & 1.24 & 7 & \\
\hline 170 & 254.5 & 338.0 & 283.6 & 3.3 & 10:08:20.49 & $12: 22: 25.9$ & 18.87 & 1.27 & 23 & \\
\hline 171 & 258.4 & 89.4 & 298.8 & 3.2 & $10: 08: 44.63$ & 12:18:32.8 & 18.18 & 1.43 & 24 & \\
\hline 172 & 258.7 & 345.2 & 276.1 & 1.8 & $10: 08: 22.48$ & $12: 22: 40.1$ & 18.86 & 1.28 & 3 & \\
\hline 173 & 259.3 & 162.7 & 287.6 & 2.1 & $10: 08: 32.25$ & $12: 14: 22.4$ & 19.35 & 1.21 & 3 & \\
\hline 174 & 260.3 & 70.3 & 298.9 & 1.9 & 10:08:43.72 & $12: 19: 57.8$ & 18.31 & 1.38 & 5 & \\
\hline 175 & 260.3 & 141.9 & 286.2 & 2.1 & $10: 08: 37.96$ & $12: 15: 05.2$ & 18.64 & 1.33 & 1 & \\
\hline 176 & 263.1 & 159.7 & 289.0 & 1.7 & 10:08:33.24 & $12: 14: 23.3$ & 18.48 & 1.37 & 7 & \\
\hline 177 & 263.5 & 179.7 & 290.3 & 2.0 & 10:08:27.09 & 12:14:06.5 & 18.48 & 1.40 & 1 & \\
\hline 178 & 264.2 & 181.1 & 278.2 & 2.1 & 10:08:26.65 & $12: 14: 05.8$ & 18.80 & 1.33 & 5 & \\
\hline 179 & 266.3 & 104.6 & 289.7 & 2.3 & $10: 08: 44.58$ & $12: 17: 22.8$ & 17.86 & 1.50 & 1234 & \\
\hline 180 & 269.1 & 96.9 & 272.3 & 2.1 & $10: 08: 45.23$ & $12: 17: 57.6$ & 18.74 & 1.34 & 5 & \\
\hline
\end{tabular}


Table 5-Continued

\begin{tabular}{|c|c|c|c|c|c|c|c|c|c|c|}
\hline ID $^{b}$ & $\begin{array}{l}R^{\mathrm{c}} \\
\left({ }^{\prime \prime}\right)\end{array}$ & $\begin{array}{r}P A^{\mathrm{c}} \\
(\mathrm{deg})\end{array}$ & $\begin{array}{c}v_{h} \\
(\mathrm{~km} / \mathrm{s})\end{array}$ & $\begin{array}{c}\sigma_{v_{h}} \\
(\mathrm{~km} / \mathrm{s})\end{array}$ & $\alpha_{J 2000}$ & $\delta_{J 2000}$ & I & $\mathrm{V}-\mathrm{I}$ & $\mathrm{SRC}^{\mathrm{d}}$ & $\begin{array}{l}\text { M98 } \\
\text { No. }\end{array}$ \\
\hline 181 & 269.3 & 201.4 & 277.9 & 1.7 & 10:08:20.30 & $12: 14: 19.2$ & 18.75 & 1.35 & 7 & \\
\hline 182 & 271.0 & 72.3 & -19.3 & 2.0 & 10:08:44.62 & $12: 19: 52.2$ & 18.93 & 1.28 & 1 & \\
\hline 183 & 274.3 & 221.0 & 296.7 & 1.9 & 10:08:14.72 & $12: 15: 03.0$ & 18.56 & 1.38 & 4 & \\
\hline 184 & 278.5 & 168.8 & 288.6 & 2.1 & 10:08:30.68 & $12: 13: 56.8$ & 18.96 & 1.21 & 5 & \\
\hline 185 & 278.7 & 301.5 & 286.3 & 2.1 & 10:08:10.79 & $12: 20: 55.7$ & 18.21 & 1.50 & 1237 & \\
\hline 186 & 282.0 & 66.8 & 288.4 & 1.9 & 10:08:44.69 & $12: 20: 20.9$ & 18.21 & 1.39 & 4 & \\
\hline 187 & 282.5 & 148.2 & 282.9 & 2.1 & 10:08:37.16 & $12: 14: 29.9$ & 18.39 & 1.36 & 1 & \\
\hline 188 & 283.7 & 281.0 & 281.7 & 2.1 & 10:08:08.00 & $12: 19: 24.3$ & 18.86 & 1.33 & 5 & \\
\hline 189 & 284.8 & 161.4 & 277.7 & 1.9 & 10:08:33.19 & $12: 13: 60.0$ & 18.62 & 1.36 & 6 & \\
\hline 190 & 286.8 & 159.0 & 0.5 & 2.1 & 10:08:34.00 & 12:14:02.2 & 18.23 & 1.45 & 1 & \\
\hline 191 & 288.4 & 171.6 & 298.3 & 1.8 & $10: 08: 29.86$ & $12: 13: 44.7$ & 19.17 & 1.28 & 7 & \\
\hline 192 & 289.6 & 226.6 & 299.4 & 2.1 & $10: 08: 12.64$ & $12: 15: 11.1$ & 19.49 & 1.23 & 57 & \\
\hline 193 & 293.1 & 196.3 & 270.5 & 1.9 & 10:08:21.40 & $12: 13: 48.6$ & 17.69 & 1.68 & 1 & \\
\hline 194 & 293.8 & 153.3 & 198.8 & 2.0 & 10:08:36.00 & 12:14:07.5 & 19.01 & 1.18 & 5 & \\
\hline 195 & 294.5 & 178.3 & 276.8 & 4.1 & $10: 08: 27.58$ & 12:13:35.6 & 18.80 & 1.35 & 134 & \\
\hline 196 & 295.3 & 54.0 & 275.8 & 2.0 & 10:08:43.30 & $12: 21: 23.6$ & 18.52 & 1.37 & 5 & \\
\hline 197 & 295.7 & 211.8 & 283.3 & 1.6 & 10:08:16.37 & $12: 14: 18.7$ & 18.42 & 1.37 & 7 & \\
\hline 198 & 297.1 & 88.6 & 283.9 & 2.0 & $10: 08: 47.27$ & $12: 18: 37.1$ & 18.60 & 1.31 & 1 & \\
\hline 199 & 298.6 & 292.1 & -16.6 & 2.0 & 10:08:08.12 & $12: 20: 22.3$ & 19.34 & 1.24 & 1 & \\
\hline 200 & 299.0 & 187.1 & 275.0 & 1.7 & 10:08:24.49 & 12:13:33.3 & 18.92 & 1.34 & 7 & \\
\hline 201 & 299.1 & 132.2 & 276.3 & 2.1 & 10:08:42.11 & 12:15:08.9 & 19.18 & 1.25 & 1 & \\
\hline 202 & 299.3 & 213.3 & 97.2 & 3.0 & 10:08:15.80 & $12: 14: 19.7$ & 17.75 & 1.55 & 123 & \\
\hline 203 & 300.0 & 234.0 & 290.1 & 1.9 & 10:08:10.43 & $12: 15: 33.8$ & 18.59 & 1.37 & 6 & \\
\hline 204 & 300.3 & 28.0 & 294.5 & 2.0 & 10:08:36.63 & $12: 22: 55.1$ & 18.55 & 1.39 & 5 & \\
\hline 205 & 301.0 & 262.2 & -5.1 & 2.1 & 10:08:06.65 & $12: 17: 49.1$ & 18.30 & 1.48 & 1 & \\
\hline 206 & 301.5 & 39.8 & 271.9 & 2.1 & 10:08:40.17 & $12: 22: 21.6$ & 18.50 & 1.32 & 1 & \\
\hline 207 & 301.8 & 336.4 & 283.3 & 2.1 & $10: 08: 18.75$ & $12: 23: 06.5$ & 19.20 & 1.23 & 2 & \\
\hline 208 & 301.9 & 119.9 & 276.5 & 1.9 & 10:08:44.86 & $12: 15: 59.5$ & 18.59 & 1.38 & 6 & \\
\hline 209 & 302.2 & 112.7 & 287.5 & 2.1 & 10:08:46.02 & $12: 16: 33.4$ & 19.17 & 1.26 & 6 & \\
\hline 210 & 304.2 & 331.8 & 280.3 & 2.0 & $10: 08: 17.20$ & $12: 22: 58.2$ & 19.60 & 1.20 & 7 & \\
\hline
\end{tabular}


Table 5-Continued

\begin{tabular}{|c|c|c|c|c|c|c|c|c|c|c|}
\hline ID $^{b}$ & $\begin{array}{l}R^{\mathrm{c}} \\
\left({ }^{\prime \prime}\right)\end{array}$ & $\begin{array}{r}P A^{\mathrm{c}} \\
(\mathrm{deg})\end{array}$ & $\begin{array}{c}v_{h} \\
(\mathrm{~km} / \mathrm{s})\end{array}$ & $\begin{array}{c}\sigma_{v_{h}} \\
(\mathrm{~km} / \mathrm{s})\end{array}$ & $\alpha_{J 2000}$ & $\delta_{J 2000}$ & I & $\mathrm{V}-\mathrm{I}$ & $\mathrm{SRC}^{\mathrm{d}}$ & $\begin{array}{l}\text { M98 } \\
\text { No. }\end{array}$ \\
\hline 211 & 304.6 & 19.8 & 292.4 & 2.1 & 10:08:34.04 & $12: 23: 16.6$ & 18.62 & 1.38 & 3 & \\
\hline 212 & 304.6 & 116.9 & 287.5 & 1.9 & 10:08:45.54 & $12: 16: 12.3$ & 18.57 & 1.40 & 5 & \\
\hline 213 & 305.1 & 304.8 & 281.6 & 4.5 & 10:08:09.91 & $12: 21: 24.2$ & 18.70 & 1.34 & 23 & \\
\hline 214 & 305.7 & 90.1 & 261.5 & 2.6 & 10:08:47.86 & $12: 18: 29.6$ & 18.48 & 1.36 & 34 & \\
\hline 215 & 306.7 & 110.3 & 279.2 & 1.9 & 10:08:46.63 & $12: 16: 43.7$ & 19.25 & 1.22 & 7 & \\
\hline 216 & 307.6 & 118.7 & 279.1 & 2.0 & 10:08:45.40 & $12: 16: 02.1$ & 18.46 & 1.40 & 14 & \\
\hline 217 & 307.6 & 261.7 & 276.3 & 1.9 & 10:08:06.23 & $12: 17: 45.4$ & 19.08 & 1.22 & 3 & \\
\hline 218 & 308.6 & 289.6 & 285.9 & 2.0 & 10:08:07.16 & $12: 20: 13.4$ & 19.44 & 1.19 & 6 & \\
\hline 219 & 308.9 & 213.5 & 287.4 & 1.9 & 10:08:15.36 & $12: 14: 12.5$ & 18.29 & 1.36 & 5 & \\
\hline 220 & 309.6 & 208.3 & 293.8 & 2.1 & 10:08:16.98 & 12:13:57.4 & 19.08 & 1.25 & 4 & \\
\hline 221 & 312.5 & 331.7 & 273.3 & 2.1 & 10:08:16.89 & $12: 23: 05.2$ & 18.24 & 1.45 & 123 & \\
\hline 222 & 313.2 & 311.3 & 267.5 & 3.3 & $10: 08: 10.95$ & $12: 21: 56.8$ & 18.18 & 1.45 & 23 & \\
\hline 223 & 314.4 & 244.5 & 278.4 & 2.0 & 10:08:07.64 & $12: 16: 14.6$ & 18.81 & 1.33 & 6 & \\
\hline 224 & 314.5 & 214.5 & 288.2 & 2.0 & 10:08:14.84 & $12: 14: 10.9$ & 18.93 & 1.31 & 6 & \\
\hline 225 & 315.1 & 139.4 & 28.4 & 1.9 & 10:08:40.99 & 12:14:30.8 & 19.27 & 1.24 & 7 & \\
\hline 226 & 315.1 & 237.2 & 289.7 & 1.9 & 10:08:08.93 & $12: 15: 39.2$ & 18.40 & 1.41 & 1 & \\
\hline 227 & 318.2 & 284.8 & 276.4 & 1.9 & 10:08:06.01 & 12:19:51.3 & 19.22 & 1.24 & 7 & \\
\hline 228 & 318.4 & 298.2 & 491.2 & 2.1 & $10: 08: 07.85$ & $12: 21: 00.5$ & 18.67 & 1.32 & 5 & \\
\hline 229 & 318.5 & 184.4 & 278.8 & 2.0 & 10:08:25.32 & $12: 13: 12.5$ & 19.38 & 1.23 & 6 & \\
\hline 230 & 318.7 & 348.7 & 287.6 & 2.0 & $10: 08: 22.75$ & $12: 23: 42.6$ & 18.93 & 1.33 & 6 & \\
\hline 231 & 319.5 & 243.7 & 274.2 & 1.6 & 10:08:07.45 & 12:16:08.6 & 18.33 & 1.40 & 7 & \\
\hline 232 & 320.1 & 122.3 & 285.7 & 2.0 & $10: 08: 45.46$ & $12: 15: 38.9$ & 19.49 & 1.16 & 7 & \\
\hline 233 & 320.2 & 81.4 & 294.7 & 1.9 & 10:08:48.61 & $12: 19: 17.6$ & 19.49 & 1.19 & 7 & \\
\hline 234 & 320.4 & 275.3 & 290.2 & 2.0 & $10: 08: 05.23$ & $12: 18: 59.4$ & 19.26 & 1.17 & 6 & \\
\hline 235 & 320.6 & 216.3 & 286.2 & 1.8 & $10: 08: 14.06$ & $12: 14: 11.5$ & 19.45 & 1.13 & 7 & \\
\hline 236 & 321.2 & 77.0 & 298.0 & 2.1 & 10:08:48.36 & $12: 19: 42.2$ & 18.09 & 1.49 & 4 & \\
\hline 237 & 322.5 & 285.6 & 283.3 & 2.0 & 10:08:05.80 & $12: 19: 56.6$ & 18.91 & 1.33 & 2 & \\
\hline 238 & 324.7 & 280.0 & 282.6 & 1.8 & 10:08:05.18 & $12: 19: 26.4$ & 19.17 & 1.23 & 7 & \\
\hline 239 & 326.0 & 247.8 & 281.7 & 2.5 & 10:08:06.40 & $12: 16: 26.9$ & 18.19 & 1.47 & 1237 & \\
\hline 240 & 326.1 & 259.7 & 276.0 & 1.9 & 10:08:05.11 & $12: 17: 31.7$ & 18.78 & 1.30 & 6 & \\
\hline
\end{tabular}


Table 5-Continued

\begin{tabular}{|c|c|c|c|c|c|c|c|c|c|c|}
\hline ID $^{b}$ & $\begin{array}{l}R^{\mathrm{c}} \\
\left({ }^{\prime \prime}\right)\end{array}$ & $\begin{array}{r}P A^{\mathrm{c}} \\
(\mathrm{deg})\end{array}$ & $\begin{array}{c}v_{h} \\
(\mathrm{~km} / \mathrm{s})\end{array}$ & $\begin{array}{c}\sigma_{v_{h}} \\
(\mathrm{~km} / \mathrm{s})\end{array}$ & $\alpha_{J 2000}$ & $\delta_{J 2000}$ & I & $\mathrm{V}-\mathrm{I}$ & $\mathrm{SRC}^{\mathrm{d}}$ & $\begin{array}{l}\text { M98 } \\
\text { No. }\end{array}$ \\
\hline 241 & 326.5 & 263.6 & 280.5 & 1.9 & 10:08:04.86 & $12: 17: 53.6$ & 19.01 & 1.28 & 6 & \\
\hline 242 & 327.3 & 117.7 & 271.0 & 1.7 & 10:08:46.77 & $12: 15: 57.8$ & 18.54 & 1.38 & 7 & \\
\hline 243 & 329.0 & 169.7 & 292.7 & 2.0 & 10:08:31.00 & 12:13:06.3 & 19.06 & 1.38 & 6 & \\
\hline 244 & 329.4 & 220.5 & 89.7 & 2.0 & 10:08:12.42 & $12: 14: 19.3$ & 18.64 & 1.26 & 12 & \\
\hline 245 & 329.4 & 311.3 & 279.8 & 2.0 & 10:08:10.11 & $12: 22: 07.4$ & 19.56 & 1.20 & 7 & \\
\hline 246 & 330.6 & 237.1 & 278.3 & 1.7 & 10:08:08.06 & $12: 15: 30.4$ & 19.09 & 1.29 & 7 & \\
\hline 247 & 330.9 & 264.1 & 269.0 & 2.0 & $10: 08: 04.54$ & $12: 17: 56.1$ & 18.73 & 1.30 & 5 & \\
\hline 248 & 331.1 & 354.6 & 35.8 & 2.1 & $10: 08: 24.87$ & $12: 23: 59.6$ & 17.56 & 1.57 & 123 & \\
\hline 249 & 334.8 & 69.0 & 303.3 & 1.8 & $10: 08: 48.34$ & $12: 20: 29.7$ & 19.24 & 1.25 & 7 & \\
\hline 250 & 335.9 & 269.7 & 277.7 & 2.1 & 10:08:04.08 & 12:18:28.4 & 19.24 & 1.23 & 6 & \\
\hline 251 & 336.1 & 95.0 & 282.6 & 1.9 & $10: 08: 49.85$ & 12:18:00.8 & 18.68 & 1.32 & 4 & \\
\hline 252 & 337.1 & 37.0 & 287.1 & 2.0 & $10: 08: 40.85$ & $12: 22: 59.2$ & 18.90 & 1.29 & 5 & \\
\hline 253 & 338.1 & 235.4 & 289.2 & 1.9 & 10:08:08.01 & $12: 15: 18.0$ & 17.58 & 1.62 & 234 & \\
\hline 254 & 339.4 & 15.3 & -22.9 & 2.1 & 10:08:33.10 & $12: 23: 57.4$ & 19.56 & 1.18 & 1 & \\
\hline 255 & 339.5 & 272.2 & 276.2 & 1.9 & 10:08:03.85 & 12:18:43.2 & 19.44 & 1.25 & 7 & \\
\hline 256 & 340.1 & 95.5 & 270.8 & 1.9 & 10:08:50.10 & $12: 17: 57.3$ & 19.52 & 1.22 & 7 & \\
\hline 257 & 344.1 & 85.5 & 281.6 & 1.7 & 10:08:50.41 & $12: 18: 57.1$ & 18.66 & 1.32 & 7 & \\
\hline 258 & 347.0 & 113.0 & 282.8 & 2.0 & $10: 08: 48.79$ & $12: 16: 14.3$ & 19.03 & 1.30 & 5 & \\
\hline 259 & 347.0 & 291.3 & 277.7 & 1.8 & 10:08:04.94 & $12: 20: 36.1$ & 19.40 & 1.21 & 7 & \\
\hline 260 & 347.8 & 193.2 & 297.7 & 2.5 & 10:08:21.60 & $12: 12: 51.3$ & 18.46 & 1.36 & 26 & \\
\hline 261 & 349.3 & 79.7 & 292.6 & 2.1 & $10: 08: 50.45$ & $12: 19: 32.6$ & 18.98 & 1.30 & 6 & \\
\hline 262 & 350.7 & 277.0 & -12.8 & 1.8 & 10:08:03.25 & $12: 19: 12.9$ & 17.89 & 1.50 & 23 & \\
\hline 263 & 351.2 & 237.2 & 277.8 & 2.1 & 10:08:06.85 & $12: 15: 19.9$ & 19.52 & 1.24 & 6 & \\
\hline 264 & 353.0 & 320.4 & 16.1 & 1.9 & $10: 08: 11.65$ & $12: 23: 02.1$ & 17.59 & 1.72 & 2 & \\
\hline 265 & 353.5 & 58.2 & 276.2 & 2.0 & 10:08:47.50 & $12: 21: 36.4$ & 19.46 & 1.21 & 6 & \\
\hline 266 & 354.7 & 264.7 & 294.6 & 1.8 & 10:08:02.90 & $12: 17: 57.3$ & 19.24 & 1.23 & 7 & \\
\hline 267 & 355.0 & 77.7 & 282.6 & 1.8 & $10: 08: 50.67$ & $12: 19: 45.4$ & 19.33 & 1.22 & 7 & \\
\hline 268 & 356.5 & 208.0 & 288.8 & 2.0 & 10:08:15.58 & $12: 13: 15.2$ & 19.19 & 1.24 & 6 & \\
\hline 269 & 358.3 & 253.2 & 300.0 & 2.6 & 10:08:03.60 & $12: 16: 46.2$ & 18.64 & 1.23 & 235 & \\
\hline 270 & 359.4 & 49.8 & 260.2 & 2.1 & 10:08:45.73 & $12: 22: 22.1$ & 19.03 & 1.23 & 6 & \\
\hline
\end{tabular}


Table 5-Continued

\begin{tabular}{|c|c|c|c|c|c|c|c|c|c|c|}
\hline $\mathrm{ID}^{\mathrm{b}}$ & $\begin{array}{l}R^{\mathrm{c}} \\
\left({ }^{\prime \prime}\right)\end{array}$ & $\begin{array}{r}P A^{\mathrm{c}} \\
(\mathrm{deg})\end{array}$ & $\begin{array}{c}v_{h} \\
(\mathrm{~km} / \mathrm{s})\end{array}$ & $\begin{array}{c}\sigma_{v_{h}} \\
(\mathrm{~km} / \mathrm{s})\end{array}$ & $\alpha_{J 2000}$ & $\delta_{J 2000}$ & I & $\mathrm{V}-\mathrm{I}$ & $\mathrm{SRC}^{\mathrm{d}}$ & $\begin{array}{c}\text { M98 } \\
\text { No. }{ }^{\text {e }}\end{array}$ \\
\hline 271 & 361.2 & 258.0 & 263.9 & 1.8 & 10:08:02.90 & $12: 17: 14.6$ & 19.69 & 1.10 & 7 & \\
\hline 272 & 361.3 & 323.3 & 286.4 & 2.8 & $10: 08: 12.27$ & $12: 23: 19.7$ & 17.88 & 1.54 & 147 & \\
\hline 273 & 361.4 & 238.8 & 276.0 & 2.0 & 10:08:05.92 & $12: 15: 22.6$ & 17.65 & 1.69 & 5 & \\
\hline 274 & 362.6 & 120.8 & -22.9 & 2.1 & 10:08:48.26 & $12: 15: 24.5$ & 19.06 & 1.21 & 1 & \\
\hline 275 & 364.3 & 128.5 & 274.0 & 2.1 & 10:08:46.45 & 12:14:43.2 & 18.96 & 1.26 & 6 & \\
\hline 276 & 364.5 & 287.2 & 290.3 & 2.1 & 10:08:03.24 & $12: 20: 17.8$ & 18.31 & 1.42 & 23 & \\
\hline 277 & 366.0 & 61.0 & 276.0 & 1.8 & $10: 08: 48.85$ & $12: 21: 27.4$ & 18.93 & 1.26 & 7 & \\
\hline 278 & 370.6 & 334.4 & 277.0 & 2.1 & $10: 08: 16.07$ & $12: 24: 04.2$ & 19.14 & 1.20 & 23 & \\
\hline 279 & 370.8 & 48.9 & 266.3 & 2.3 & $10: 08: 46.08$ & $12: 22: 33.5$ & 18.20 & 1.47 & 12 & \\
\hline 280 & 372.0 & 301.9 & 300.1 & 1.7 & 10:08:05.45 & $12: 21: 46.6$ & 18.68 & 1.37 & 3 & \\
\hline 281 & 379.4 & 202.4 & -13.6 & 2.0 & 10:08:17.12 & 12:12:39.3 & 19.62 & 1.20 & 1 & \\
\hline 282 & 380.7 & 72.7 & 6.6 & 1.8 & 10:08:51.80 & $12: 20: 23.3$ & 19.20 & 1.26 & 7 & \\
\hline 283 & 382.1 & 60.9 & 278.5 & 2.0 & 10:08:49.78 & $12: 21: 36.0$ & 18.50 & 1.40 & 5 & \\
\hline 284 & 382.2 & 126.5 & 273.5 & 1.9 & 10:08:47.96 & 12:14:42.6 & 19.34 & 1.23 & 7 & \\
\hline 285 & 383.4 & 156.8 & 292.6 & 2.1 & 10:08:37.32 & $12: 12: 37.7$ & 19.11 & 1.28 & 6 & \\
\hline 286 & 388.0 & 278.2 & -0.5 & 1.9 & 10:08:00.79 & $12: 19: 25.2$ & 19.08 & 1.22 & 6 & \\
\hline 287 & 388.8 & 65.2 & 286.1 & 2.0 & 10:08:51.08 & $12: 21: 13.2$ & 19.59 & 1.17 & 7 & \\
\hline 288 & 392.0 & 249.4 & 272.5 & 1.8 & 10:08:01.96 & $12: 16: 12.2$ & 19.35 & 1.29 & 7 & \\
\hline 289 & 392.3 & 36.4 & 280.4 & 2.1 & 10:08:42.90 & $12: 23: 45.6$ & 18.17 & 1.51 & 234 & \\
\hline 290 & 393.2 & 252.0 & 260.1 & 2.0 & 10:08:01.49 & $12: 16: 28.3$ & 19.19 & 1.25 & 6 & \\
\hline 291 & 405.4 & 71.2 & -20.3 & 2.1 & 10:08:53.19 & $12: 20: 40.5$ & 19.13 & 1.27 & 1 & \\
\hline 292 & 407.7 & 266.3 & -14.1 & 2.1 & $10: 07: 59.24$ & 12:18:03.6 & 19.37 & 1.23 & 2 & \\
\hline 293 & 411.9 & 228.8 & 285.9 & 2.0 & 10:08:05.85 & $12: 13: 58.8$ & 18.72 & 1.30 & 1 & \\
\hline 294 & 413.9 & 294.2 & 279.1 & 2.1 & 10:08:01.24 & 12:21:19.8 & 19.33 & 1.20 & 2 & \\
\hline 295 & 415.1 & 260.0 & 307.2 & 2.8 & 10:07:59.11 & $12: 17: 17.6$ & 18.37 & 1.46 & 23 & \\
\hline 296 & 420.6 & 276.8 & -19.8 & 2.0 & $10: 07: 58.50$ & $12: 19: 19.6$ & 19.47 & 1.21 & 1 & \\
\hline 297 & 423.1 & 99.4 & -22.4 & 2.0 & 10:08:55.48 & $12: 17: 21.0$ & 18.96 & 1.26 & 1 & \\
\hline 298 & 423.9 & 123.4 & 276.1 & 2.1 & 10:08:51.15 & $12: 14: 36.8$ & 19.10 & 1.30 & 5 & \\
\hline 299 & 424.9 & 103.5 & 283.4 & 2.1 & 10:08:55.19 & $12: 16: 50.6$ & 19.16 & 1.24 & 1 & \\
\hline 300 & 425.7 & 77.6 & 280.4 & 2.1 & 10:08:55.37 & $12: 20: 01.4$ & 18.57 & 1.37 & 1 & \\
\hline
\end{tabular}


Table 5-Continued

\begin{tabular}{|c|c|c|c|c|c|c|c|c|c|c|}
\hline ID $^{b}$ & $\begin{array}{l}R^{\mathrm{c}} \\
\left({ }^{\prime \prime}\right)\end{array}$ & $\begin{array}{r}P A^{\mathrm{c}} \\
(\mathrm{deg})\end{array}$ & $\begin{array}{c}v_{h} \\
(\mathrm{~km} / \mathrm{s})\end{array}$ & $\begin{array}{c}\sigma_{v_{h}} \\
(\mathrm{~km} / \mathrm{s})\end{array}$ & $\alpha_{J 2000}$ & $\delta_{J 2000}$ & I & $\mathrm{V}-\mathrm{I}$ & $\mathrm{SRC}^{\mathrm{d}}$ & $\begin{array}{l}\text { M98 } \\
\text { No. }\end{array}$ \\
\hline 301 & 425.9 & 287.2 & 284.0 & 2.0 & 10:07:59.24 & $12: 20: 36.1$ & 18.54 & 1.39 & 2 & \\
\hline 302 & 426.5 & 291.9 & 260.9 & 5.0 & 10:07:59.99 & 12:21:09.0 & 18.73 & 1.30 & 46 & \\
\hline 303 & 427.0 & 155.8 & -11.8 & 2.0 & $10: 08: 38.96$ & 12:12:00.6 & 18.79 & 1.32 & 1 & \\
\hline 304 & 432.9 & 44.0 & 275.0 & 2.1 & 10:08:47.52 & $12: 23: 41.4$ & 19.04 & 1.26 & 5 & \\
\hline 305 & 434.7 & 112.7 & 288.9 & 1.9 & 10:08:54.36 & $12: 15: 42.2$ & 19.22 & 1.24 & 7 & \\
\hline 306 & 435.1 & 269.4 & 295.0 & 1.9 & 10:07:57.31 & $12: 18: 25.2$ & 18.44 & 1.39 & 5 & \\
\hline 307 & 442.8 & 105.4 & 290.9 & 1.9 & 10:08:56.12 & $12: 16: 32.1$ & 19.51 & 1.23 & 7 & \\
\hline 308 & 445.1 & 42.7 & 286.9 & 2.0 & 10:08:47.59 & $12: 23: 57.2$ & 19.03 & 1.25 & 23 & \\
\hline 309 & 449.9 & 54.5 & 279.7 & 2.0 & 10:08:51.99 & $12: 22: 51.4$ & 19.02 & 1.30 & 6 & \\
\hline 310 & 451.0 & 98.9 & 298.3 & 1.9 & 10:08:57.40 & $12: 17: 19.9$ & 19.27 & 1.24 & 7 & \\
\hline 311 & 458.7 & 55.9 & 97.0 & 2.1 & 10:08:52.93 & $12: 22: 47.0$ & 18.77 & 1.18 & 15 & \\
\hline 312 & 463.2 & 276.1 & 284.1 & 3.7 & $10: 07: 55.57$ & $12: 19: 19.3$ & 19.06 & 1.27 & 23 & \\
\hline 313 & 464.4 & 306.5 & 273.1 & 3.1 & 10:08:01.53 & $12: 23: 06.4$ & 19.09 & 1.31 & 23 & \\
\hline 314 & 465.0 & 89.2 & 276.9 & 2.0 & 10:08:58.73 & $12: 18: 36.1$ & 18.48 & 1.40 & 1 & \\
\hline 315 & 466.8 & 31.5 & 276.3 & 1.7 & 10:08:43.63 & $12: 25: 08.2$ & 18.66 & 1.33 & 7 & \\
\hline 316 & 469.1 & 282.4 & 296.8 & 2.1 & 10:07:55.74 & $12: 20: 10.9$ & 19.15 & 1.27 & 4 & \\
\hline 317 & 470.5 & 49.4 & 292.9 & 1.8 & 10:08:51.40 & $12: 23: 35.9$ & 19.21 & 1.24 & 7 & \\
\hline 318 & 470.5 & 202.0 & 29.9 & 1.9 & 10:08:14.97 & 12:11:13.8 & 18.50 & 1.30 & 6 & \\
\hline 319 & 471.2 & 302.1 & 273.2 & 2.0 & $10: 07: 59.76$ & $12: 22: 40.4$ & 18.69 & 1.35 & 467 & \\
\hline 320 & 475.6 & 127.1 & 266.1 & 2.0 & $10: 08: 52.88$ & 12:13:43.1 & 19.41 & 1.21 & 3 & \\
\hline 321 & 476.4 & 77.4 & 274.0 & 2.0 & 10:08:58.73 & $12: 20: 13.7$ & 19.04 & 1.20 & 6 & \\
\hline 322 & 481.9 & 313.6 & 295.2 & 7.6 & 10:08:03.19 & $12: 24: 02.4$ & 18.88 & 1.33 & 56 & \\
\hline 323 & 494.9 & 56.8 & 279.8 & 2.0 & $10: 08: 55.27$ & $12: 23: 00.8$ & 18.59 & 1.38 & 1 & \\
\hline 324 & 496.7 & 122.7 & 281.7 & 2.6 & $10: 08: 55.51$ & $12: 14: 01.5$ & 17.93 & 1.42 & 1234 & \\
\hline 325 & 499.3 & 242.9 & 265.7 & 2.0 & 10:07:56.68 & $12: 14: 42.3$ & 19.16 & 1.22 & 7 & \\
\hline 326 & 500.4 & 61.2 & 273.4 & 1.9 & 10:08:56.92 & $12: 22: 31.3$ & 18.60 & 1.36 & 2 & \\
\hline 327 & 501.5 & 318.1 & -16.4 & 2.0 & 10:08:04.13 & $12: 24: 43.1$ & 19.44 & 1.07 & 1 & \\
\hline 328 & 505.5 & 227.0 & 274.2 & 1.9 & 10:08:01.80 & $12: 12: 44.9$ & 19.63 & 1.12 & 7 & \\
\hline 329 & 511.4 & 282.5 & 295.1 & 2.1 & 10:07:52.93 & $12: 20: 20.6$ & 19.08 & 1.29 & 5 & \\
\hline 330 & 513.3 & 205.6 & 134.4 & 1.9 & 10:08:11.85 & $12: 10: 47.2$ & 18.87 & 1.25 & 6 & \\
\hline
\end{tabular}


Table 5-Continued

\begin{tabular}{|c|c|c|c|c|c|c|c|c|c|c|}
\hline $\mathrm{ID}^{\mathrm{b}}$ & $\begin{array}{l}R^{\mathrm{c}} \\
\left({ }^{\prime \prime}\right)\end{array}$ & $\begin{array}{r}P A^{\mathrm{c}} \\
(\operatorname{deg})\end{array}$ & $\begin{array}{c}v_{h} \\
(\mathrm{~km} / \mathrm{s})\end{array}$ & $\begin{array}{c}\sigma_{v_{h}} \\
(\mathrm{~km} / \mathrm{s})\end{array}$ & $\alpha_{J 2000}$ & $\delta_{J 2000}$ & I & $\mathrm{V}-\mathrm{I}$ & $\mathrm{SRC}^{\mathrm{d}}$ & $\begin{array}{l}\text { M98 } \\
\text { No. }^{\text {e }}\end{array}$ \\
\hline 331 & 515.3 & 64.9 & -19.3 & 2.0 & 10:08:58.86 & $12: 22: 08.1$ & 19.50 & 1.20 & 1 & \\
\hline 332 & 516.7 & 26.5 & 279.6 & 2.1 & 10:08:42.73 & $12: 26: 12.4$ & 19.47 & 1.20 & 1 & \\
\hline 333 & 520.2 & 108.1 & 266.2 & 2.5 & 10:09:00.74 & $12: 15: 48.6$ & 18.58 & 1.35 & 24 & \\
\hline 334 & 527.1 & 105.1 & 120.2 & 2.0 & 10:09:01.72 & $12: 16: 12.4$ & 18.53 & 1.24 & 34 & \\
\hline 335 & 529.2 & 149.3 & 21.6 & 5.7 & 10:08:45.41 & $12: 10: 54.8$ & 17.07 & 1.89 & 12 & \\
\hline 336 & 540.9 & 69.4 & 272.1 & 2.5 & 10:09:01.55 & $12: 21: 40.4$ & 18.26 & 1.39 & 12 & \\
\hline 337 & 541.3 & 151.6 & 283.7 & 2.0 & $10: 08: 44.57$ & $12: 10: 33.9$ & 18.50 & 1.25 & 3 & \\
\hline 338 & 544.9 & 58.7 & 297.1 & 1.8 & 10:08:58.78 & $12: 23: 13.0$ & 18.71 & 1.30 & 7 & \\
\hline 339 & 547.8 & 255.9 & 294.5 & 2.0 & 10:07:50.76 & $12: 16: 16.1$ & 18.74 & 1.32 & 2 & \\
\hline 340 & 553.9 & 80.7 & 286.6 & 1.8 & 10:09:04.30 & 12:19:59.3 & 18.90 & 1.26 & 7 & \\
\hline 341 & 558.1 & 255.5 & 282.3 & 1.8 & 10:07:50.13 & $12: 16: 10.3$ & 19.30 & 1.26 & 7 & \\
\hline 342 & 561.2 & 76.0 & 284.8 & 2.7 & 10:09:04.16 & $12: 20: 45.8$ & 18.55 & 1.35 & 23 & \\
\hline 343 & 566.3 & 81.2 & 285.0 & 3.1 & 10:09:05.19 & $12: 19: 56.4$ & 18.26 & 1.42 & 14 & \\
\hline 344 & 568.4 & 294.6 & 273.1 & 5.0 & $10: 07: 51.73$ & $12: 22: 26.5$ & 18.90 & 1.38 & 67 & \\
\hline 345 & 568.8 & 79.4 & 283.1 & 2.0 & 10:09:05.16 & 12:20:14.1 & 18.43 & 1.38 & 3 & \\
\hline 346 & 588.8 & 261.7 & 52.4 & 1.8 & $10: 07: 47.25$ & $12: 17: 04.5$ & 17.73 & 1.55 & 234 & \\
\hline 347 & 593.6 & 89.2 & 286.4 & 2.0 & 10:09:07.50 & $12: 18: 38.2$ & 18.29 & 1.43 & 234 & \\
\hline 348 & 608.2 & 115.6 & 274.0 & 2.1 & 10:09:04.43 & $12: 14: 07.4$ & 19.44 & 1.23 & 3 & \\
\hline 349 & 610.0 & 283.8 & 294.7 & 1.7 & $10: 07: 46.58$ & $12: 20: 55.8$ & 18.26 & 1.49 & 3 & \\
\hline 350 & 631.0 & 297.9 & 296.4 & 1.9 & 10:07:48.94 & $12: 23: 25.3$ & 19.04 & 1.30 & 36 & \\
\hline 351 & 632.9 & 343.4 & 91.7 & 2.0 & $10: 08: 14.67$ & $12: 28: 36.6$ & 17.35 & 1.74 & 12 & \\
\hline 352 & 638.6 & 276.6 & 271.3 & 1.9 & 10:07:43.71 & $12: 19: 42.8$ & 19.30 & 1.23 & 3 & \\
\hline 353 & 648.9 & 11.8 & 96.1 & 2.2 & 10:08:36.07 & $12: 29: 05.2$ & 17.91 & 1.61 & 24 & \\
\hline 354 & 650.5 & 293.2 & 295.8 & 2.2 & 10:07:46.19 & $12: 22: 46.0$ & 18.82 & 1.34 & 3567 & \\
\hline 355 & 657.2 & 54.0 & -51.3 & 2.2 & 10:09:03.28 & $12: 24: 56.4$ & 18.18 & 1.52 & 36 & \\
\hline 356 & 688.6 & 95.6 & 294.8 & 2.8 & 10:09:13.76 & $12: 17: 22.6$ & 18.58 & 1.37 & 237 & \\
\hline 357 & 692.1 & 279.5 & 276.3 & 3.0 & $10: 07: 40.41$ & $12: 20: 23.5$ & 18.75 & 1.27 & 3467 & \\
\hline 358 & 695.3 & 145.6 & -21.9 & 3.1 & 10:08:53.78 & 12:08:56.1 & 17.72 & 1.58 & 123 & \\
\hline 359 & 700.6 & 211.3 & -21.9 & 2.3 & 10:08:02.15 & 12:08:31.6 & 17.31 & 1.84 & 124567 & \\
\hline 360 & 702.5 & 262.2 & 294.8 & 2.8 & 10:07:39.51 & $12: 16: 54.6$ & 18.92 & 1.22 & 346 & \\
\hline
\end{tabular}


Table 5-Continued

\begin{tabular}{|c|c|c|c|c|c|c|c|c|c|c|}
\hline $\mathrm{ID}^{\mathrm{b}}$ & $\begin{array}{l}R^{\mathrm{c}} \\
\left({ }^{\prime \prime}\right)\end{array}$ & $\begin{array}{r}P A^{\mathrm{c}} \\
(\mathrm{deg})\end{array}$ & $\begin{array}{c}v_{h} \\
(\mathrm{~km} / \mathrm{s})\end{array}$ & $\begin{array}{c}\sigma_{v_{h}} \\
(\mathrm{~km} / \mathrm{s})\end{array}$ & $\alpha_{J 2000}$ & $\delta_{J 2000}$ & I & $\mathrm{V}-\mathrm{I}$ & $\mathrm{SRC}^{\mathrm{d}}$ & $\begin{array}{l}\text { M98 } \\
\text { No. }^{\mathrm{e}}\end{array}$ \\
\hline 361 & 702.7 & 89.6 & 260.8 & 2.0 & 10:09:14.95 & $12: 18: 34.3$ & 18.47 & 1.38 & 13 & \\
\hline 362 & 704.2 & 60.0 & 268.0 & 2.0 & 10:09:08.65 & $12: 24: 21.4$ & 18.53 & 1.40 & 3 & \\
\hline 363 & 705.8 & 227.7 & -13.4 & 1.9 & 10:07:51.39 & $12: 10: 34.9$ & 17.36 & 1.83 & 1234 & \\
\hline 364 & 719.5 & 95.7 & 274.7 & 2.0 & 10:09:15.85 & $12: 17: 18.2$ & 18.60 & 1.40 & 5 & \\
\hline 365 & 720.0 & 266.4 & 284.0 & 1.8 & 10:07:37.97 & $12: 17: 44.4$ & 18.72 & 1.32 & 3 & \\
\hline 366 & 734.3 & 103.0 & 272.8 & 3.0 & 10:09:15.82 & $12: 15: 45.1$ & 18.94 & 1.27 & 13 & \\
\hline 367 & 735.6 & 55.2 & 53.8 & 2.8 & 10:09:08.23 & $12: 25: 29.7$ & 17.87 & 1.50 & 45 & \\
\hline 368 & 750.7 & 244.2 & 281.0 & 2.1 & 10:07:40.89 & 12:13:03.3 & 18.40 & 1.44 & 23456 & \\
\hline 369 & 760.8 & 228.2 & 65.3 & 1.9 & 10:07:48.34 & $12: 10: 02.4$ & 19.91 & 1.01 & 7 & \\
\hline 370 & 770.8 & 141.9 & -12.3 & 2.0 & 10:08:59.47 & $12: 08: 23.7$ & 19.12 & 1.22 & 1 & \\
\hline 371 & 772.6 & 69.5 & 103.8 & 2.0 & 10:09:16.41 & $12: 22: 59.8$ & 17.19 & 1.75 & 234 & \\
\hline 372 & 789.2 & 77.4 & 274.8 & 2.0 & 10:09:19.56 & $12: 21: 21.9$ & 18.99 & 1.20 & 3 & \\
\hline 373 & 792.4 & 230.3 & 40.0 & 2.1 & 10:07:45.41 & 12:10:03.9 & 18.04 & 1.58 & 234 & \\
\hline 374 & 795.4 & 74.8 & 290.2 & 3.2 & 10:09:19.40 & $12: 21: 57.7$ & 18.30 & 1.43 & 34 & \\
\hline 375 & 823.1 & 92.2 & 275.0 & 2.0 & 10:09:23.12 & $12: 17: 57.9$ & 18.81 & 1.36 & 4 & \\
\hline 376 & 825.5 & 239.3 & 268.2 & 3.4 & 10:07:38.58 & $12: 11: 28.5$ & 19.25 & 1.24 & 347 & \\
\hline 377 & 839.4 & 245.6 & 282.3 & 2.2 & 10:07:34.84 & 12:12:43.6 & 19.32 & 1.20 & 37 & \\
\hline 378 & 848.5 & 282.4 & 298.0 & 2.0 & 10:07:30.45 & $12: 21: 32.4$ & 19.42 & 1.21 & 357 & \\
\hline 379 & 864.7 & 44.5 & 2.1 & 3.5 & 10:09:08.37 & $12: 28: 46.7$ & 17.84 & 1.53 & 12345 & \\
\hline 380 & 883.1 & 45.3 & 25.1 & 2.0 & 10:09:09.88 & $12: 28: 50.6$ & 18.95 & 1.29 & 6 & \\
\hline 381 & 940.9 & 136.3 & 4.5 & 2.0 & 10:09:11.35 & 12:07:09.9 & 19.42 & 1.14 & 2 & \\
\hline 382 & 952.6 & 246.2 & -55.8 & 1.8 & 10:07:27.56 & $12: 12: 04.9$ & 18.30 & 1.41 & 3 & \\
\hline 383 & 957.3 & 45.6 & 84.4 & 1.9 & 10:09:13.67 & $12: 29: 40.1$ & 19.31 & 1.19 & 7 & \\
\hline 384 & 968.5 & 248.5 & 26.9 & 2.5 & 10:07:25.54 & $12: 12: 34.5$ & 18.62 & 1.40 & 356 & \\
\hline 385 & 1034.6 & 30.4 & 140.2 & 5.5 & 10:09:02.73 & $12: 33: 22.4$ & 18.77 & 1.31 & 35 & \\
\hline 386 & 1056.1 & 244.8 & 18.8 & 2.0 & 10:07:21.82 & 12:11:00.1 & 19.32 & 1.20 & 45 & \\
\hline 387 & 1169.0 & 17.2 & -5.6 & 2.7 & 10:08:50.58 & $12: 37: 06.8$ & 19.00 & 1.26 & 456 & \\
\hline
\end{tabular}


aStars with heliocentric velocities (column 4) in the range $250-320 \mathrm{~km} / \mathrm{s}$ are treated as members in our analysis. Star 30 in this table was not identified in our new photometry because if fell in a gap between 90Prime CCDs. Its equatorial coordinates (in boldface) are taken directly from M98 and may be offset by 2-3 arcsec relative to the coordinates of all other stars in the Table.

${ }^{b}$ Star identification number, ordered by increasing radial distance from the center of Leo I ( $R$, column 2). IDs in italics are possible 'false positives' where we may have obtained the velocity of the sky at the time of observation rather than an actual target star (see Figure9 and Section 3.2). Star IDs in boldface denote possible binary stars based on velocity variability (see Figure 11 and Sections 3.4 and 4.1).

${ }^{\mathrm{c}} R$ (distance from center in arcsec) and $P A$ (position angle relative to center in degrees with $P A($ North $)=0 \mathrm{deg}$ and $P A($ East $)=90 \mathrm{deg})$ are defined relative to the adopted center of Leo I: $\left(\alpha_{c, J 2000}\right.$, delta $\left._{c, 2000}\right)=(10: 08: 27,+12: 18: 30)$ (Mateo 1998). This center position differs somewhat from the one used by M98 and from the center coordinate given by IH95.

${ }^{\mathrm{d}}$ The sources of the velocities used to estimate the mean velocity listed in this table are coded as: $1=$ Leo I/c1, $2=$ Leo I/c2; $3=$ Leo I/c3; $4=$ Leo I/c4; $5=$ Leo I/c5; $6=$ Leo I/c6; $7=$ Leo I/c7; $8=$ M98. See Table 1 for details about each of these configurations.

eStar number from M98. 
Table 6. Summary of Adopted and Derived Properties of Leo I

\begin{tabular}{|c|c|c|c|}
\hline Quantity & Value & Units & Reference $^{\mathrm{a}}$ \\
\hline \multicolumn{4}{|c|}{ Adopted Parameters ${ }^{\mathrm{b}}$} \\
\hline$\alpha_{2000}($ center $)$ & 100827.0 & & M98R \\
\hline$\delta_{2000}($ center $)$ & +121830 & & M98R \\
\hline Distance & $255 \pm 23$ & $\mathrm{kpc}$ & C99; H01; B04 \\
\hline$R_{\text {core }}$ & $3.3 \pm 0.3$ & $\operatorname{arcmin}$ & IH95 \\
\hline$R_{\text {tidal }}$ & $12.6 \pm 1.5$ & $\operatorname{arcmin}$ & IH95 \\
\hline$R_{\text {core }}$ & $245 \pm 35$ & $\mathrm{pc}$ & IH95+Dist \\
\hline$R_{t i d a l}$ & $935 \pm 140$ & $\mathrm{pc}$ & IH95+Dist \\
\hline$M_{V, t o t}$ & $-12.0 \pm 0.3$ & mag & IH95+Dist \\
\hline$L_{V, t o t}$ & $5.6 \pm 1.8 \times 10^{6}$ & $L_{\odot}$ & IH95+Dist \\
\hline$\Sigma_{0}$ & $22.6 \pm 0.3$ & $\mathrm{~V}$ mag $\operatorname{arcsec}^{-2}$ & IH95 \\
\hline$S_{0}$ & $40 \pm 12$ & $L_{\odot} \mathrm{pc}^{-2}$ & IH95+Dist \\
\hline$I_{0}$ & $0.07 \pm 0.02$ & $L_{\odot} \mathrm{pc}^{-3}$ & IH95+Dist \\
\hline$\left(M / L_{V}\right)_{\text {baryons }}$ & 0.3-0.7 & $M_{\odot} / L_{V, \odot}$ & M98; Sec 4.2 \\
\hline$\rho_{0, \text { baryons }}$ & $0.02-0.05$ & $M_{\odot} \mathrm{pc}^{-3}$ & M98; Sec 4.2 \\
\hline
\end{tabular}

Derived Global Kinematic/Structural Parameters ${ }^{\mathrm{c}}$

$\begin{array}{lccl}\left\langle v_{\text {helio }}\right\rangle & 282.9 \pm 0.5 & \mathrm{~km} / \mathrm{s} & \text { Sec } 4.3 .1 \\ \left\langle v_{G S}\right\rangle & 174.9 \pm 0.5 & \mathrm{~km} / \mathrm{s} & \text { Sec } 4.3 .1 \\ \sigma_{v} & 9.2 \pm 0.4 & \mathrm{~km} / \mathrm{s} & \text { Sec } 4.3 .1 \\ e_{R=0^{\prime}} & 0.21 \pm 0.03 & (1-b / a) & \text { IH95; Fig 17 } \\ e_{R=12^{\prime}} & 0.34 \pm 0.02 & (1-b / a) & \text { Fig 17, Sec 4.3.2 } \\ P A_{R=0^{\prime}} & 79 \pm 3 & \text { degrees } & \text { IH95; Fig 17 } \\ P A_{R=12^{\prime}} & 84 \pm 3 & \text { degrees } & \text { Fig 17 Sec 4.3.2 }\end{array}$


Table 6-Continued

\begin{tabular}{|c|c|c|c|}
\hline Quantity & Value & Units & Reference $^{\mathrm{a}}$ \\
\hline \multicolumn{4}{|c|}{ Equilibrium Dynamical Model Results } \\
\hline \multicolumn{4}{|c|}{ Isothermal Model ${ }^{\mathrm{d}}$} \\
\hline $\begin{array}{l}\rho_{0} \\
\left(M / L_{V}\right)_{0}=\rho_{0} / I_{0} \\
M(R \leq 1040 \mathrm{pc}) \\
M / L_{V}(R \leq 1040 \mathrm{pc}) \\
\chi_{r}^{2}\end{array}$ & $\begin{array}{c}0.23 \pm 0.04 \\
3.3 \pm 1.1 \\
5.2 \pm 1.2 \times 10^{7} \\
9.3 \pm 4.0 \\
0.51\end{array}$ & $\begin{array}{l}M_{\odot} \mathrm{pc}^{-3} \\
M_{\odot} / L_{V, \odot} \\
\quad M_{\odot} \\
M_{\odot} / L_{V, \odot}\end{array}$ & $\begin{array}{l}\text { Sec } 4.2 \\
\text { Sec } 4.2 \\
\text { Sec } 4.2 \\
\text { Sec } 4.2 \\
\text { Sec } 4.2 ; \text { Fig } 12\end{array}$ \\
\hline \multicolumn{4}{|c|}{ Sersic+NFW Model ${ }^{\mathrm{e}}$} \\
\hline$\rho_{0, \text { Sersic }}$ & $0.04 \pm 0.01$ & $M_{\odot} \mathrm{pc}^{-3}$ & Sec 4.2; Fig 17 \\
\hline$r_{\text {Sersic }}$ & $370 \pm 30$ & $\mathrm{pc}$ & Sec 4.2; Fig 17 \\
\hline$m_{S}$ & $0.6 \pm 0.1$ & & Sec $4.2 ;$ Fig 17 \\
\hline$R_{v i r}$ & $18.3 \pm 2.7$ & $\mathrm{kpc}$ & Sec 4.2 \\
\hline$M_{v i r} \equiv M\left(R_{v i r}\right)$ & $7 \pm 1 \times 10^{8}$ & $M_{\odot}$ & Sec 4.2 \\
\hline$R_{s} \equiv R_{v i r} / 20$ & $916 \pm 140$ & $\mathrm{pc}$ & Sec 4.2 \\
\hline$\eta \equiv M_{v i r} / M_{v i s}$ & $129 \pm 45$ & & Sec 4.2 \\
\hline$M_{v i r} / L_{V}$ & $125 \pm 44$ & $M_{\odot} / L_{V, \odot}$ & Sec 4.2 \\
\hline$M(R \leq 1040 \mathrm{pc})$ & $8.1 \pm 2.0 \times 10^{7}$ & $M_{\odot}$ & Sec 4.2 \\
\hline$M / L_{V}(R \leq 1040 \mathrm{pc})$ & $14.4 \pm 5.8$ & $M_{\odot} / L_{V, \odot}$ & $\operatorname{Sec} 4.2$ \\
\hline$\beta(95 \%$ c.l. $)$ & $-1.5_{-1.7}^{+1.1}$ & & $\operatorname{Sec} 4.2 ; \operatorname{Fig} 12$ \\
\hline$\chi_{r}^{2}(\beta=-1.5)$ & 0.74 & & Sec 4.2 ; Fig 12 \\
\hline
\end{tabular}


a References: M98R = Mateo 1998; C99 = Caputo et al. 1999; H01 $=$ Held et al. 2001; B04 = Bellazzini et al. 2004; IH95 = Irwin and Hatzidimitriou 1995; IH95+Dist refers to IH95 results adjusted for the distance used in this paper; M98 = Mateo et al. 1998. Figures and Section numbers refer to this paper.

${ }^{\mathrm{b}} \Sigma_{0}$ and $S_{0}$ are the central surface brightness of Leo I in astronomical and physical units, respectively; $I_{0}$ is the central luminosity density, and $\rho_{0, \text { baryons }}$ is the central mass density for the assumed bary-

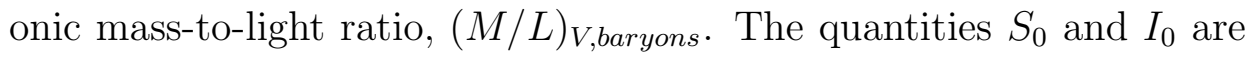
calculated as described in M98.

c'GS' refers to the Galactostationary reference frame (see Section 4.3 .1 ) based on an LSR motion of $220 \mathrm{~km} / \mathrm{s}$ toward $(l, b)=(90,0)$, and a solar peculiar motion relative to the LSR of $16.6 \mathrm{~km} / \mathrm{s}$ toward $(l, b)=(53,25)$. The position angles $(P A)$ and isophotal ellipticities (e) are defined in Figure 17. See Section 4.3.1 for details.

${ }^{\mathrm{d}} \rho_{0}$ is the total central mass density (DM + baryons).

eThe Sersic index, $m_{S}$, and Sersic radius, $r_{\text {Sersic }}$ are defined as $I_{\text {Sersic }}=I_{0} \exp \left[-\left(r / r_{\text {Sersic }}\right)^{1 / m_{S}}\right]$. Values of $r_{\text {Sersic }}$ and $m_{S}$ were derived from fitting our stellar density profile for Leo I (Figure 17). The anisotropy parameter, $\beta$ is defined such that $\beta=0$ corresponds to an isotropic distribution, $\beta=+1$ is a fully radially anisotropic distribution, and $\beta=-\infty$ is a fully tangentially anisotropic distribution (Binney and Tremaine 1987). Following Łokas, we set the virial radius, $R_{\text {vir }}$, such that $\rho_{N F W}\left(R_{\text {vir }}\right)=200 \rho_{\text {crit }}$. 
Table 7: Population Segregation in Leo I

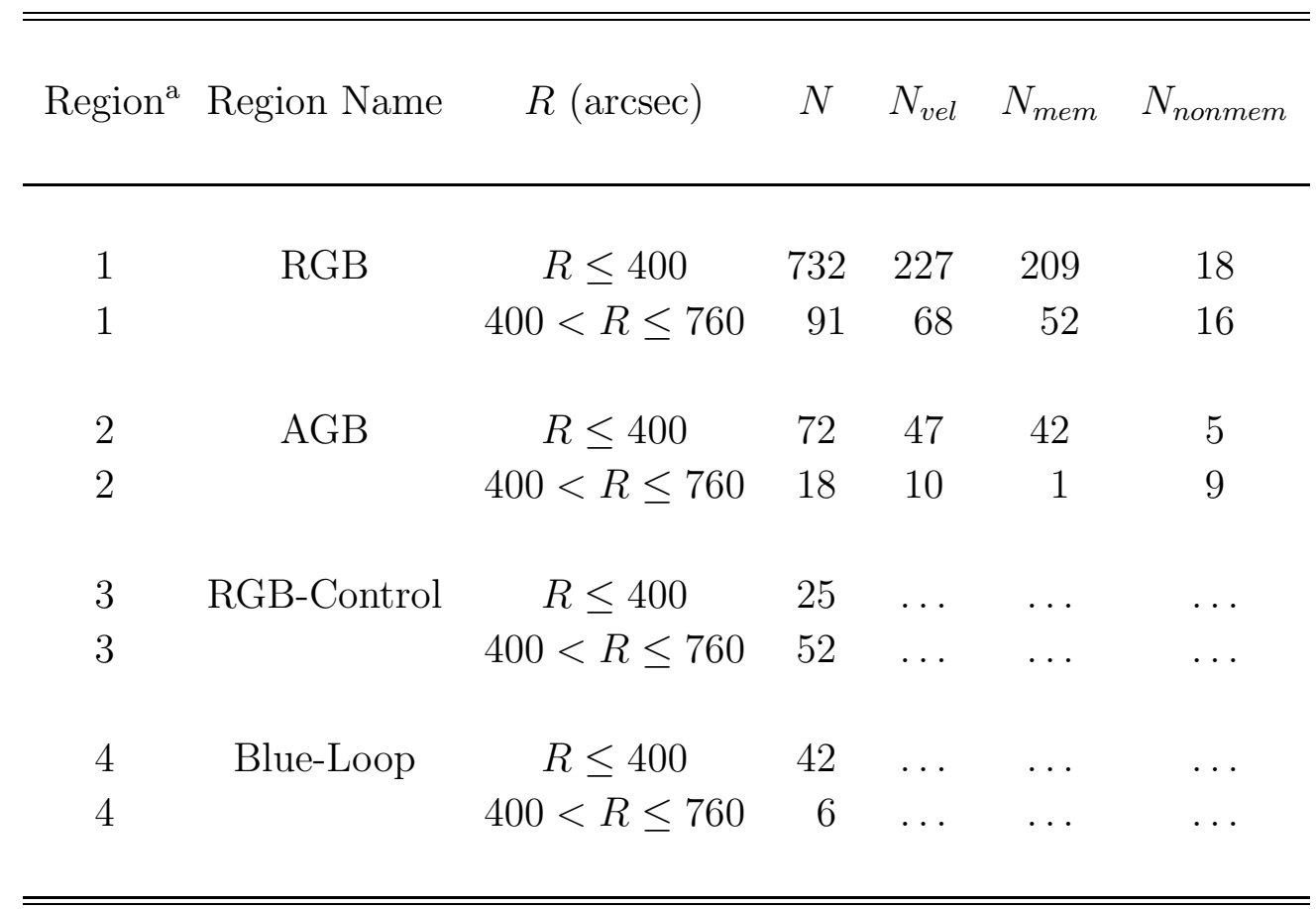

${ }^{a}$ The column labeled 'Region' corresponds to the regions of the Leo I CMD identified in Figure 23, 'Region Name' identifies the principal component in each region: RGB = Red Giant Branch stars; AGB = Asymptotic Giant Branch stars; RGB-Control = Field stars in a similar magnitude range as the RGB stars; Blue-Loop = intermediate-age core He burning stars in a blue phase of their post-main sequence evolution (Dohm-Palmer and Skillman 2002). The other columns in this table are $R$, the radial extent fro the center of Leo I, $N$ the total number of stars in a given region, $N_{\text {vel }}$ the number of stars in a region with measured velocities, and $N_{\text {mem }}, N_{\text {nonmem }}$, the number of kinematic members and non-members in a given region, respectively. The sole AGB star with $R>400 \operatorname{arcsec}$ is located at $R=497 \operatorname{arcsec}($ star 324 in Table 5). 
Table 8: Representative Orbital Parameters for Leo I ${ }^{\text {a }}$

\begin{tabular}{|c|c|c|c|c|c|c|c|c|}
\hline $\begin{array}{l}V_{G S, \text { rad }} \\
(\mathrm{km} / \mathrm{s})\end{array}$ & $\begin{array}{l}V_{G S, \tan } \\
(\mathrm{km} / \mathrm{s})\end{array}$ & $\begin{array}{l}R_{\text {peri }} \\
(\mathrm{kpc})\end{array}$ & $\begin{array}{l}R_{\text {apo }} \\
(\mathrm{Gyr})\end{array}$ & $\begin{array}{l}T_{\text {peri }} \\
(\mathrm{kpc})\end{array}$ & $P_{o r b}$ & $\begin{array}{c}e \\
(\mathrm{Gyr})\end{array}$ & $\begin{array}{c}\mu_{\alpha} \\
(\operatorname{marcsec} / \text { cent })\end{array}$ & $\begin{array}{c}\mu_{\delta} \\
(\operatorname{marcsec} / \text { cent })\end{array}$ \\
\hline 181 & 5 & 2.5 & 399 & 0.91 & 5.4 & 0.99 & -4.1 & -16.3 \\
\hline 181 & 13 & 6.1 & 399 & 0.91 & 5.4 & 0.97 & -3.4 & -15.9 \\
\hline 180 & 24 & 11.5 & 400 & 0.92 & 5.4 & 0.94 & -2.6 & -15.4 \\
\hline 180 & 34 & 16.9 & 402 & 0.93 & 5.5 & 0.92 & -1.9 & -15.0 \\
\hline 180 & 44 & 23.5 & 404 & 0.94 & 5.6 & 0.89 & -1.1 & -14.6 \\
\hline 180 & 54 & 30.3 & 408 & 0.95 & 5.7 & 0.86 & -0.4 & -14.1 \\
\hline 177 & 207 & 159 & 656 & 0.80 & 10.2 & 0.61 & +10.8 & -7.7 \\
\hline
\end{tabular}

${ }^{a}$ These results are derived for model orbits using the Milky Way gravitational potential given by Johnston et al. (1995), adopting an asymptotic halo circular velocity of $190 \mathrm{~km} / \mathrm{s}$. The results here were calculated for an orbit with an adopted pole of $(l, b)=(130,0)$, slightly different than the value used to produce the orbit shown in Figure 13. The column headers are: $V_{G S, r a d}=$ Galactostationary radial velocity, $V_{G S, t a n}=$ Galactostationary tangential velocity, $R_{\text {peri }}=$ perigalactic distance, $T_{\text {peri }}=$ time of last perigalactic passage, measured backward in time from the present, $R_{\text {apo }}=$ apogalactic distance, $P_{\text {orb }}=$ radial orbital period, and $e=\left(1-\left(R_{\text {peri }} / R_{\text {apo }}\right) /\left(1+\left(R_{\text {peri }} / R_{\text {apo }}\right)\right)\right.$ is the orbital eccentricity. The columns labeled $\mu_{\alpha}$ and $\mu_{\delta}$ are the observed heliocentric proper motions (in RA and Dec, respectively) in units of marcsec/century for the current epoch and assuming Leo I is at its present location and distance. These orbital parameters are essentially independent of the mass of Leo I for the range of masses explored in Figure 25. 


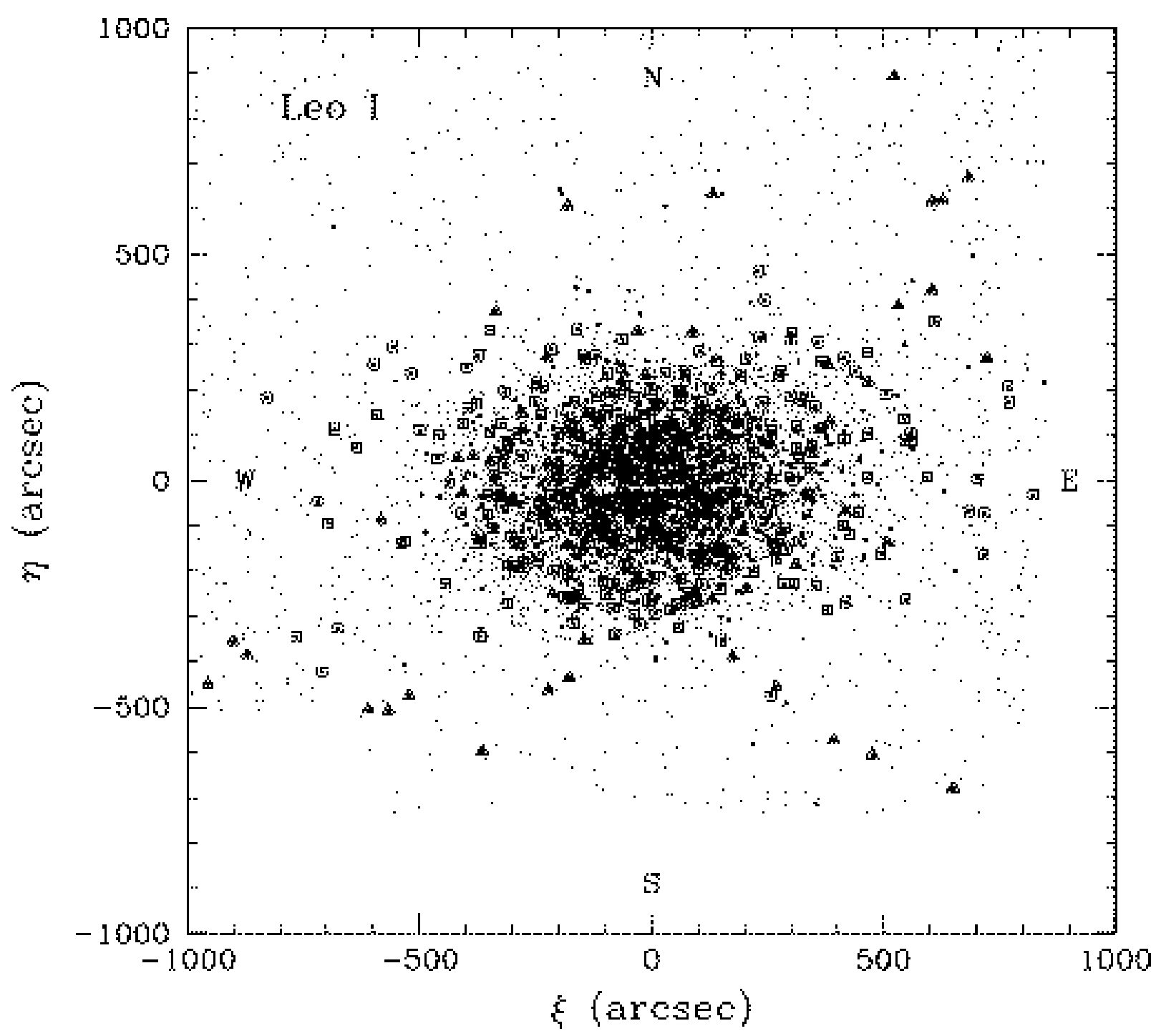

Fig. 1. - The spatial distribution of stars in our Leo I color-magnitude diagram (Figure 2). Candidate Leo I red giants are denoted as small filled black squares (see Figure 2 for the candidate selection definition). Stars with MMT/Hectochelle spectroscopy from 2005-2007 are shown as open squares (kinematic members) and open triangles (kinematic non-members). Open circles denote stars also observed spectroscopically with HIRES on Keck by M98. The standard coordinates adopt the center of Leo I at $(\alpha, \delta)_{2000.0}=(10: 08: 27,+12: 18: 30)$ (Mateo 1998) as the tangent point. Each Hectochelle field (see Table 1) spans an area much larger than the full extent of the region plotted here. 


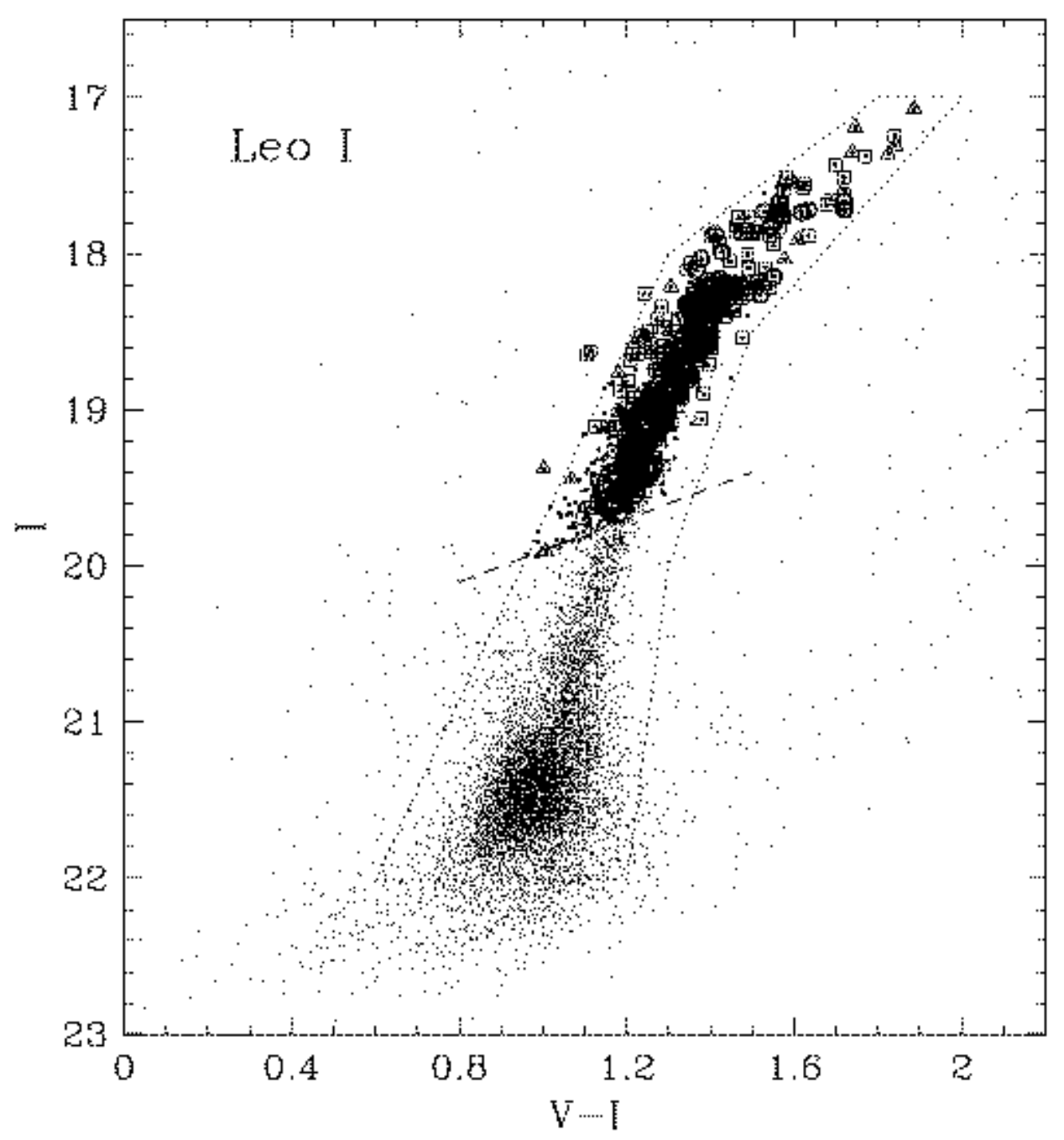

Fig. 2. - The calibrated Leo I color-magnitude diagram based on our new 90Prime photometry. The selection region for spectroscopic candidates can be discerned from the distribution of the small filled squares, while open squares and open triangles denote stars we have observed with Hectochelle. Open circles denote stars from the Keck/HIRES sample of M98. The dotted lines enclose the stars used to measure the structural parameters of Leo I (Section 4.3.2). Though difficult to see here because of crowding, about $75 \%$ of the stars we have observed with Hectochelle lie along the RGB, below the base of the extended AGB at $I \sim 18.1$. The dashed line denotes our effective selection limit at $V=20.9$. These data have been transformed to the photometric scale of M98 to a precision of about 0.02 mag. 


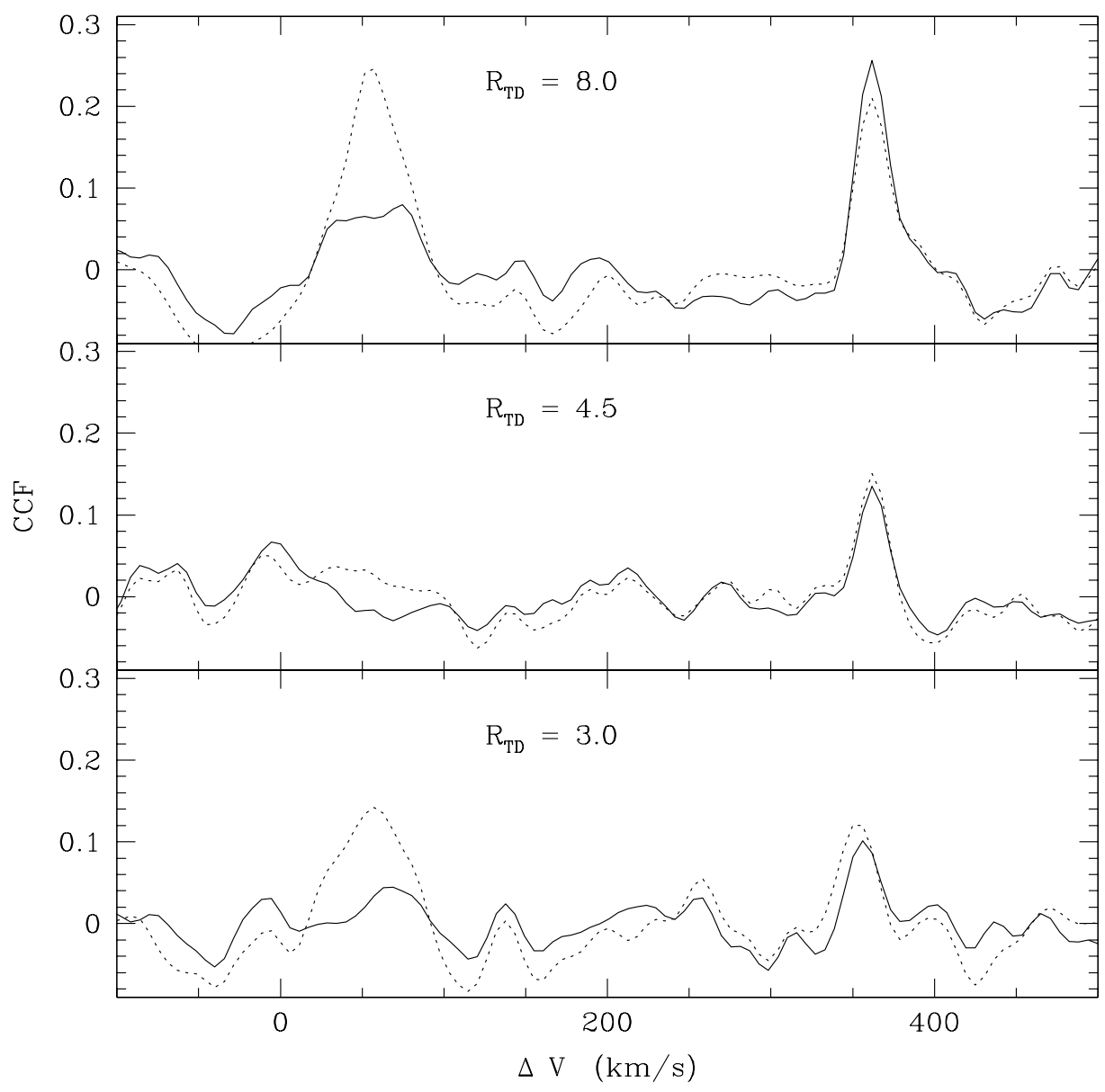

Fig. 3.- Raw Hectochelle cross-correlation functions from the IRAF task fxcor are plotted here in the velocity range -100 to $+500 \mathrm{~km} / \mathrm{s}$. Results for three Leo I stars observed during 2006 or 2007 are shown. The Tonry-Davis $R_{T D}$ (1979) values for these cases span much of the range exhibited by our kinematic sample (see Figure 4). Dotted lines correspond to crosscorrelations for spectra for which we did not do sky subtraction, while the solid lines are for sky-subtracted spectra. The broad, low-velocity peak in two of the non-sky-subtracted cases (top and bottom panels) is due to scattered moonlight (the middle panel is for a spectrum obtained in dark conditions). The velocity scale of these plots has not been corrected for the heliocentric velocity of the template. 


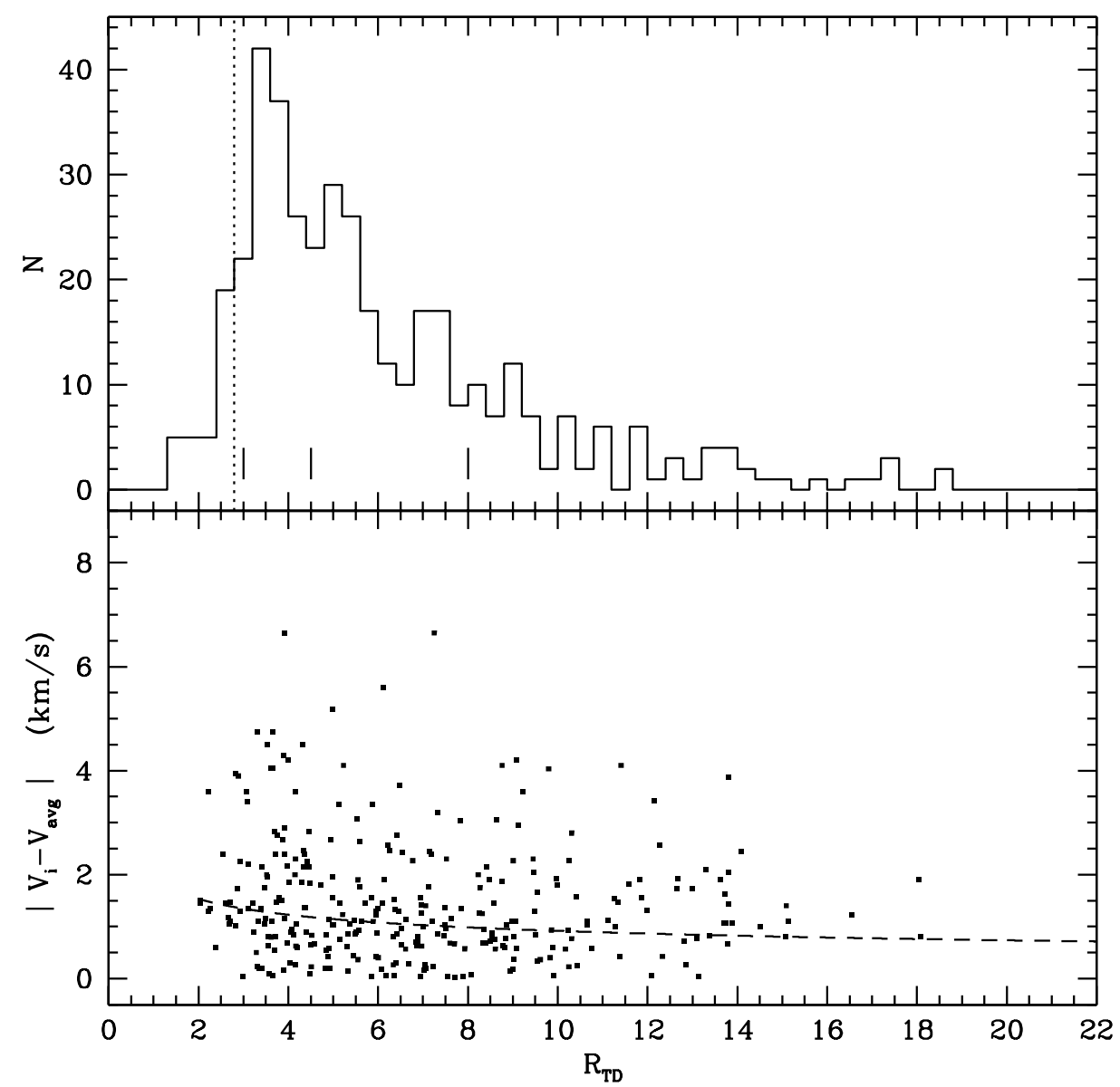

Fig. 4.- Top panel: The distribution of the Tonry-Davis $R_{T D}$ values for all stellar spectra in the Leo I sample (members and non-members included). The small tickmarks near the lower $\mathrm{x}$-axis indicate the $R_{T D}$ values of the three profiles shown in Figure 3. The median value of $R_{T D}$ is 4.9. Lower panel: A plot of the absolute value of the velocity differences of individual measurements $\left(V_{i}\right)$ and the weighted mean velocities $\left(V_{a v g}\right)$ for all stars in our sample with multiple velocity measurements. The dashed line is our error model, $\sigma_{i}=\sigma\left(R_{T D, i}: \alpha, x, \sigma_{0}\right)$ (see Section 3.2). The weights used to calculate $V_{a v g}$ are $w_{i}=1 / \sigma_{i, f x}^{2}$, where $\sigma_{i, f x}$ is the error estimate on $V_{i}$ from the IRAF cross-correlation routine fxcor. The weighted means for multiply-observed stars listed in Tables 4 and 5 are based on the values of $\sigma_{i}$ from the error model, so the means listed in the tables differ a bit from the values used here. 

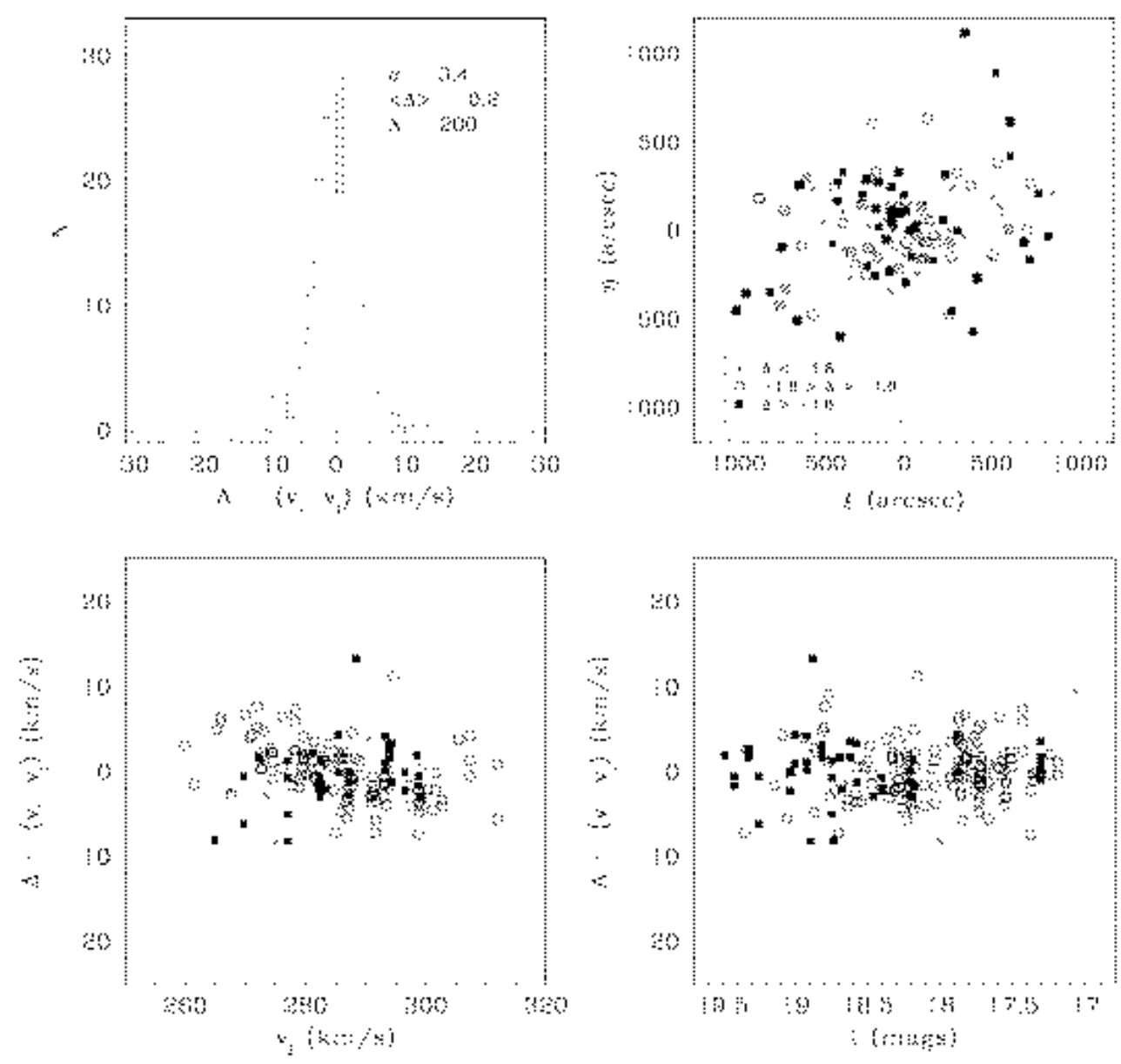

Fig. 5.- Upper left: The histogram of the velocity differences, $\Delta=v_{i}-v_{j}$ for stars with multiple velocity measurements. The indices $i$ and $j$ refer to the 2005, 2006 or 2007 Hectochelle data (see Table 1), with the chronologically earlier observation labeled with index $j$. The standard deviation, $\sigma$, mean offset, $\langle\Delta\rangle$, and total number of $\Delta$ values in the histogram are shown. Upper right: $\Delta$ as a function of standard coordinates, $\xi, \eta$, with different symbols denoting different ranges in $\Delta$ as noted in the legend. There is no evident trend of $\Delta$ with location on the sky apparent in this plot. Lower left: The distribution of $\Delta=v_{i}-v_{j}$ as a function of $v_{j}$. The crosses correspond to the case where $v_{j}=v_{2005}$; the open circles are for $v_{j}=v_{2006}$, and the filled squares are for $v_{j}=v_{2007}$. This panel reveals no statistically significant dependence of $\Delta$ on fiber configuration or run. Lower right: The distribution of $\Delta$ and $I$ magnitude. The symbols are the same as in the lower left plot. We find no dependence of $\Delta$ with $I$ magnitude. 

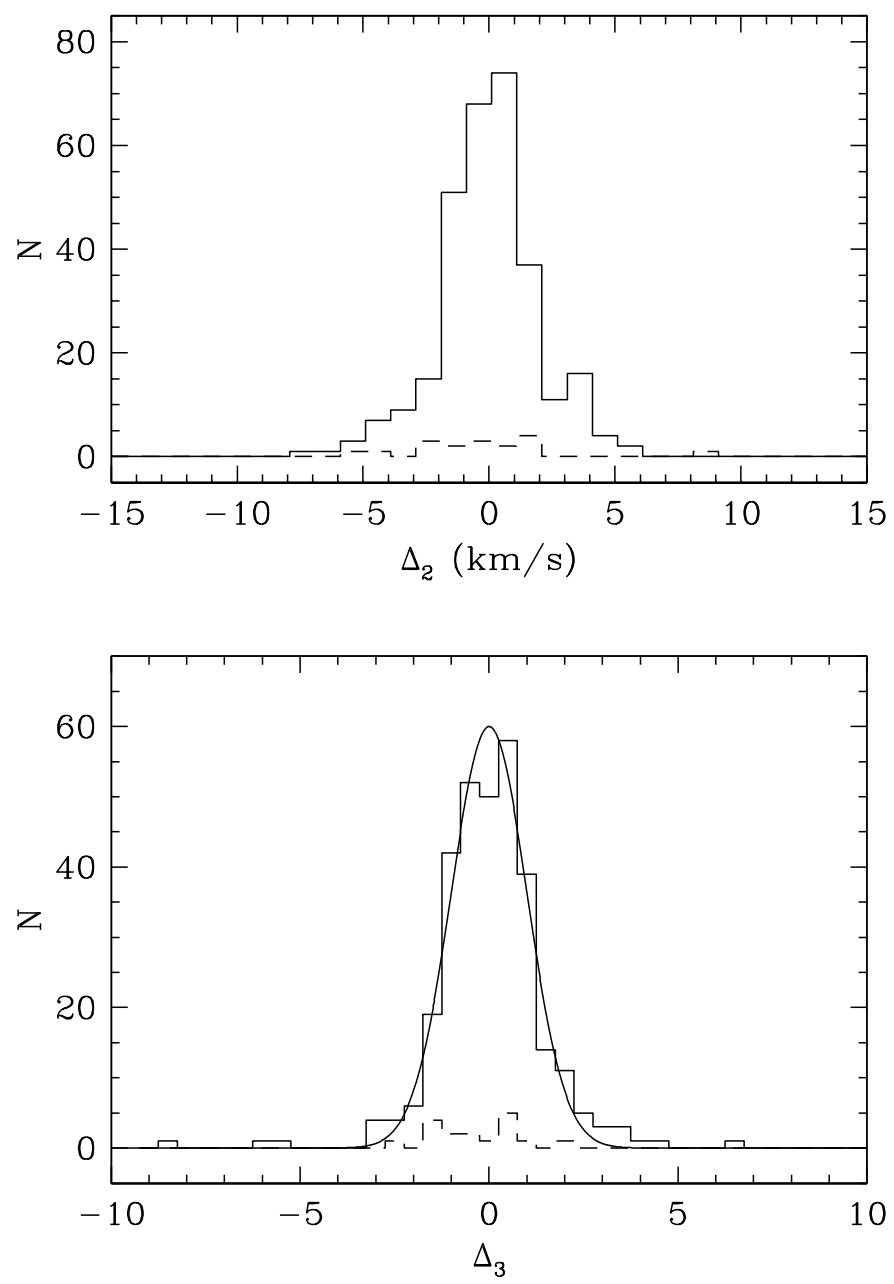

Fig. 6.- Upper plot: Histogram of velocity differences relative to the mean velocity, $\Delta_{2} \equiv$ $v_{i}-\langle v\rangle$ (not the same $\Delta$ plotted in Figure 5), for all stars with repeat measurements within the MMT dataset only (solid line) and for stars with both Keck and MMT velocity measures (dashed line). Lower plot: The same as the upper plot except for the statistic $\Delta_{3} \equiv \Delta v /\langle\sigma\rangle$. The curve is a Gaussian with unit $\sigma$. 

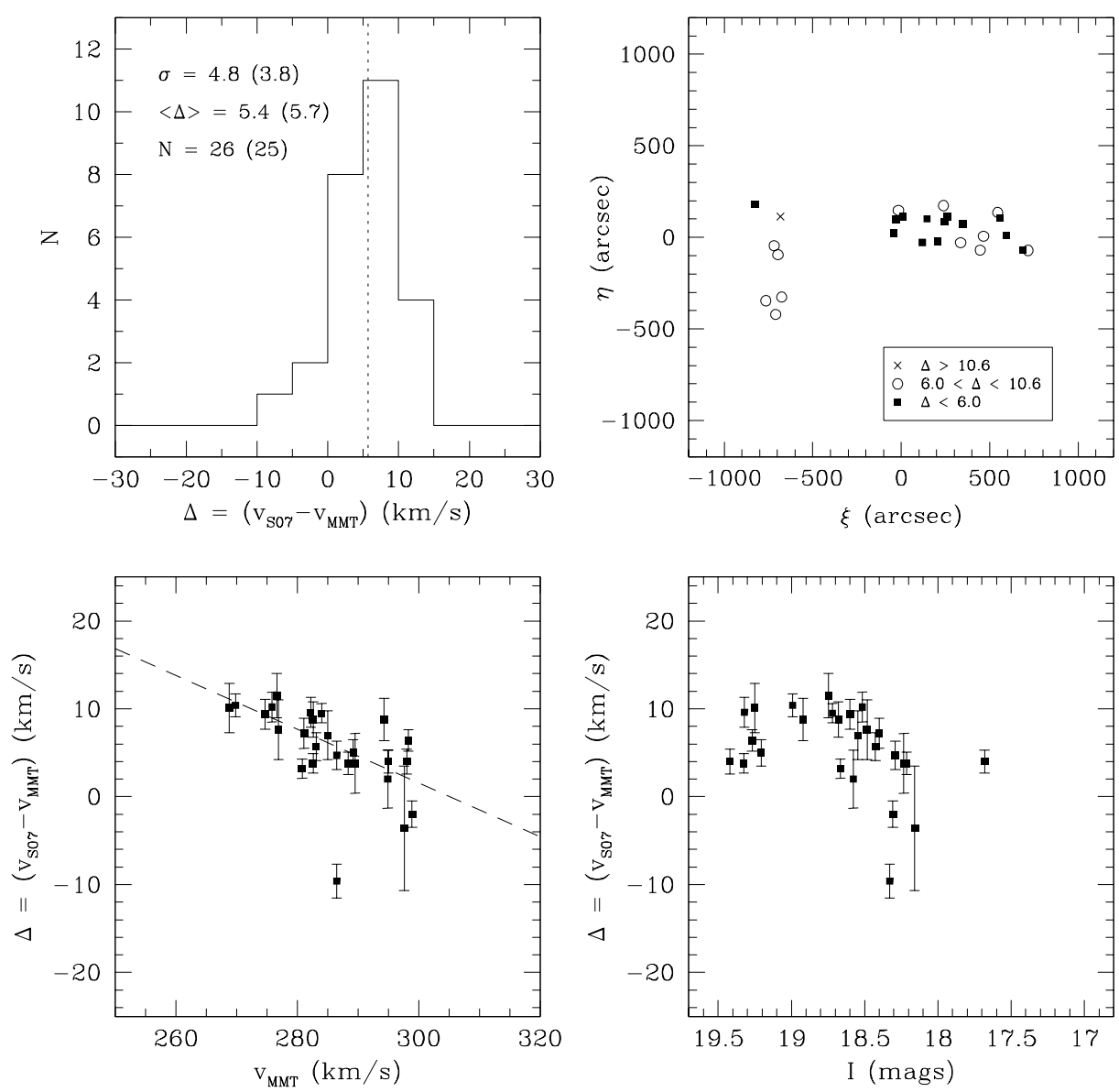

Fig. 7.- Upper left: A histogram of the velocity differences $\Delta=v_{S 07}-v_{M M T}$ where 'S07' refers to the results from Sohn et al. (2007). The standard deviation, $\sigma$, mean offset $\langle\Delta\rangle$, and number of $\Delta$ values are shown in the panel. Values in parentheses are for the sample that excludes the outlying point at $\Delta \sim-9.5$. Upper right: The dependence of $\Delta$ on standard coordinates, $\xi, \eta$. We find no significant trend of position with $\Delta$. Lower left: The distribution of $\Delta$ vs $v_{M M T}$. A strong trend is apparent. The line corresponds to a least-squares fit to the data (excluding the one outlier at $\Delta \sim-10$ ) of the form $\Delta=$ $-0.31(0.06) v_{M M T}+93.4(18)$ (1- $\sigma$ parameter errors are in parentheses). The correlation coefficient for this fit is $|R|=0.72$ for $N=25$, corresponding to a probability of $0.02 \%$ of obtaining a better linear fit by chance. Lower right: A plot of $\Delta$ vs. I-band magnitude. A least-squares linear fit suggests that there is no significant correlation between $\Delta$ and $I$. 

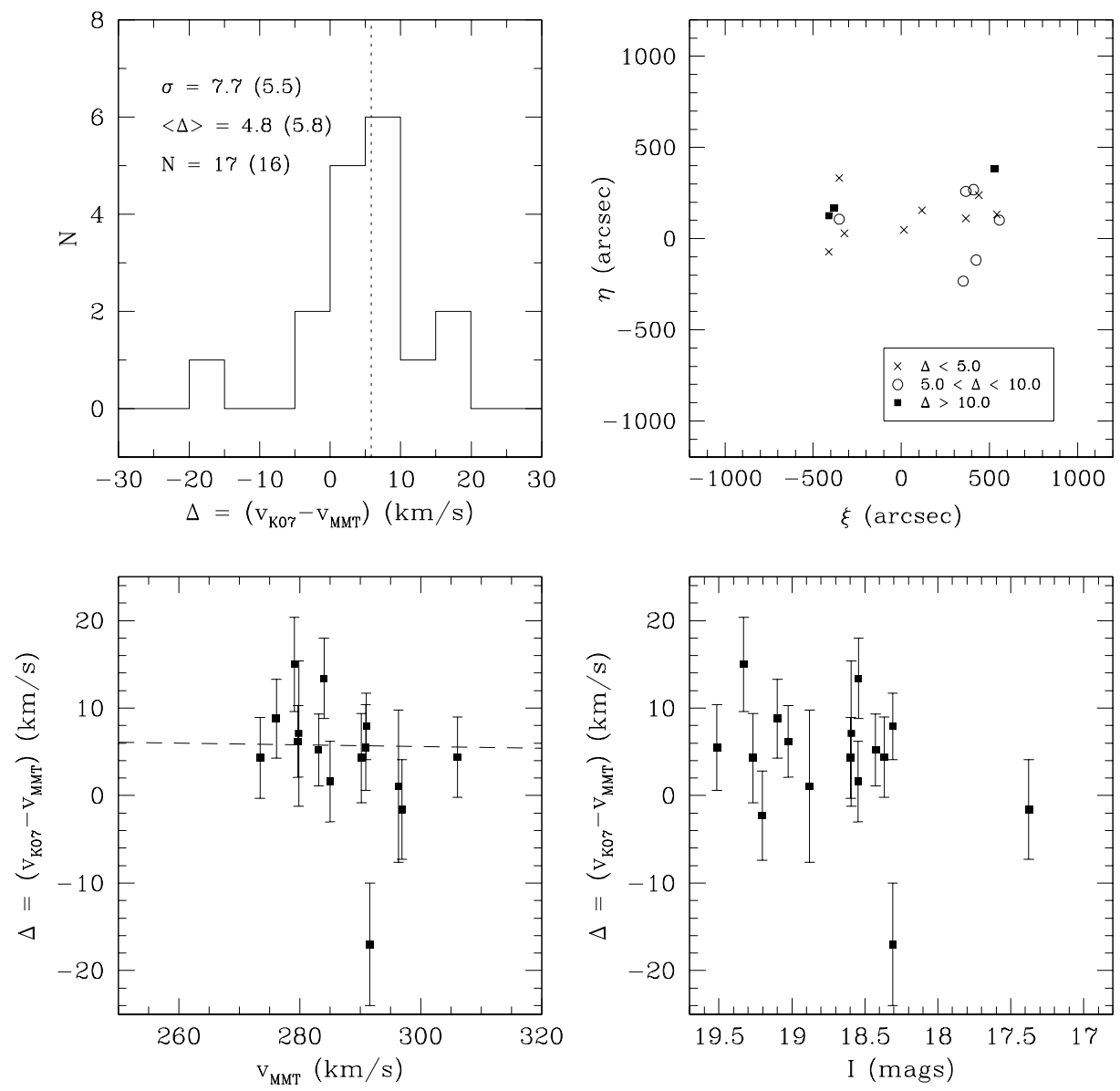

Fig. 8.- Upper left: A histogram of the velocity differences $\Delta=v_{K 07}-v_{M M T}$ where 'K07' refers to the results from Koch et al. (2007). The standard deviation, $\sigma$, mean offset $\langle\Delta\rangle$ (dotted line), and number of $\Delta$ values are plotted in the panel. Values in parentheses exclude the outlier at $\Delta \sim-18$. Upper right: The dependence of $\Delta$ on standard coordinates, $\xi, \eta$. We find no significant trend of position with $\Delta$. Lower left: The distribution of $\Delta$ vs $V_{M M T}$. The dashed line is a least-squares linear fit to the data (excluding the one outlier at $\Delta \sim-17$ but is consistent with $\Delta=$ constant $=4.8 \mathrm{~km} / \mathrm{s}$. Lower right: A plot of $\Delta$ vs. I-band magnitude. Apart from the mean offset, we see no significant trend in this plot. 


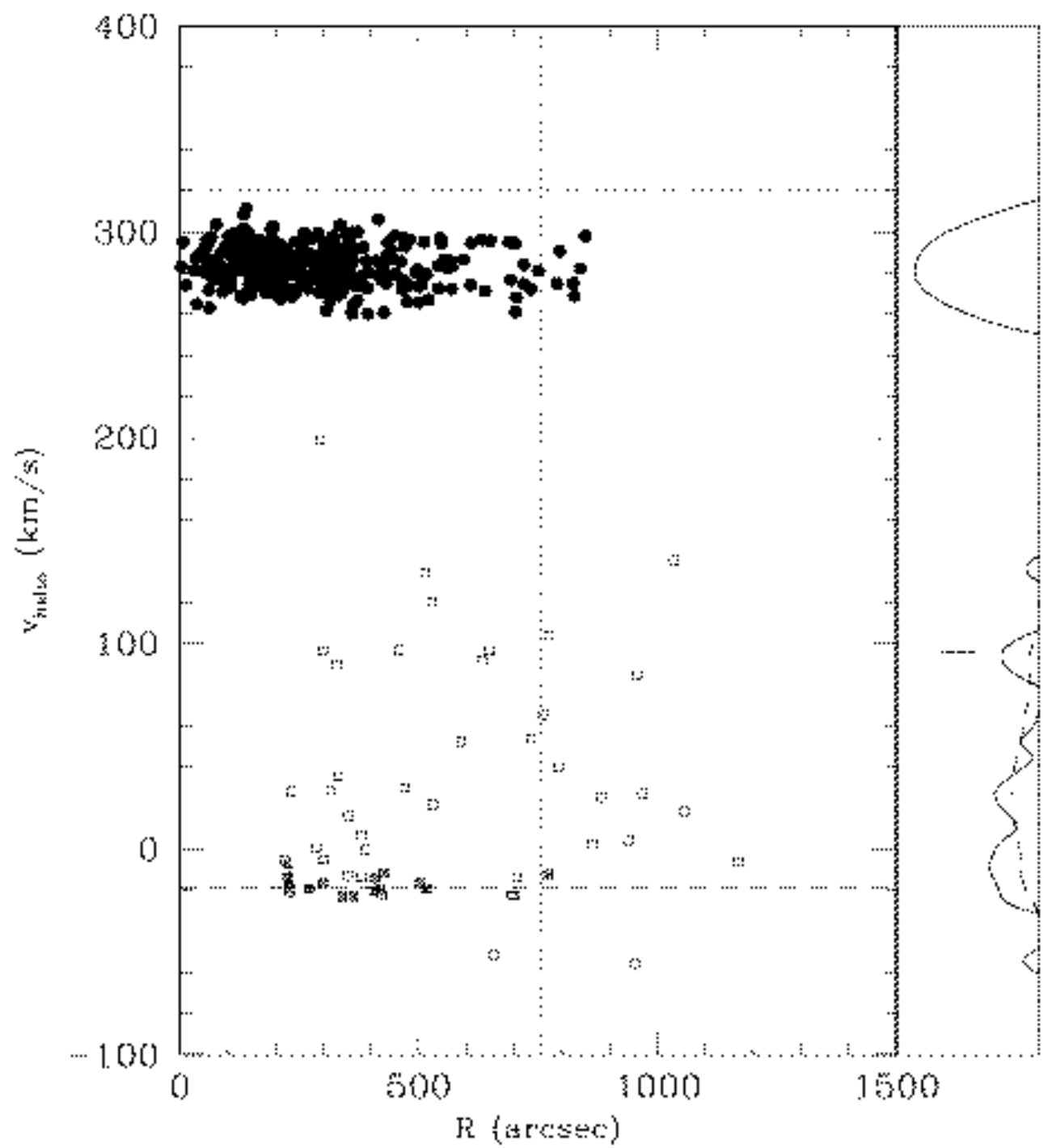

Fig. 9. - The radial distribution of heliocentric radial velocities in Leo I for all targets with $R_{t d} \geq 2.8$ (Table 5). The filled circles represent stars we take to be Leo I kinematic members. The dotted lines illustrate a crude Leo I selection region between $v_{\text {helio }}=250$ and $320 \mathrm{~km} / \mathrm{s}$, but it is clear that the identification of highly probable Leo I members is not at all ambiguous in this sample. The open squares denote likely non-members. The dashed horizontal line shows the typical heliocentric offset applied to the spectra; spectra from which we measured reflected sun/moonlight would have velocities near this line. The open squares with crosses denoting 'false positives', cases where we have likely measured the sky velocity $\left(-29 \leq v_{\text {helio }} \leq-11 \mathrm{~km} / \mathrm{s}\right.$ and $\left.R_{t d} \leq 4.2\right)$ rather than the velocity of an actual star in nonsky fibers (see Section 3.2; Table 5). The vertical dotted line marks the King tidal radius of Leo I from IH95 (756 arcsec; Table 6). At the right is the logarithmic histogram of the velocity distribution where each star denoted as a solid symbol is represented by a Gaussian of unit area with $\sigma=3.0 \mathrm{~km} / \mathrm{s}$. The dashed curve in the histogram is the predicted field star

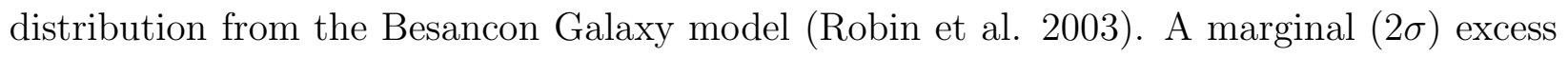
of stars relative to the model at $v_{\text {helio }} \sim 96 \mathrm{~km} / \mathrm{s}$ is marked (see Section 4.5 ). 


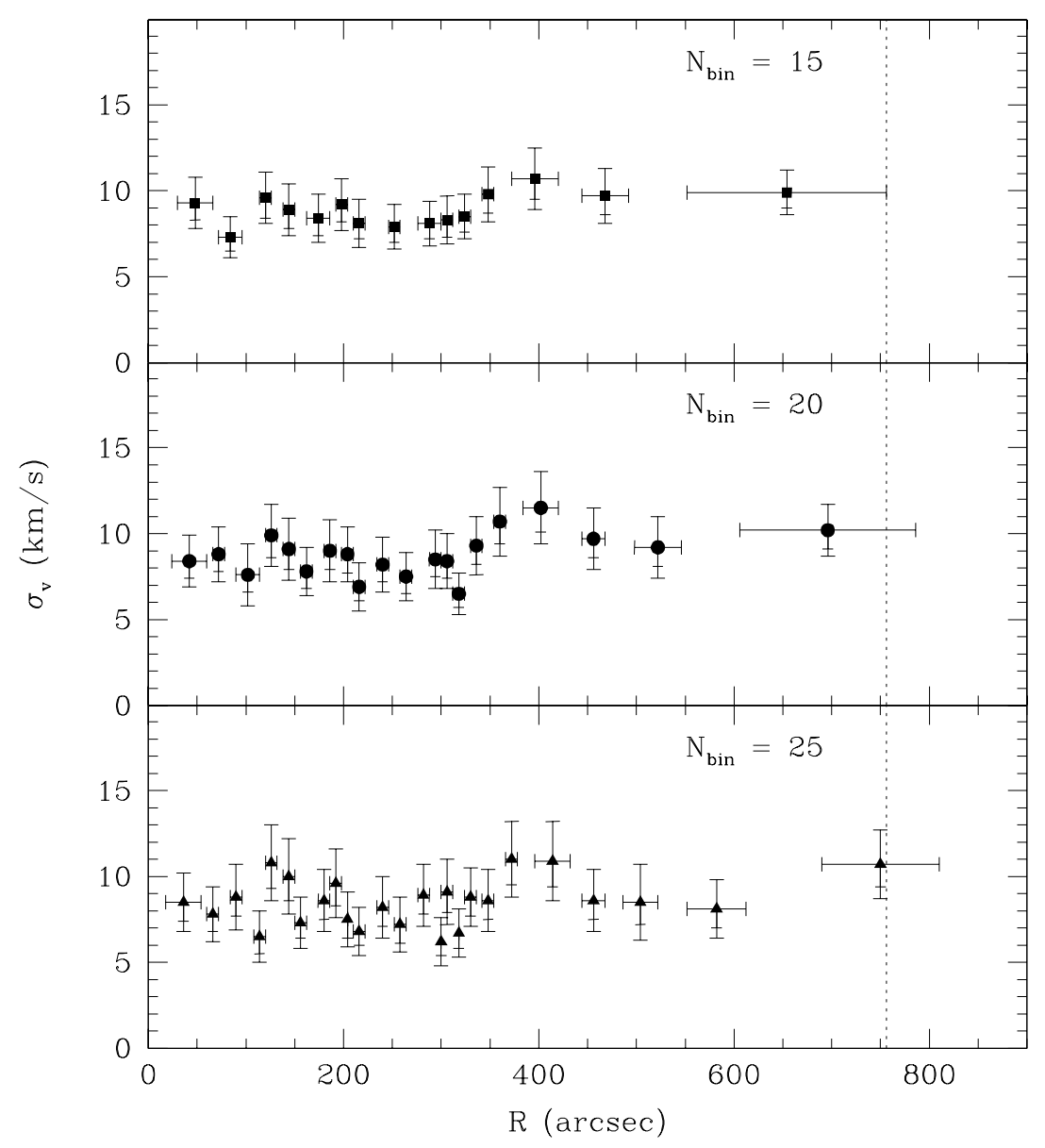

Fig. 10. - The Leo I velocity dispersion profile for all 328 members for three different binnings. For a given profile, the bins have nearly equal numbers of stars approximately equal to $328 / N_{\text {bin }}$. The radius, $R$, of each bin is the mean radius for all stars in that bin. The horizontal 'error bars' in $R$ is the standard deviation of the radius of the stars in each bin. The dispersion values, $\sigma_{v}$, have two downward error bars. The smaller errors are based on the method described by Kleyna et al. (2004), while larger errorbars correspond to the uncertainties calculated using the method described by Walker et al. (2006a, 2007a) and are equal to the upward error bars. The vertical dotted line shows the location of the 'tidal' radius of Leo I (756 arcsec) from IH95. 


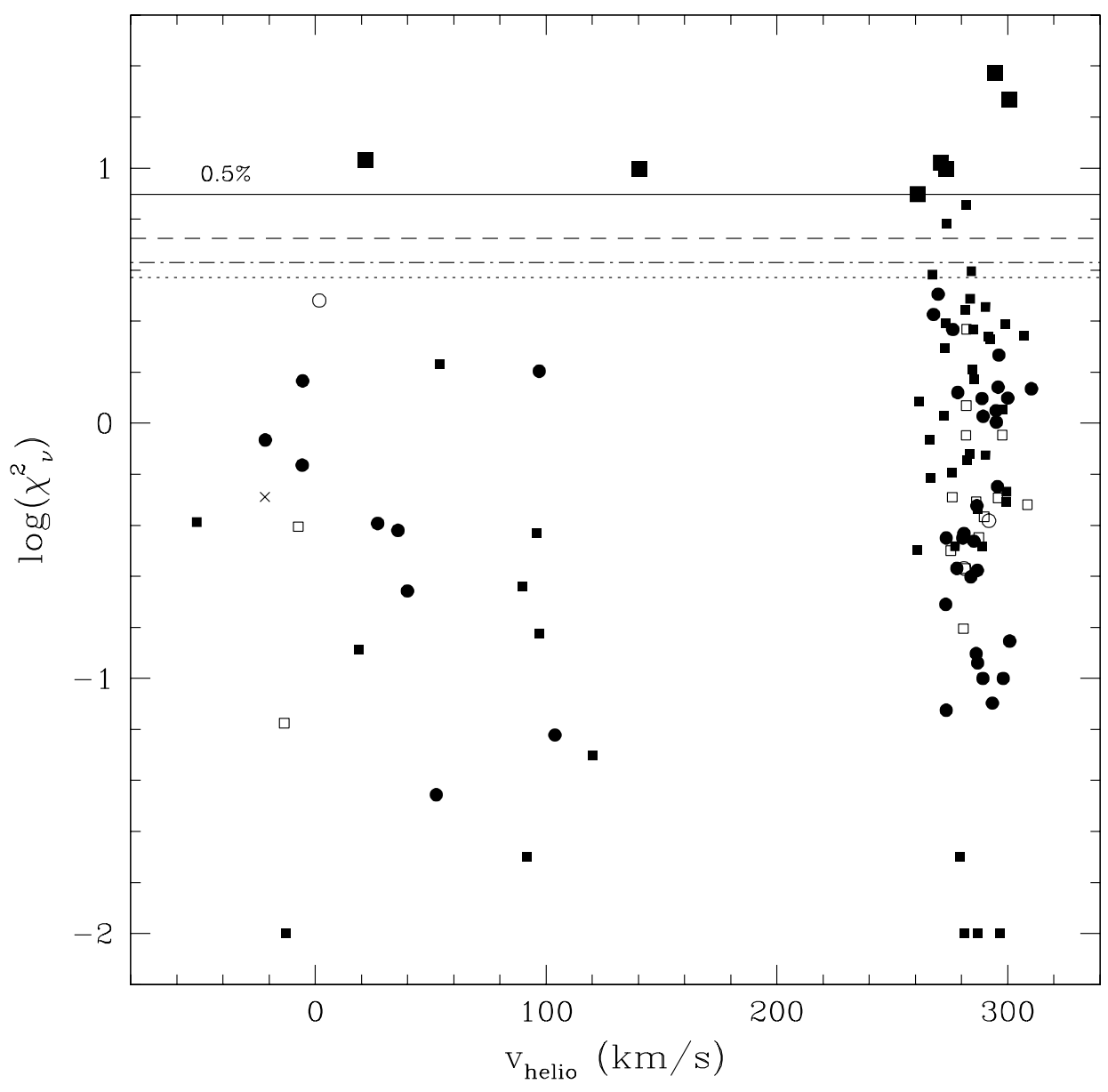

Fig. 11.- Distribution of the reduced chi-squared statistic, $\chi_{\nu}^{2}$, as a function of heliocentric velocity, $v_{h}$. The symbols denote the number of observations per star: $N=2$, filled squares; $N=3$, filled circles; $N=4$, open squares; $N=5$, open circles; $N=6$, cross. The lines denote the values of $\chi_{\nu}^{2}$ for which there is a $0.5 \%$ chance to exceed $\chi_{\nu}^{2}$ by chance in a normal distribution for different degrees of freedom, $\nu=N-1$. From top to bottom, the lines correspond to $\nu=1$ (solid line), 2 (dashed line), 3 (dot-dash line) and 4 (dotted line). The $\nu=5$ line is far above the sole 6 -observation point and is not shown. The large symbols denote the seven objects with $p\left(>\chi_{\nu}^{2}\right)<0.005$. These seven stars are noted in Table 5 . 


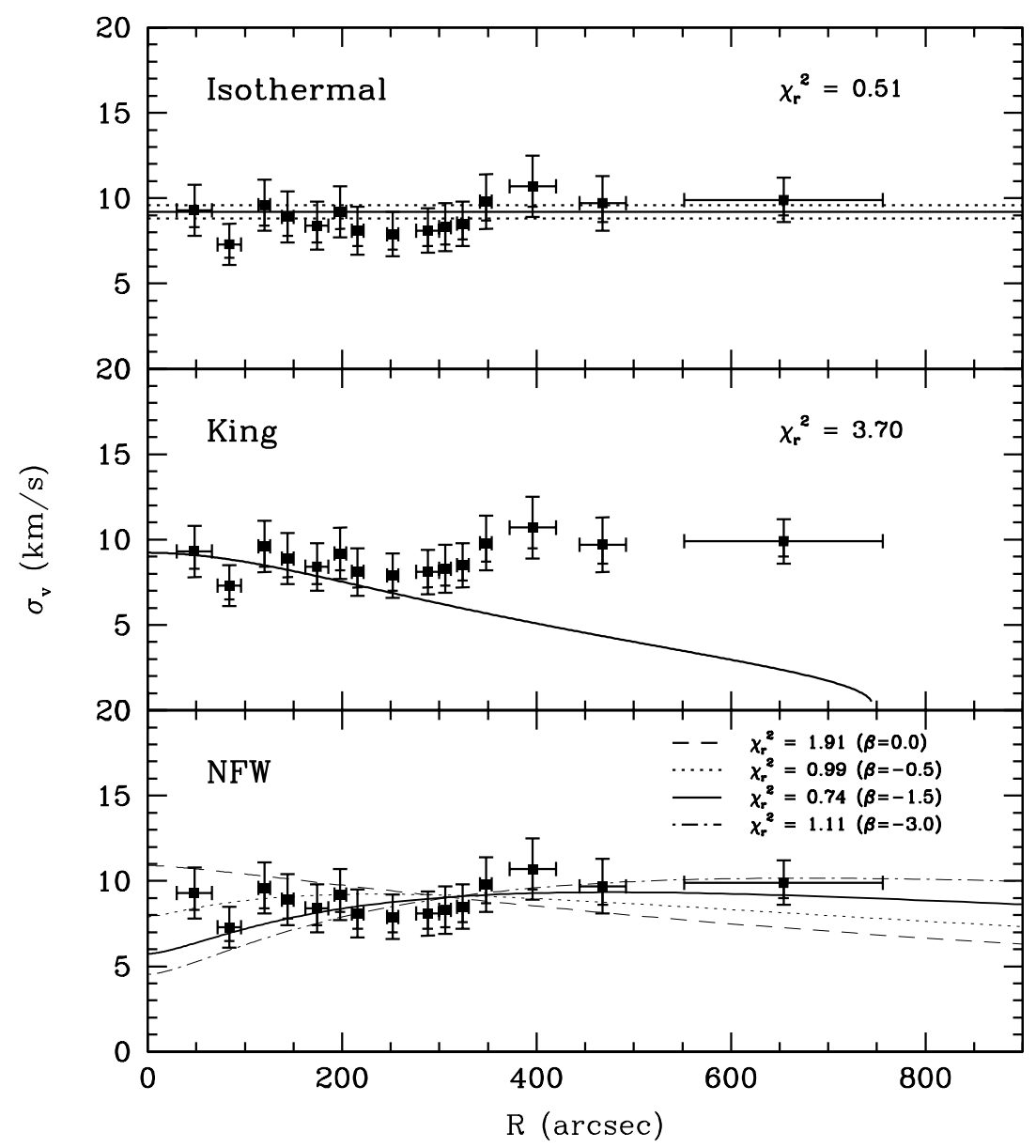

Fig. 12.- Comparisons of the binned radial velocity dispersion profile for Leo I ( $N_{b i n}=15$; see Figure 10) with equilibrium dynamical models. Top panel: The dispersion profile for an isothermal sphere set to have the dispersion equal to that of the entire sample (solid line; $\sigma=9.2 \pm 0.4 \mathrm{~km} / \mathrm{s}$ ). The uncertainty in the mean dispersion is denoted by the dotted lines. Middle panel: The dispersion profile for a King model with a central dispersion equal to the sample mean, and a concentration parameter of $c=0.58$ (IH95). Bottom panel: Dispersion profiles for NFW models assuming an isotropic velocity distribution ( $\beta=0$; solid line) and two tangentially anisotropic distributions $(\beta=-0.5$, dashed line, and $\beta=-1.0$, dotted line). All of these fits include a visible and dark component, corresponding to a total mass of $9 \times 10^{8} M_{\odot}$. Further model details are given in Section 4.2. Values of reduced $\chi_{r}^{2}$ are shown for each model 'fit' in each panel. 


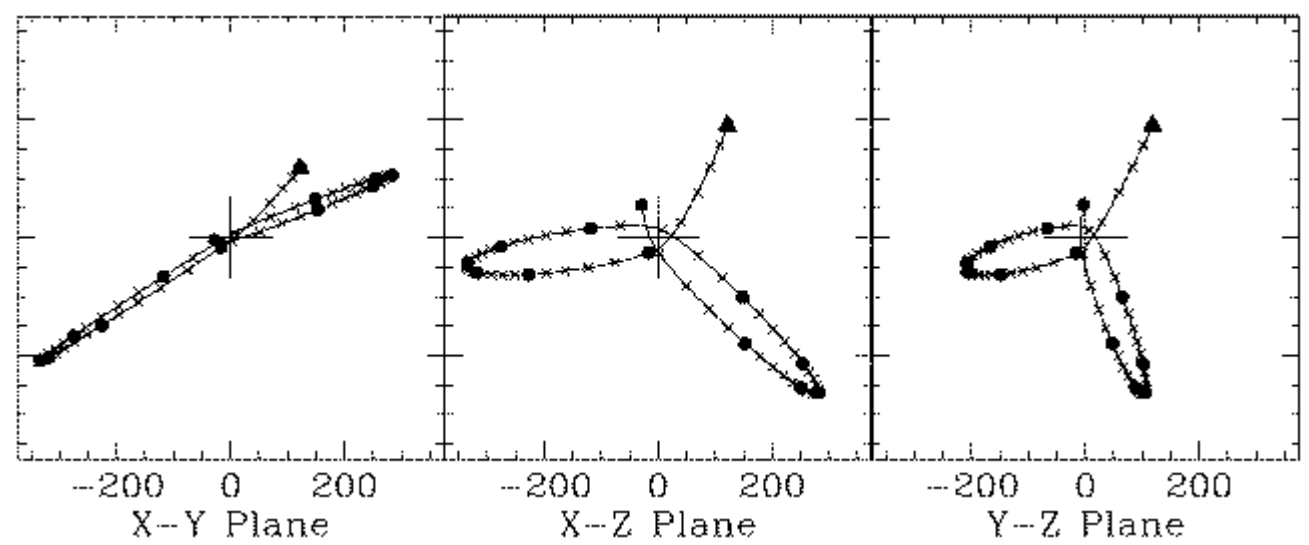

Fig. 13.- Orthogonal projections of a plausible Leo I orbit that has $R_{\text {peri }}=15 \mathrm{kpc}$ and the preferred orbital pole of $\mathrm{S} 07[(l, b)=(122,13)]$ (similar to the orbits detailed in Table 8). Crosses are separated by 200 million years along the orbit, while filled circles represent locations along the orbit every 1 Gyr. The large triangle denotes the present location of Leo I. The orbit is shown for the past 12 Gyr in an assumed static Galactic potential. The large cross in each panel denotes the Galactic Center. These orbits were calculated using the static, multi-component potential of Johnston et al. (1995) to represent the Milky Way, and a halo with an asymptotic rotation velocity of $190 \mathrm{~km} / \mathrm{s}$. 


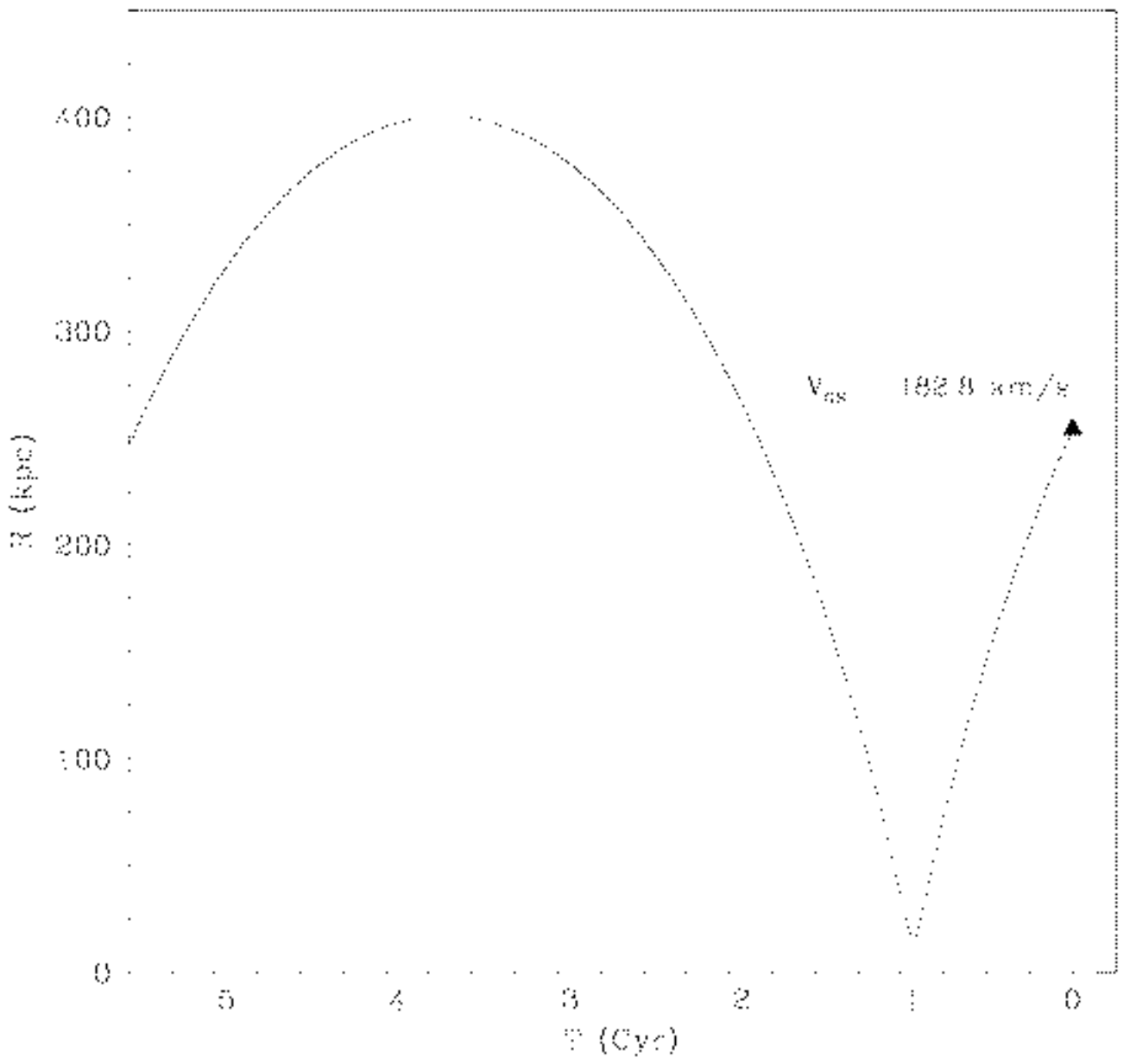

Fig. 14. - The Galactocentric distance of Leo I as a function of time (with $T=0$ corresponding to the present) for the orbit described in Figure 13. The triangle shows the (assumed) current location of Leo I. The galaxy's adopted Galactostationary radial velocity is noted. Only one orbital period, about $5.5 \mathrm{Gyr}$, is shown. The assumed galactostionary velocity used for this calculation differs somewhat from the value we observe for Leo I $\left(v_{G S}=174.9\right.$, but this has no significant impact on our analysis. 


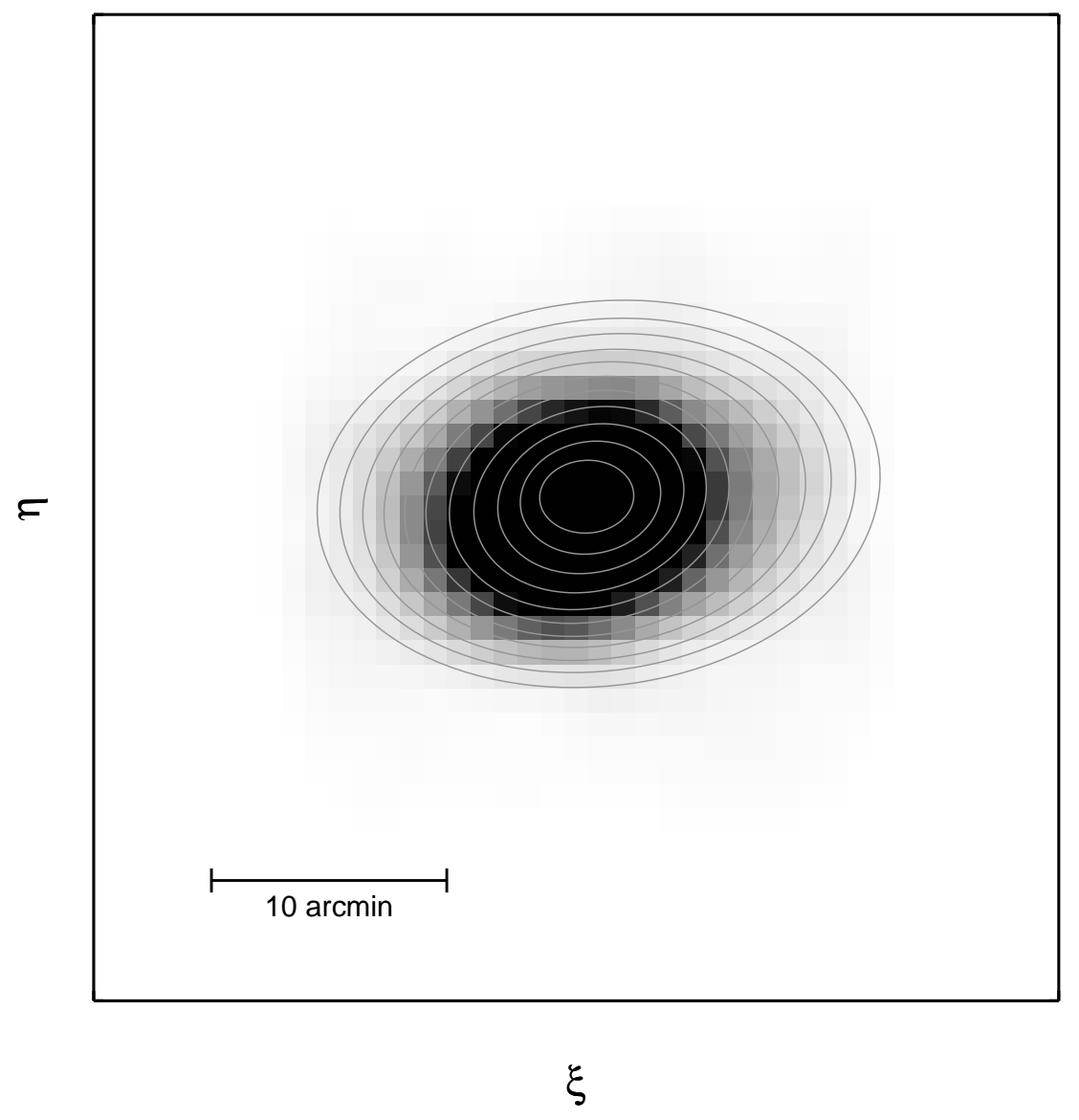

Fig. 15.- A greyscale image of the smoothed star counts of the 12630 objects identified in the selection region defined in Figure 2 and smoothed with a Guassian spatial filter with $\sigma=2$ arcmin. Superimposed are fitted ellipses to the isopleths. The ellipses have major axes ranging from 2 arcmin to 12 arcmin in 1 arcmin steps. This plot is oriented with North to the top, and East to the right. 


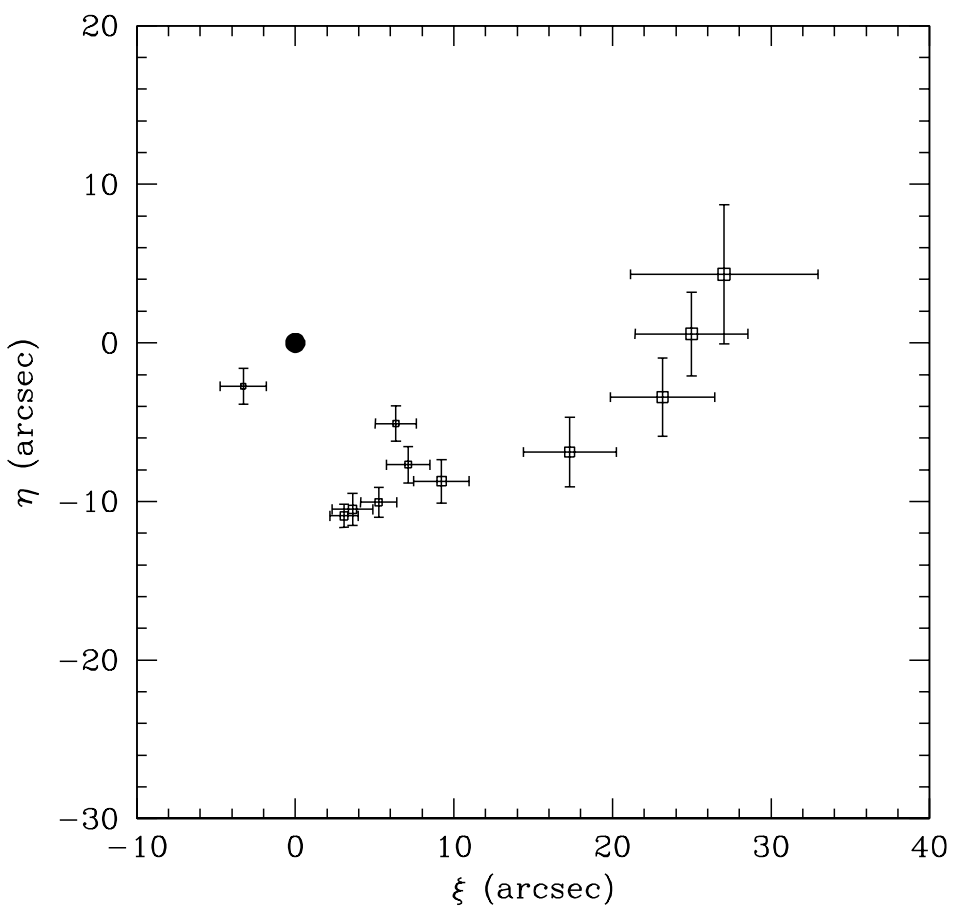

Fig. 16. - The centers of the fitted ellipses shown in Figure 15, The increasing symbol sizes denote increasingly larger ellipses in Figure 15, Note that the coordinates of the center of the fitted ellipses remain close to the adopted center location of Leo I (see Figure 11) for the smaller ellipses corresponding to the inner isopleths, but start to deviate to the NE for the 4-5 ellipses for the outermost isopleths. The centroids of the innermost 2-3 ellipses may be systematically slightly affected by the presence of gaps in the CCDs apparent in Figure 1. 


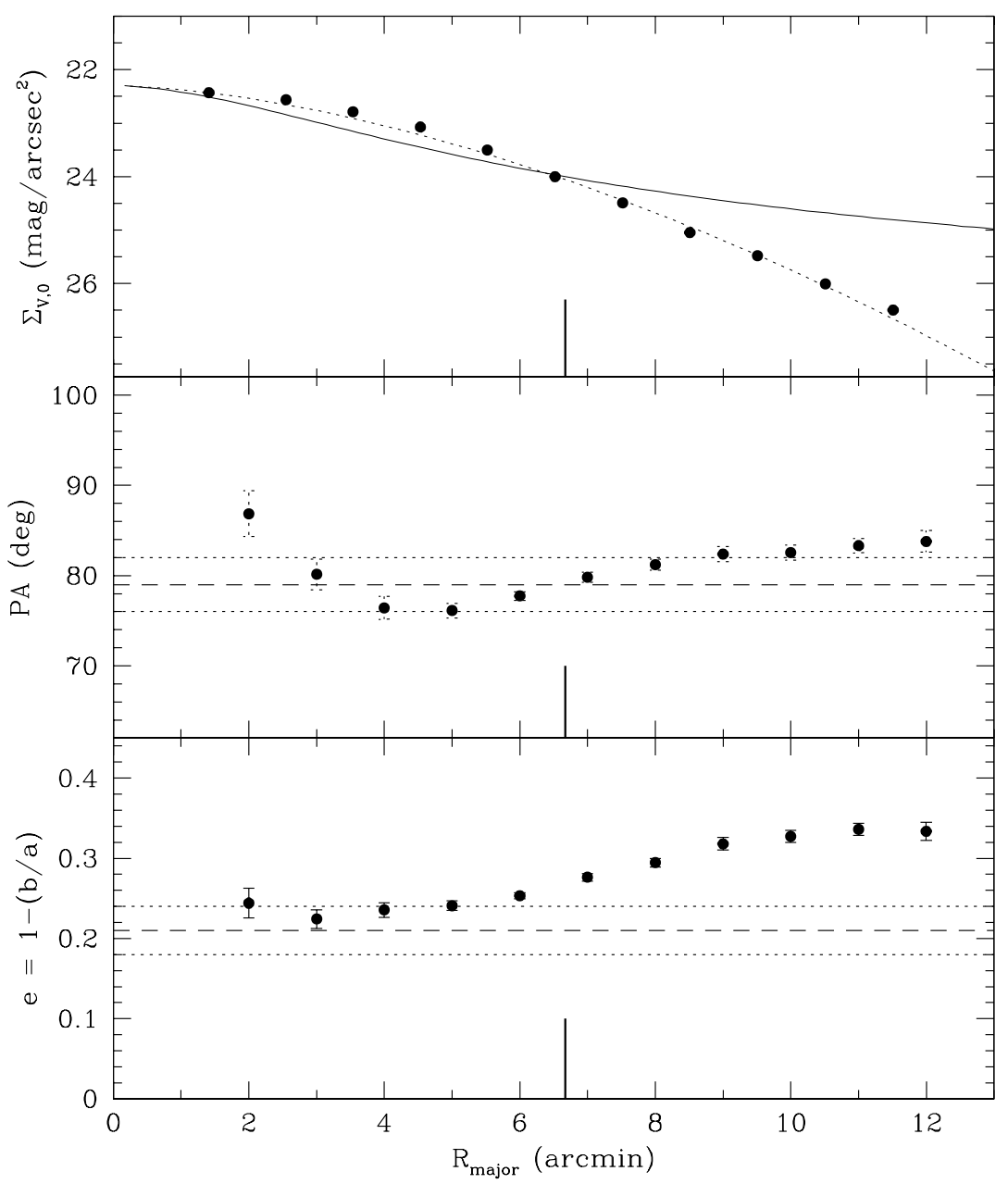

Fig. 17.- Top panel: The surface brightness profile for Leo I based on the isopleths in Figure 15 and measured in the elliptical annuli shown in that plot. The radii are for the geometric mean major axes for each annulus. This profile was produced assuming a constant baryonic $M / L$ ratio and the central surface brightness from IH95 (see Table 6 ). The solid line is the projected density distribution for an isothermal sphere with a core radius of 270 arcsec (equal to the King core radius; see Table 6). The dotted line is a Sersic profile with $m=0.6$ and a Sersic radius, $r_{S}$, of 300 arcsec. Middle panel: The position angle of the major axes of the fitted ellipses in Figure 15 as a function of major axis. Lower panel: The ellipticity of the fitted ellipses in Figure 15 as a function of major axis. The ellipticity is defined as $e=1-b / a$, where $a$ and $b$ are the major and minor axes, respectively. The horizontal lines in the lower two panels denote the position angle $(P A=79 \pm 3 \mathrm{deg})$ and ellipticity ( $e=0.21 \pm 0.02$ ) from IH95, consistent with the inner values of the profiles shown here. The short vertical lines in the panels are located at $R=400$ arcsec, the radius at which we claim to see a change in the kinematic properties of the stars in Leo I (Section 4.3.3). 


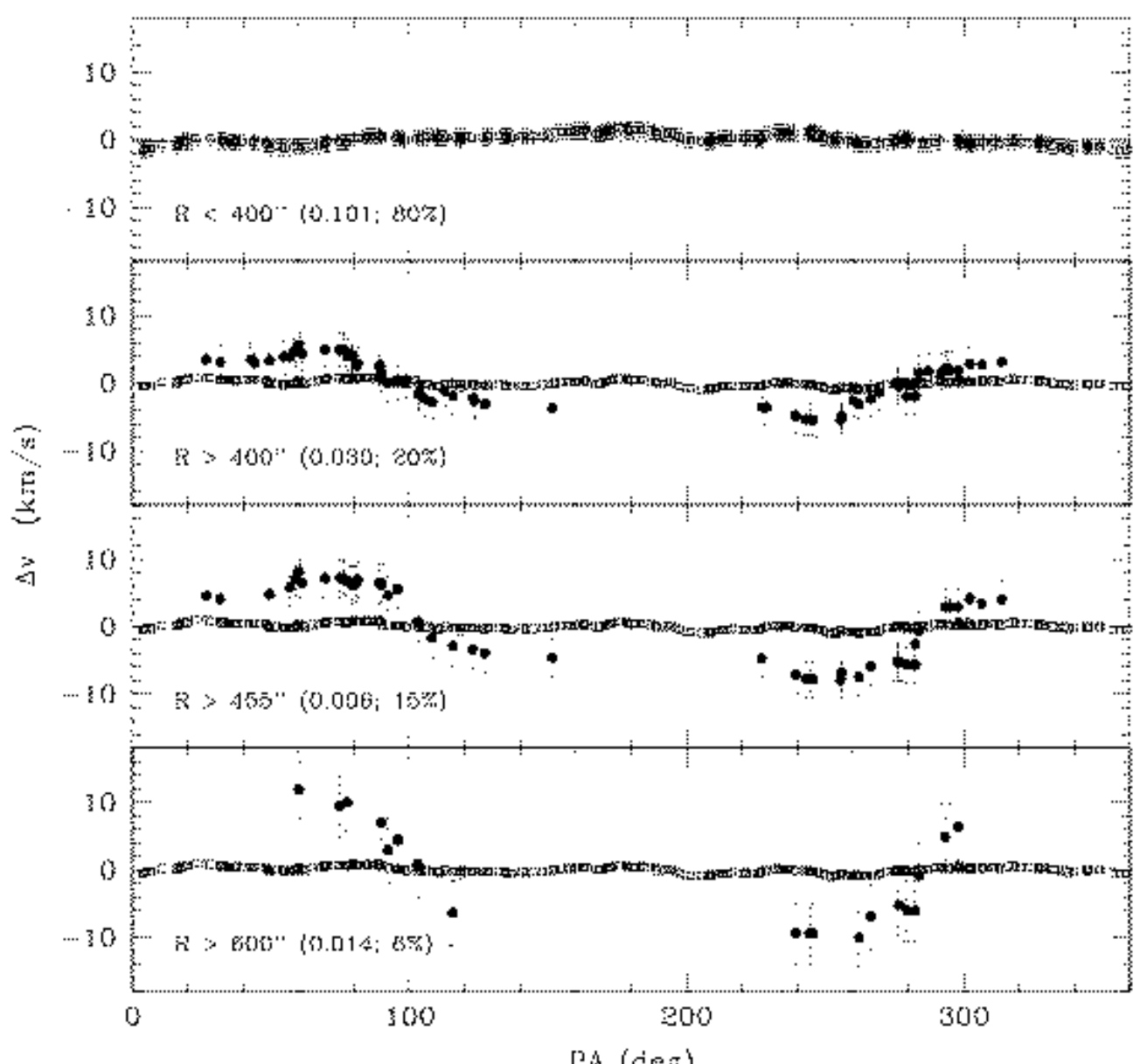

Fig. 18. - Plots of the bisector mean velocity difference, $\Delta v$, defined as the difference of the mean velocities of Leo I members on both sides of a bisector oriented along a given position angle, $P A$ (see Figure 22 and Section 4.3.3 for further details). For the data plotted with error bars, the radial range of the dataset (from top: $R<400$ arcsec, $R>400$ arcsec, $R>455$ arcsec, and $R>600$ arcsec), the probability of exceeding $(\Delta v)_{\max }$, and the fraction of all the Leo I members used to produce each plot are given in each panel. In the lower three panels the set of points (open squares) that lack error bars are for the full distribution of Leo I members $\left(N=328\right.$, and for which the probability of exceeding $(\Delta v)_{\max }$ is $\left.31.3 \%\right)$. The line at $\Delta v=0$ in the top panel is shown for reference. 


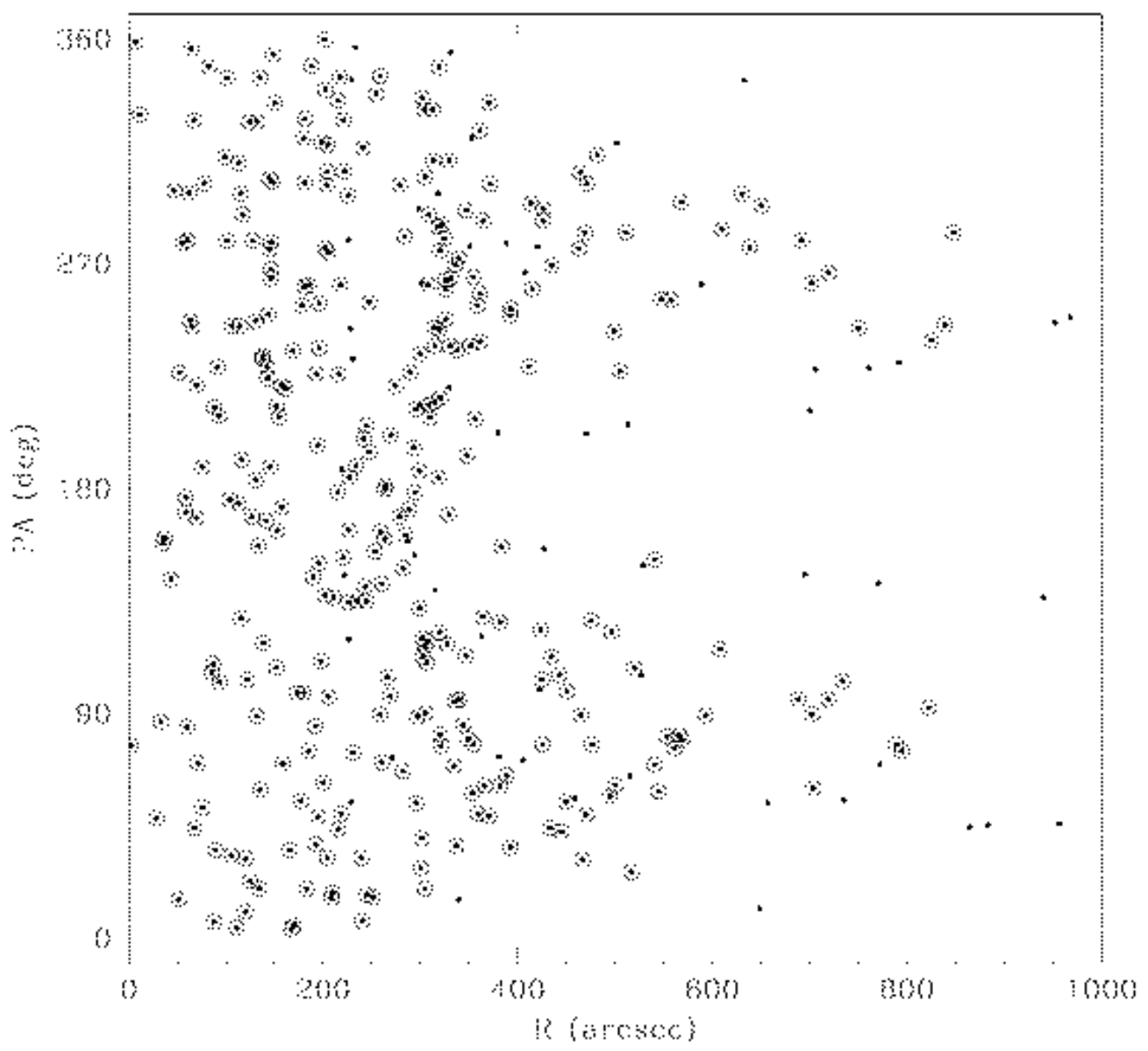

Fig. 19.- The position angle vs. radial distance from the center of Leo I for all stars listed in Table $5\left(R_{T D} \geq 2.8\right)$. The points enclosed in open circles are likely Leo I members. Note the transition from a fairly uniform distribution of members (in $P A$ ) for $R<400$ arcsec (denoted by the vertical dashed line) to a strongly bimodal distribution for $R>400$ arcsec. The non-members (small dots) show a noticeably more uniform distribution in PA regardless of $R$. 


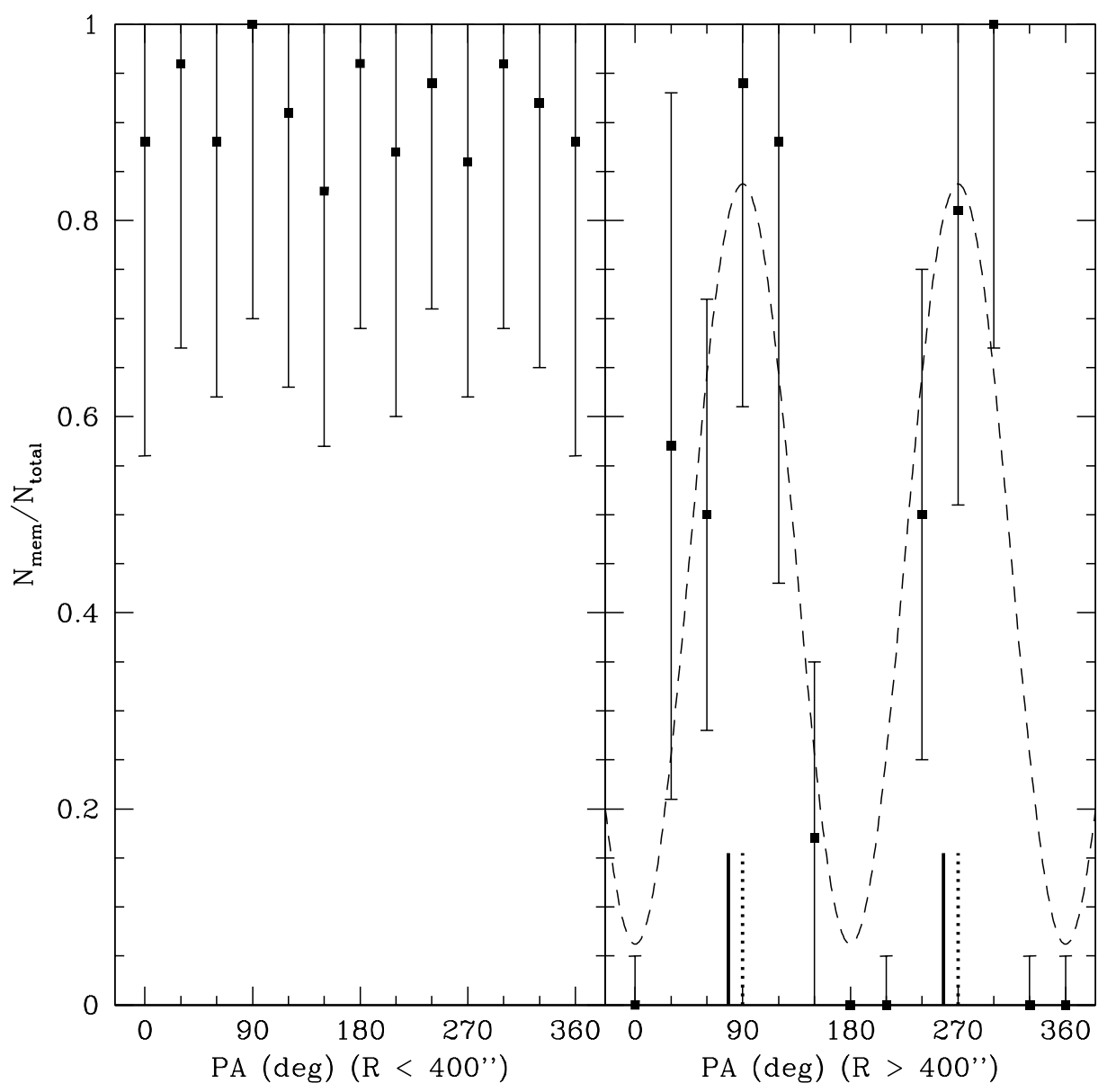

Fig. 20. - Plots of the fraction of members divided by the total stars observed kinematically (data from Table 5) as a function of position angle for stars in the inner sample $(R<400$ arcsec; left panel), and for stars of the outer sample ( $R \geq 400$ arcsec; right panel). The double sine curve is a fit to the outer sample data. The maxima occur at $P A=90$ and 270 degrees (short dotted vertical lines at the bottom of the plot). The major axis position angle of the inner regions of Leo I (see Figure 17) is denoted by the short vertical solid lines at $P A=78$ and 258 degrees. Bins in the right panel with values of $N_{m e m} / N_{\text {total }}=0$ and a small error bar contain no kinematic members but $N_{\text {total }} \geq 1$. The one bin with $N_{\text {mem }} / N_{\text {total }}=0$ and no error bar has $N_{\text {total }}=0$. 


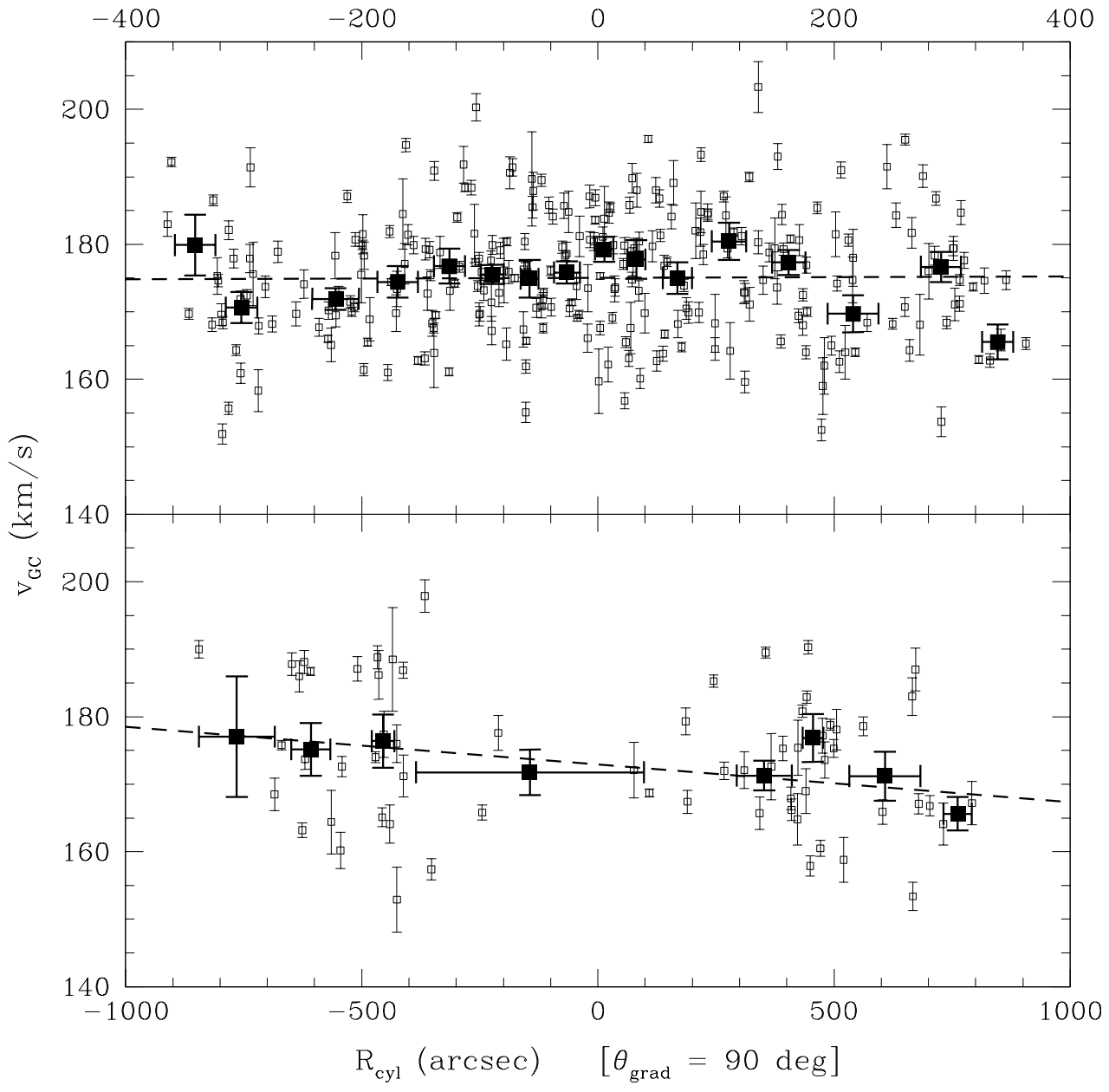

Fig. 21. - Top panel: A fit to the velocities corrected for Galactic rotation versus the $R_{c y l}$ relative to a minor axis with position angle $108 \mathrm{deg}$ (see text for details) for all stars with $R \leq 400$ arcsec (the inner sample). Lower panel: The same for stars with $R>400$ arcsec. In both panels the open squares are the individual velocities with their uncertainties. The larger filled squares are the binned mean velocities. For these larger symbols, the vertical error bars are the standard deviations of the mean velocity in each bin, while the horizontal error bars are the standard deviations of the radial coordinates of the sample of stars in each bin. The dashed lines show the least squared fit line to the respective binned mean velocities. Note the change in scale in the horizontal axes of the two panels. The slope of the fit in the lower panel is $-0.34 \pm 0.15 \mathrm{~km} / \mathrm{s} /$ arcmin, in the sense that the mean velocity becomes more negative toward $P A=90 \mathrm{deg}$. 


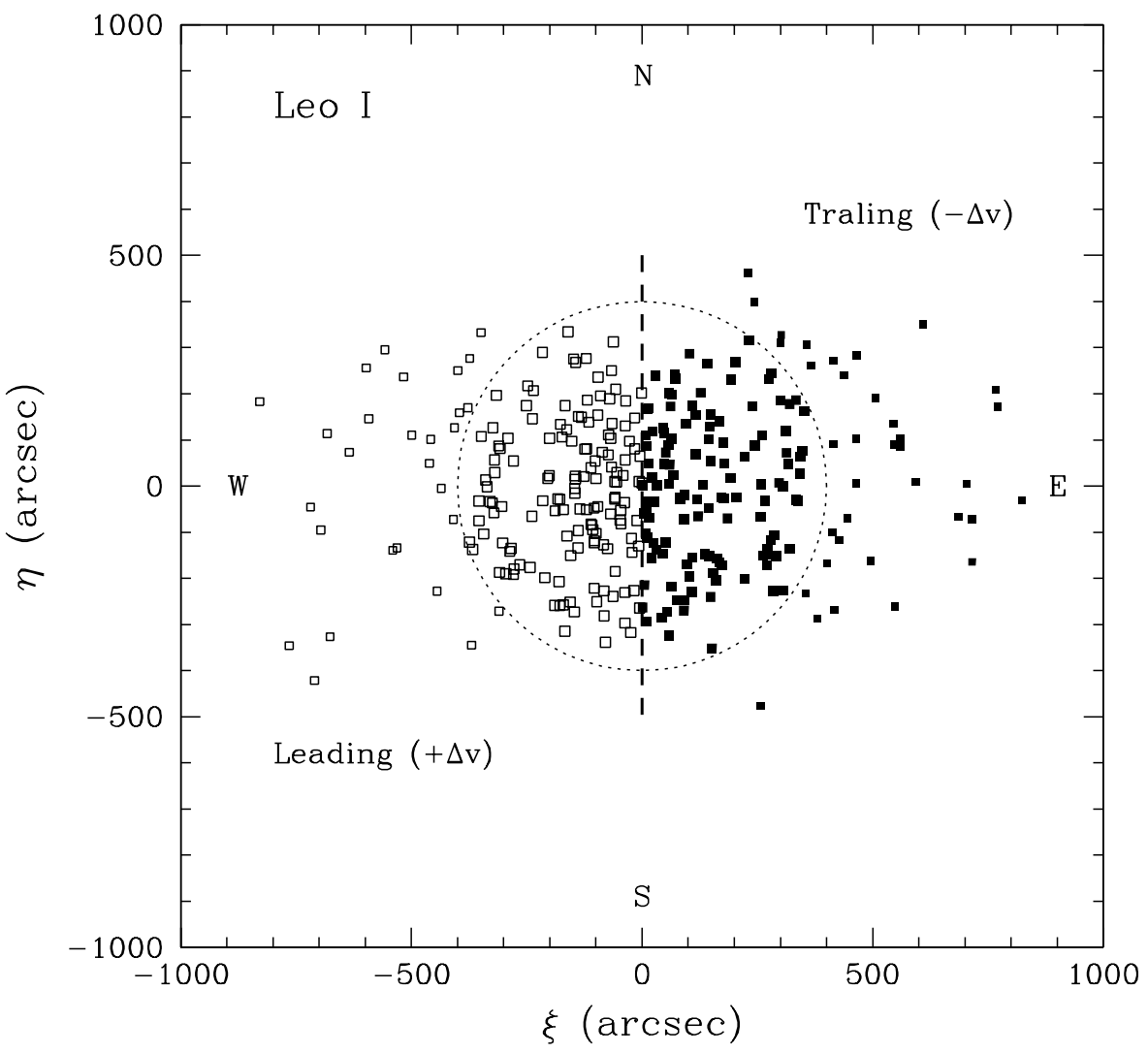

Fig. 22. - The spatial distribution of Leo I kinematic members. The dashed straight line indicates the position angle of an axis perpendicular to the maximum velocity gradient observed in Leo I. The circle (dotted line) corresponds to $R=400$ arcsec, the radius where we see a change in the internal kinematics in Leo I. This figure also illustrates how Figure 18 was constructed. The mean velocity of points on one side of the bisector (solid symbols) minus the mean velocity of points on the other side of the bisector (open symbols) represent the data that go into calculating $\Delta v$ for $P A=0$ degrees. This orientation corresponds to the maximum velocity gradient in Leo I along $P A=90 \mathrm{deg}$. Figure 18 plots how $\Delta v$ changes as the bisector is rotated clockwise in position angle. 


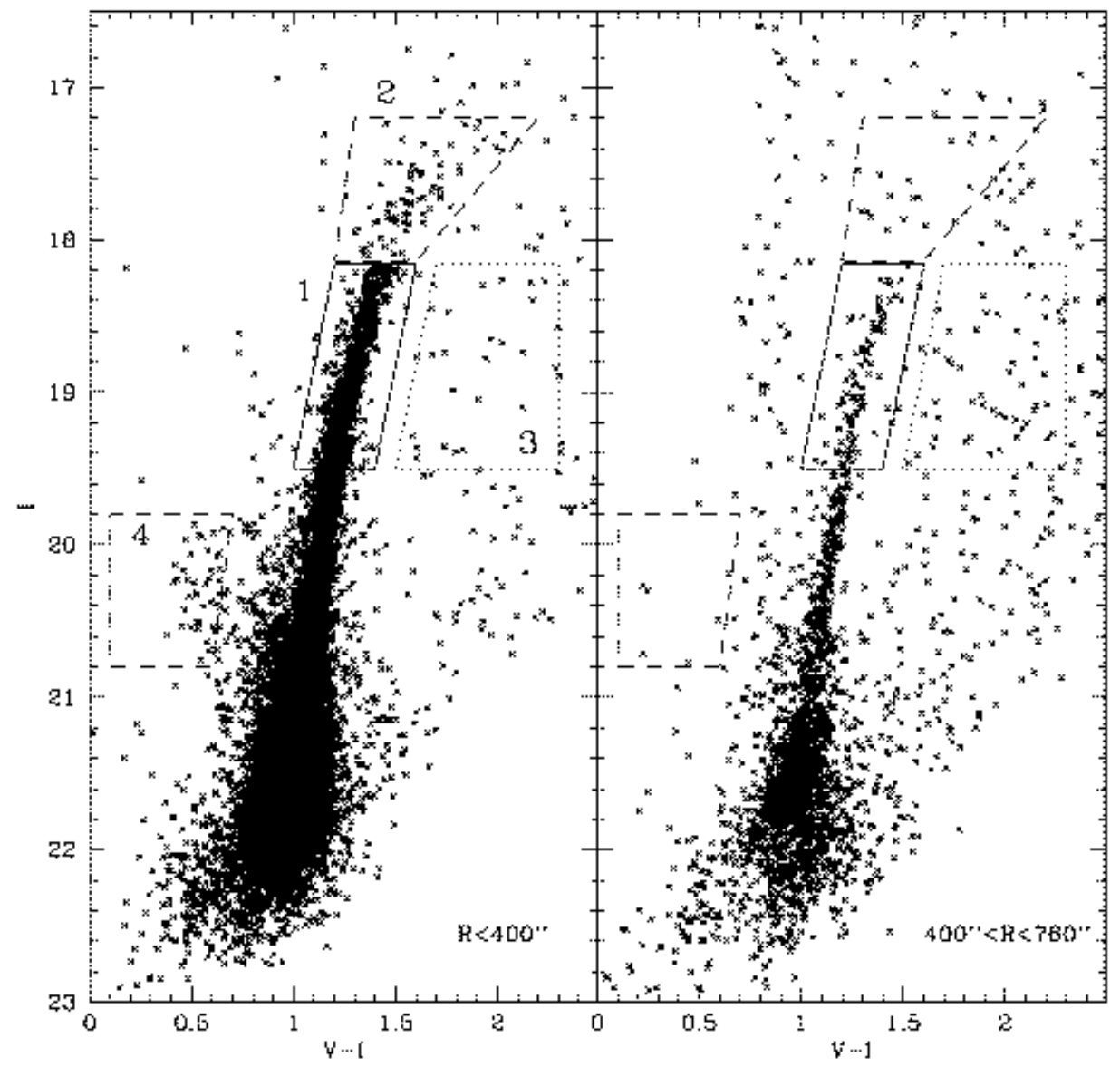

Fig. 23.- Left panel: The Leo I CMD for stars with $R_{b} \leq 400$ arcsec, where $R_{b}$ is the break radius' (Section 4.3.3). The four regions correspond to RGB stars (1; solid line), AGB stars (2; dashed line), field stars (3; dotted line) and blue-loop stars (4; dashed line). Right panel: The Leo I CMD for stars with $400<R \leq 760$ arcsec. The CMD regions are the same as in the left panel but the areal coverage in the right panel is 3.2 times that of the left panel. Star counts for the regions defined in these CMDs are listed in Table 7. 


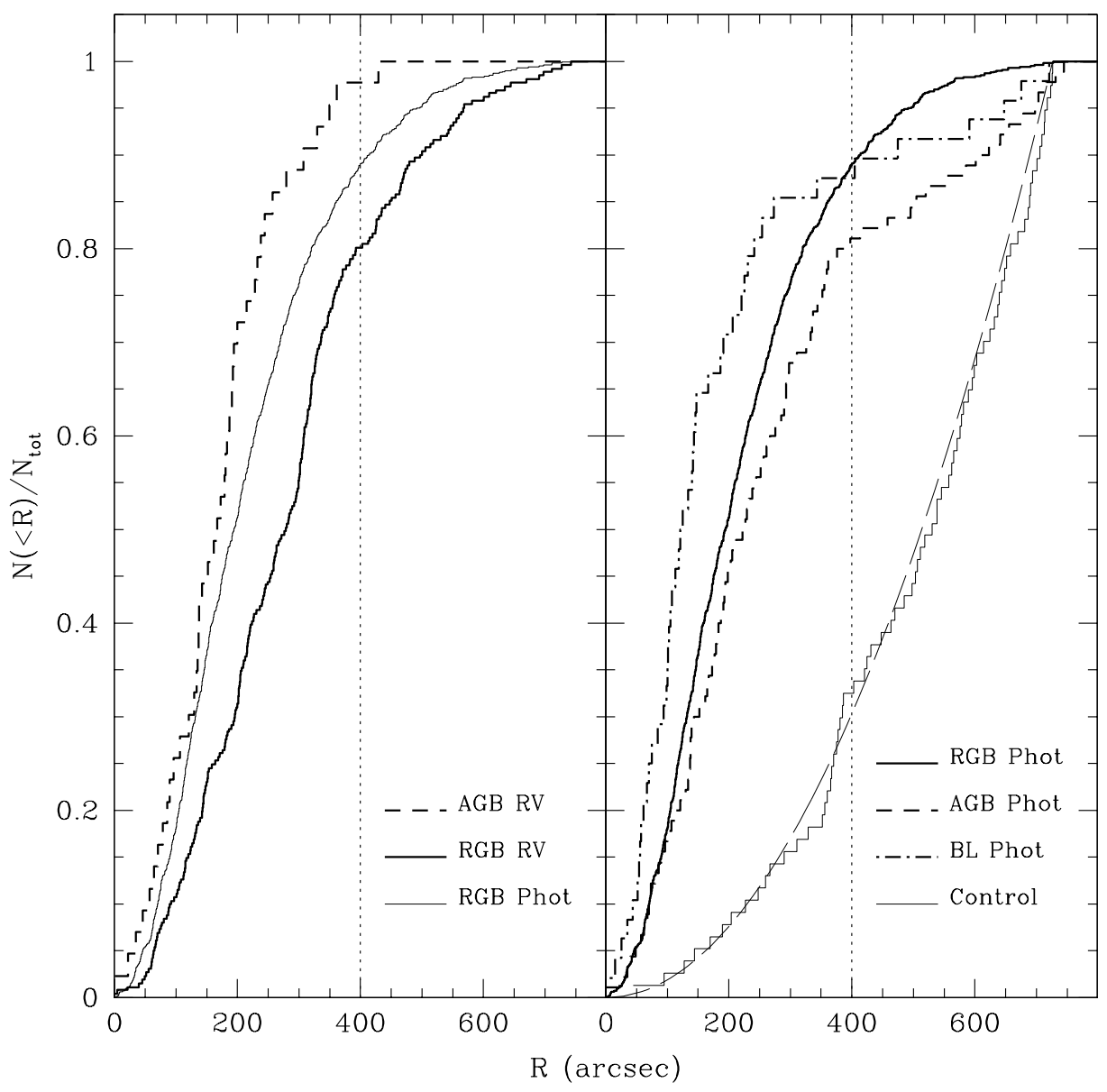

Fig. 24. - Left panel: The cumulative radial distributions of kinematically-confirmed member RGB (Region 1 of Figure 23, thick solid line; $N_{\text {tot }}=261$ stars) and AGB (Region 2; thick dashed line; 43 stars) stars of Leo I. The thin solid line is the distribution of photometricallyselected RGB stars (from Region 1 of Figure 23, 823 stars). The location of the break radius, $R_{b}$, is denoted by the dotted vertical line at $R=400$ arcsec. Right Panel: The cumulative radial distributions of photometrically-selected RGB (Region 1; thick solid line; 823 stars), AGB (Region 2; thick dashed line; 90 stars), and blue-loop (Region 4; dot-dashed line; 48 stars) stars identified in the Leo I CMD (Figure 23). Some idea of the completeness of our counts is given by the cumulative radial distribution of photometric non-members (Region 3; thin solid line; 77 stars). This is compared to the parabola $f=N(<R) / N_{\text {tot }}=\left(R / R_{\max }\right)^{2}$ (thin, long-dashed line) expected of a constant surface density contaminating population. The good fit suggests we are counting stars to fairly uniform completeness at all radii in Leo I. The break radius at $R=400$ arcsec is denoted as the dotted vertical line. 


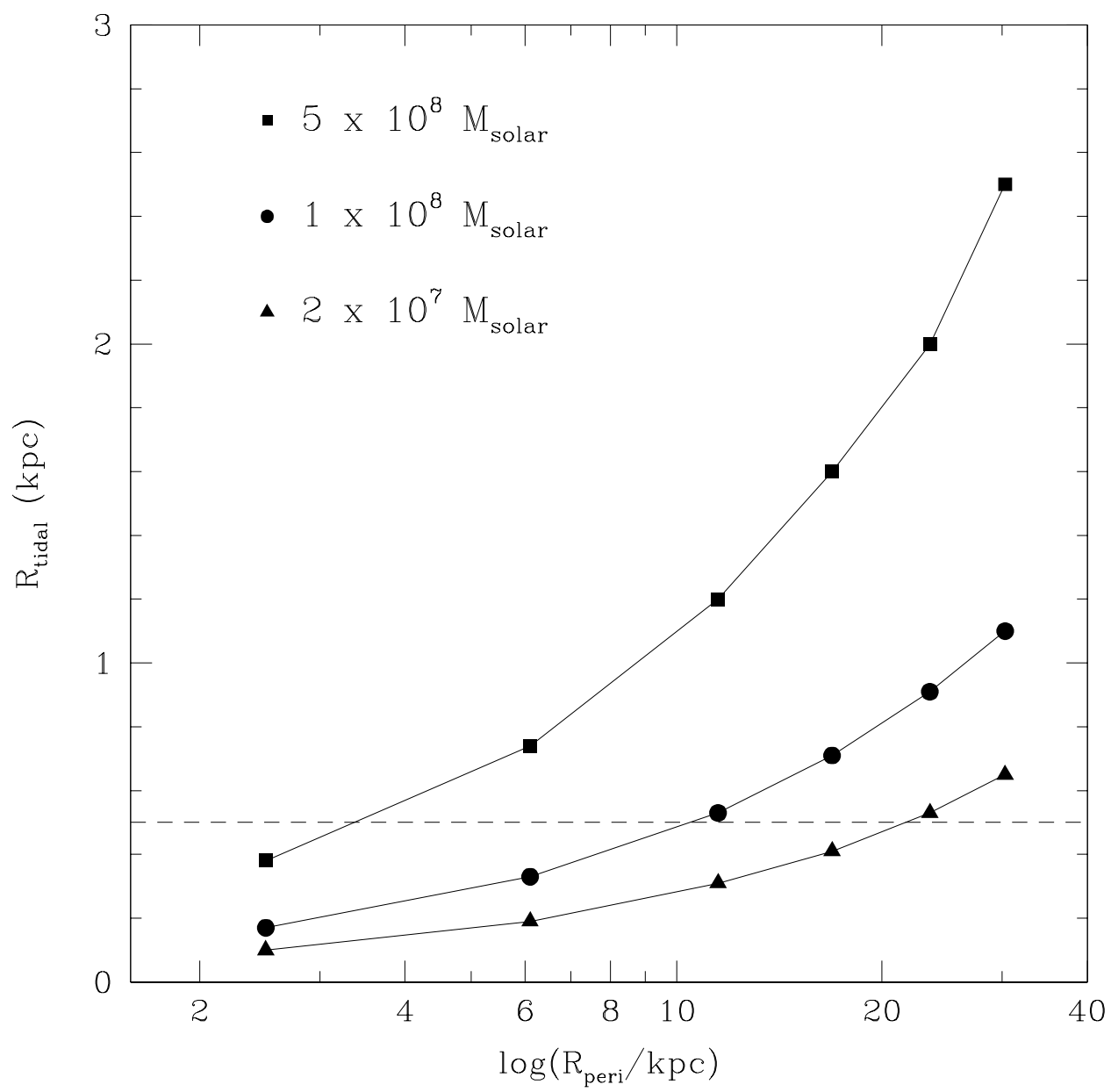

Fig. 25. - The instantaneous tidal radius $\left(R_{\text {tidal }}\right)$ of Leo I as a function of perigalactic distance for a range of assumed total masses (see Table 8 for more details of the orbits). The horizontal dashed line corresponds to the observed break radius $\left(R_{b} \sim 400 \operatorname{arcsec} \sim 500 \mathrm{pc}\right)$ at which the internal kinematics of Leo I begin to change from possible isotropy (interior to this radius) to streaming (exterior). A representative orbit is shown in Figure 13. 


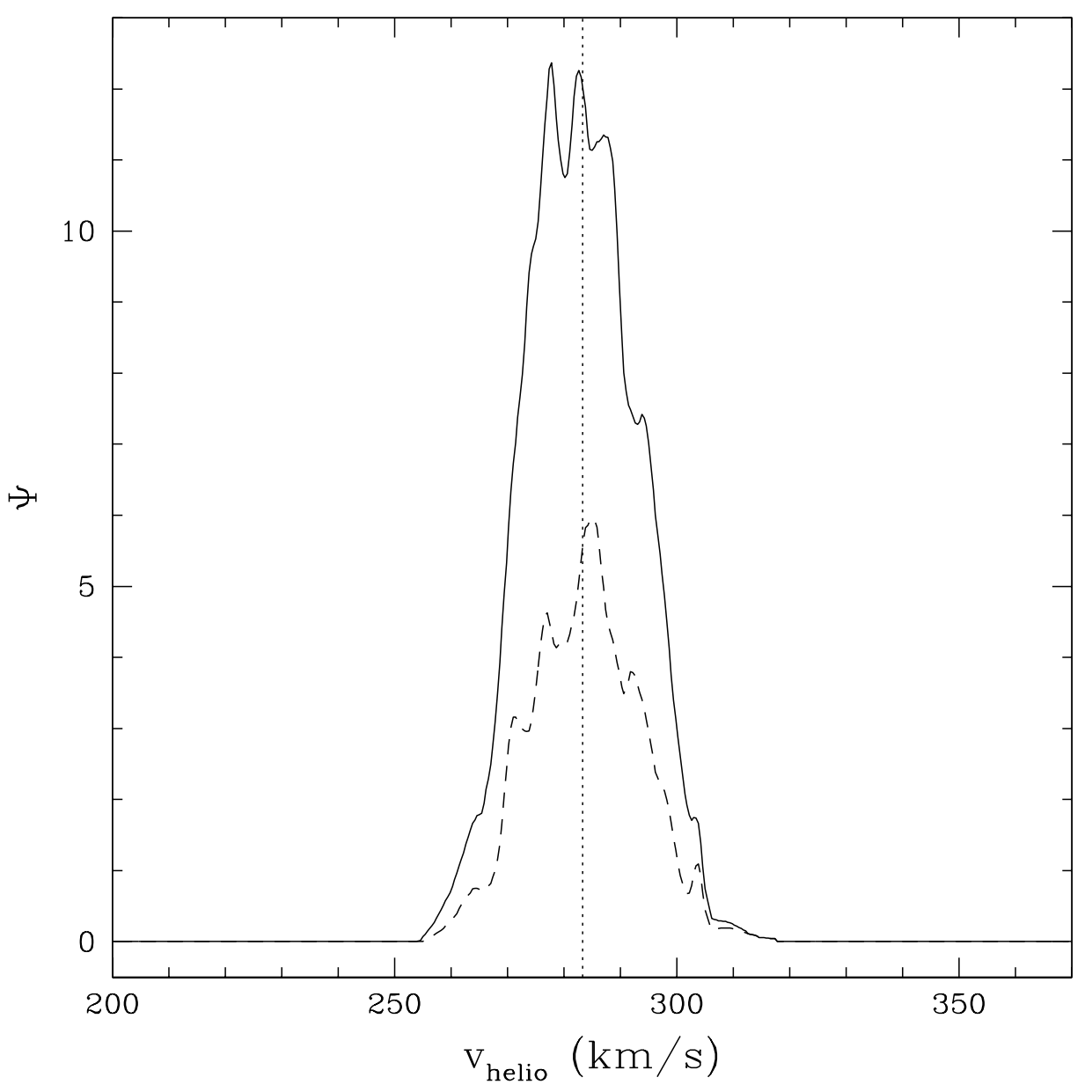

Fig. 26. - The velocity histogram of Leo I members. Each star is represented here as a normalized Gaussian centered at the star's heliocentric velocity and with $\sigma$ equal to the observed 1- $\sigma$ errors listed in Table 5 . The function $\Psi$ plotted here is the sum of the individual Gaussians sampled every $0.4 \mathrm{~km} / \mathrm{s}$. The vertical dashed line shows the mean heliocentric velocity of Leo I $(282.9 \mathrm{~km} / \mathrm{s}$; Section 4.3.1). The skew of this distribution is $0.08 \pm 0.14$ and the kurtosis is $-0.34 \pm 0.27$ (for $N=328$ ), both consistent with a Gaussian distribution. The dashed histogram is for stars from our kinematic sample selected from the regions on the sky observed by S07 (138 stars). We see no significant skew (0.10 \pm 0.21$)$, kurtosis $(-0.23 \pm 0.41)$, or velocity shift $(\langle v\rangle=283.7 \pm 0.7 \mathrm{~km} / \mathrm{s})$ for this subsample. These histograms are for heliocentric velocities, but, apart from a shift by $\sim-108 \mathrm{~km} / \mathrm{s}$, they would be essentially identical had we used Galactostationary velocities. 